\title{
Monoids of moduli spaces of manifolds
}

\author{
SøREn Galatius \\ OSCAR RANDAL-WILLIAMS
}

\begin{abstract}
We study categories of $d$-dimensional cobordisms from the perspective of Tillmann [14] and Galatius, Madsen, Tillman and Weiss [6]. There is a category $\mathcal{C}_{\theta}$ of closed smooth $(d-1)$-manifolds and smooth $d$-dimensional cobordisms, equipped with generalised orientations specified by a map $\theta: \mathbf{X} \rightarrow B O(d)$. The main result of [6] is a determination of the homotopy type of the classifying space $B \mathcal{C}_{\theta}$. The goal of the present paper is a systematic investigation of subcategories $\mathcal{D} \subseteq \mathcal{C}_{\theta}$ with the property that $B \mathcal{D} \simeq B \mathcal{C}_{\theta}$, the smaller such $\mathcal{D}$ the better.

We prove that in most cases of interest, $\mathcal{D}$ can be chosen to be a homotopy commutative monoid. As a consequence we prove that the stable cohomology of many moduli spaces of surfaces with $\theta$-structure is the cohomology of the infinite loop space of a certain Thom spectrum MT $\theta$. This was known for certain special $\theta$, using homological stability results; our work is independent of such results and covers many more cases.
\end{abstract}

57R90, 57R15, 57R56, 55P47

\section{Introduction and statement of results}

To state our results, we first recall from Galatius, Madsen, Tillman and Weiss [6] the definition of the $d$-dimensional cobordism category. Let us give the definition in outline here, and in full detail in Section 3.2. A tangential structure is a map $\theta: \mathbf{X} \rightarrow B O(d)$, and we write $\gamma \rightarrow B O(d)$ for the canonical vector bundle. A $\theta$-structure on a vector bundle is a bundle map (ie fibrewise linear isomorphism) from the vector bundle to $\theta^{*} \gamma$, and a $\theta$-structure on a manifold is a $\theta$-structure on its tangent bundle.

The objects of the cobordism category $\mathcal{C}_{\theta}$ are pairs $(M, l)$, where $M \subseteq \mathbb{R}^{\infty}$ is a closed $(d-1)$-manifold and $l$ is a $\theta$-structure on $\varepsilon^{1} \oplus T M$. The nonidentity morphisms of $\mathcal{C}_{\theta},\left(M_{0}, l_{0}\right) \rightarrow\left(M_{1}, l_{1}\right)$, are pairs $(t, W)$ with $t>0$ and $W \subseteq[0, t] \times \mathbb{R}^{\infty}$ an embedded cobordism, together with a $\theta$-structure $l$ on $W$ that agrees with $l_{0}$ and $l_{1}$ over the boundary. There is a "collar" condition on $(W, l)$ near $\partial W$, and the space of 
all such $W$ has a nice topology, described in detail in Section 3.2 (and also [6]). The homotopy type of morphism spaces is given by

$$
\mathcal{C}_{\theta}\left(M_{0}, M_{1}\right) \simeq \coprod_{W} B \operatorname{Diff}_{\theta}(W, \partial W),
$$

where $W$ ranges over connected compact cobordisms from $M_{0}$ to $M_{1}$, one in each diffeomorphism class rel $\partial W$. Diff $(W, \partial W)$ is the topological group of diffeomorphisms of $W$ which restrict to the identity on a neighbourhood of $\partial W$, and $B \operatorname{Diff}_{\theta}(W, \partial W)$ denotes the homotopy quotient

$$
B \operatorname{Diff}_{\theta}(W, \partial W)=\operatorname{Bun}^{\partial}\left(T W, \theta^{*} \gamma\right) / / \operatorname{Diff}(W, \partial W),
$$

where $\operatorname{Bun}^{\partial}\left(T W, \theta^{*} \gamma\right)$ is the space of bundle maps fixed near the boundary. We will say that $B \operatorname{Diff}_{\theta}(W, \partial W)$ is the moduli space of $d$-manifolds with $\theta$-structure, with underlying manifold diffeomorphic to $W$.

Finally, we recall the main theorem of [6] (reproved below as Theorem 3.12, largely following Chapter 6 of Galatius [5]). It determines the homotopy type of the classifying space of the cobordism category,

$$
B \mathcal{C}_{\theta} \simeq \Omega^{\infty-1} \mathbf{M T} \theta
$$

where $\mathbf{M T} \theta=\mathbf{X}^{-\theta}$ is the Thom spectrum of the inverse of the vector bundle classified by $\theta: \mathbf{X} \rightarrow B O(d)$.

To state our results, let us consider a version of $\mathcal{C}_{\theta}$ where manifolds have "basepoints". Pick once and for all a $\theta$-structure on the vector space $\mathbb{R}^{d}$. This induces a $\theta$-structure on any framed manifold, which we shall call the standard $\theta$-structure on that manifold.

Definition 1.1 Let $d \geq 2$. Let $\mathcal{C}_{\theta}^{\bullet}$ be the subcategory of $\mathcal{C}_{\theta}$ where objects $M \subseteq \mathbb{R}^{\infty}$ are required to contain the interval $(-\varepsilon, \varepsilon)^{d-1} \times\{0\} \subseteq \mathbb{R}^{\infty}$ for some $\varepsilon>0$ and to be connected. Morphisms $W \subseteq[0, t] \times \mathbb{R}^{\infty}$ are required to be connected and to contain the strip $[0, t] \times(-\varepsilon, \varepsilon)^{d-1} \times\{0\}$ for some $\varepsilon>0$. Furthermore, the tangential structure $l: T W \rightarrow \theta^{*} \gamma$ is required to be standard on the strip $[0, t] \times(-\varepsilon, \varepsilon)^{d-1} \times\{0\}$.

Homotopically, the result of this condition is to replace (1-1) by

$$
\mathcal{C}_{\theta}^{\bullet}\left(M_{0}, M_{1}\right) \simeq \coprod_{W} B \operatorname{Diff}_{\theta}(W, L \cup \partial W),
$$

where $W$ ranges over connected compact cobordisms between connected manifolds $M_{0}$ and $M_{1}$, containing an embedded arc $L$ connecting the two boundary components, one such $W$ in each diffeomorphism class relative to $\partial W \cup L$. 
Tangential structures and diffeomorphisms are required to be fixed near the line $L \subseteq W$. Thus we can cut open the surface $W$ along $L$, so the spaces in (1-3) are effectively moduli spaces of surfaces with one boundary component.

Theorem $\mathbf{A}$ Let $d \geq 2$ and $\theta: \mathbf{X} \rightarrow B O(d)$ be a tangential structure such that $\mathbf{X}$ is path connected and $S^{d}$ admits a $\theta-$ structure. Then the inclusion

$$
B \mathcal{C}_{\theta}^{\bullet} \rightarrow B \mathcal{C}_{\theta} \simeq \Omega^{\infty-1} \mathbf{M T} \theta
$$

is a weak homotopy equivalence.

Our main result is the following. Let us say that two objects in $\mathcal{C}_{\theta}^{\bullet}$ are cobordant if they give the same element in $\pi_{0} B C_{\theta}^{\bullet}=\pi_{-1} \mathbf{M T} \theta$. In Section 4.3 we show that this happens precisely when there is a morphism between the objects (not just a zig-zag).

Theorem B Let $\theta: \mathbf{X} \rightarrow B O(2)$ be a tangential structure such that $\mathbf{X}$ is path connected and that $S^{2}$ admits a $\theta$-structure. Let $\mathcal{D} \subseteq \mathcal{C}_{\theta}^{\bullet}$ be a full subcategory. Then the inclusion

$$
B \mathcal{D} \rightarrow B \mathcal{C}_{\theta}^{\bullet}
$$

is a weak homotopy equivalence of each component of $B \mathcal{D}$ onto a component of $B \mathcal{C}_{\theta}^{\bullet}$. If $\mathcal{D}$ has exactly one object, then it is a homotopy commutative monoid; if it has at most one object in each cobordism class, then it is a disjoint union of homotopy commutative monoids.

The assumption that $\mathbf{X}$ be path connected is innocent, since a disconnected $\mathbf{X}$ could be considered one path component at a time. The assumption that $S^{2}$ admits a $\theta$-structure is necessary for our proof, which uses surgery techniques. Under that assumption, the connected sum of two surfaces with $\theta$-structure will again have a $\theta$-structure (in contrast, the connected sum of eg framed surfaces is not framable; this corresponds to $\mathbf{X}=E O(2))$.

Let us spell out our result explicitly in the case of ordinary orientations (although it is not new in this case). Then $W$ in (1-3) ranges over connected oriented surfaces, and $B \operatorname{Diff}_{\theta}(W, \partial W \cup L)$ is homotopy equivalent to $B \Gamma_{g, 1}$, where $\Gamma_{g, 1}$ is the mapping class group of an oriented genus $g$ surface with one boundary component. The monoid $\mathcal{D}$ is homotopy equivalent to the disjoint union

$$
\mathcal{D} \simeq \coprod_{g \geq 0} B \Gamma_{g, 1},
$$

and the composition is the "pair of pants" composition of Miller [12]. Our result then says that this monoid has the same classifying spaces as the full cobordism category of [6]. Our proof is entirely geometric and does not rely on Harer stability. 


\subsection{Stabilisation and the Madsen-Weiss theorem}

Let us explain an application of Theorem B that highlights the advantage of homotopy commutativity. First we briefly discuss group completion of a homotopy commutative monoid $\mathcal{M}$, following McDuff and Segal [11]. There is a canonical map $\mathcal{M} \rightarrow \Omega B \mathcal{M}$ which is a homotopy equivalence if and only if $\mathcal{M}$ is grouplike, ie $\pi_{0} \mathcal{M}$ is a group. There is an induced map in homology

$$
H_{*}(\mathcal{M}) \rightarrow H_{*}(\Omega B \mathcal{M})
$$

which sends the multiplicative subset $\pi_{0} \mathcal{M} \subseteq H_{*}(\mathcal{M})$ to units of $H_{*}(\Omega B \mathcal{M})$, so there is an induced map from the localisation

$$
H_{*}(\mathcal{M})\left[\pi_{0} \mathcal{M}^{-1}\right] \rightarrow H_{*}(\Omega B \mathcal{M}) .
$$

The main theorem about group completion for homotopy commutative monoids (see McDuff and Segal [11]) is that (1-4) is an isomorphism of rings.

In many cases of interest, $\pi_{0} \mathcal{M}$ is a finitely generated monoid, so the localisation (1-4) can be formed as a sequential direct limit

$$
H_{*}(\mathcal{M}) \rightarrow H_{*}(\mathcal{M}) \rightarrow H_{*}(\mathcal{M}) \rightarrow \cdots
$$

over multiplication by an element $m$ which is the product of a set of generators. In fact, $\pi_{0} \mathcal{M}$ need not be finitely generated, it is only necessary that $\pi_{0} \mathcal{M}$ may be group completed by inverting finitely many elements. This direct limit is the homology of the space $\mathcal{M}_{\infty}$ obtained as the mapping telescope of the analogous direct system of spaces $\mathcal{M} \rightarrow \mathcal{M} \rightarrow \mathcal{M} \rightarrow \cdots$. Consequently we get a map $\mathcal{M}_{\infty} \rightarrow \Omega B \mathcal{M}$, inducing an isomorphism

$$
H_{*}\left(\mathcal{M}_{\infty}\right) \rightarrow H_{*}(\Omega B \mathcal{M}) .
$$

If $k$ is a field, the isomorphism (1-4) can be reinterpreted in terms of invariants of the action of $\pi_{0} \mathcal{M}$ on $H^{*}(\mathcal{M} ; k)$, namely that

$$
H^{*}(\mathcal{M} ; k)^{\pi_{0} \mathcal{M}} \cong H^{*}\left(\Omega_{0} B \mathcal{M} ; k\right),
$$

where $\Omega_{0}$ means the basepoint component of the loop space. Here the monoid $\pi_{0} \mathcal{M}$ acts on homology and cohomology of both spaces by translation. To deduce (1-5) we take coinvariants of the isomorphism (1-4) in $k$-homology and algebraically dualise, and note that the invariants of the action on $H^{*}(\Omega B \mathcal{M} ; k)$ is isomorphic to the cohomology of $\Omega_{0} B \mathcal{M}$.

We now apply this to the homotopy commutative monoid $\mathcal{D}$ produced by Theorem $\mathrm{B}$. The assumption that $\pi_{0} \mathcal{D}$ may be group completed by inverting finitely many elements holds in many cases of interest. The homology of a component of $\mathcal{D}_{\infty}$ can be interpreted 
as the homology of $B \operatorname{Diff}_{\theta}\left(W_{\infty}\right)$, the moduli space of infinite genus $\theta$-surfaces with parametrised germ at infinity.

The cohomology $H^{*}(\mathcal{D})$ is the ring of characteristic classes of bundles of $\theta$-surfaces with one parametrised boundary component. Then $H^{*}\left(\Omega_{0}^{\infty} \mathbf{M T} \theta\right)$ is the ring of characteristic classes that are invariant under gluing a trivial bundle onto the boundary.

Ebert [4] has recently proved that a similar result cannot hold for odd dimensional manifolds. He shows there are nontrivial rational classes in $H^{*}\left(\Omega^{\infty} \mathbf{M T S O}(2 n+1) ; \mathbb{Q}\right)$ which vanish in $H^{*}\left(B \operatorname{Diff}^{+}(M) ; \mathbb{Q}\right)$ for all oriented $(2 n+1)$-manifolds $M$.

We now give some particular cases of interest. Returning to the case of ordinary orientations, we reproduce the Madsen-Weiss theorem [10], that there is a homology equivalence

$$
\mathbb{Z} \times B \Gamma_{\infty, 1} \rightarrow \Omega^{\infty} \operatorname{MTSO}(2),
$$

where $\Gamma_{\infty, 1}$ is the limit of the mapping class groups $\Gamma_{g, 1}$ as $g \rightarrow \infty$.

Again we point out that we prove this result without quoting the homological stability results of Harer [7] and Ivanov [9]. The case of unoriented and spin surfaces can be treated similarly; cf Section 5.

For an oriented surface $F$, Cohen and Madsen [2] studied spaces

$$
\mathcal{S}_{g, n}(Y)=\operatorname{Map}^{\partial}\left(F_{g, n}, Y\right) / / \operatorname{Diff}^{+}\left(F_{g, n}\right)
$$

where $\mathrm{Map}^{\partial}$ is the space of maps taking $\partial F$ to the basepoint of $Y$, and Diff ${ }^{+}$denotes diffeomorphisms that preserve orientation and boundary. These are morphism spaces in the category $\mathcal{C}_{\theta}$, for $\theta: B \mathrm{SO}(2) \times Y \rightarrow B O(2)$, and it follows from [2] that, if $Y$ is simply connected, there is a homology equivalence

$$
\mathbb{Z} \times \mathcal{S}_{\infty, 1}(Y) \rightarrow \Omega^{\infty} \operatorname{MTSO}(2) \wedge Y_{+} .
$$

Here, $\pi_{0}(\mathcal{D})=\mathbb{N} \times H_{2}(Y ; \mathbb{Z})$ and $\mathbb{Z} \times \mathcal{S}_{\infty, 1}(Y)$ is the direct limit $\mathcal{D} \rightarrow \mathcal{D} \rightarrow \cdots$ over multiplication by an element corresponding to $(1,0) \in \mathbb{N} \times H_{2}(Y, \mathbb{Z})$. Our result provides an analogue to this for all connected spaces $Y$ and also to surfaces with any tangential structure satisfying the assumption of Theorem B, such as unoriented or spin surfaces.

The special case $Y=B \mathbb{Z}=S^{1}$ can be interpreted in complete analogy with (1-6). Let $v \in H^{1}\left(F_{g, 1} ; \mathbb{Z}\right)$ be a "primitive" cohomology class, ie one that can be extended to a symplectic basis of $H^{1}$ (equivalently, the Poincaré dual to an embedded nonseparating curve). Let $\Gamma_{g, 1}^{\prime} \leq \Gamma_{g, 1}$ denote the stabilizer of $v$. Then our results give a homology isomorphism

$$
\mathbb{Z} \times B \Gamma_{\infty, 1}^{\prime} \rightarrow \Omega^{\infty} \operatorname{MTSO}(2) \wedge S_{+}^{1}
$$


Similarly the case $X=B \mathbb{Z} / n$ gives a homology equivalence

$$
\mathbb{Z} \times B \Gamma_{\infty, 1}^{\prime}(n) \rightarrow \Omega^{\infty} \operatorname{MTSO}(2) \wedge B \mathbb{Z} / n_{+},
$$

where $\Gamma_{g, 1}^{\prime}(n) \leq \Gamma_{g, 1}$ denotes the subgroup that stabilizes the $\bmod n$ reduction of the primitive vector $v$. The space $B \Gamma_{g, 1}^{\prime}(n)$ has the same homotopy type as the moduli space of pairs $(\bar{\Sigma}, \Sigma)$ where $\Sigma$ is a genus $g$ Riemann surface with one boundary component and $\bar{\Sigma} \rightarrow \Sigma$ is an unbranched $n$-fold cyclic Galois cover.

For a completely general space $Y$ the monoid $\pi_{0} \mathcal{D}$ is difficult to understand explicitly, and our result must be stated as an algebraic isomorphism $H_{*}(\mathcal{D})\left[\pi_{0} \mathcal{D}^{-1}\right] \simeq$ $H_{*}\left(\Omega^{\infty} \mathbf{M T S O}(2) \wedge Y_{+}\right)$.

Acknowledgements S Galatius was partially supported by NSF grant DMS-0805843 and the Clay Mathematics Institute. O Randal-Williams was supported by an EPSRC Studentship, DTA grant number EP/P502667/1.

\section{Spaces of manifolds}

\subsection{Definitions}

Definition 2.1 Let $U \subseteq \mathbb{R}^{N}$ be an open set. Let $\Psi_{d}(U)$ be the set of subsets $M \subseteq U$ which are smooth $d$-dimensional submanifolds without boundary, and such that $M$ is closed as a subset. When the dimension is understood, we may simply write $\Psi(U)$.

If $V \subseteq U$, there is a restriction map $\Psi(U) \rightarrow \Psi(V)$ given by $M \mapsto M \cap V$. This makes $\Psi(U)$ into a sheaf of sets. We now define three topologies on $\Psi(U)$. The first two are used only as intermediate steps for defining the third. In Theorem 2.9 below we prove that the third topology makes $\Psi(-)$ into a sheaf of topological spaces.

Step 1 We first define the compactly supported topology. We will write $\Psi(U)^{\mathrm{cs}}$ for $\Psi(U)$ equipped with this topology. In fact, $\Psi(U)^{\mathrm{cs}}$ will be an infinite-dimensional smooth manifold, in which a neighbourhood of $M \in \Psi(U)^{\mathrm{cs}}$ is homeomorphic to a neighbourhood of the zero-section in the vector space $\Gamma_{c}(N M)$ consisting of compactly supported sections of the normal bundle $N M$ of $M \subseteq U$.

Let $C_{c}^{\infty}(M)$ denote the set of compactly supported smooth functions on $M$. Given a function $\varepsilon: M \rightarrow(0, \infty)$ and finitely many vector fields $X=\left(X_{1}, \ldots, X_{r}\right)$ on $M$ let $B(\varepsilon, X)$ denote the set of functions such that $\left|\left(X_{1} X_{2} \ldots X_{r} f\right)(x)\right|<\varepsilon(x)$ for all $x$. Declare the family of sets of the form $f+B(\varepsilon, X)$ a subbasis for the topology on $C_{c}^{\infty}(M)$, as $f$ ranges over $C_{c}^{\infty}(M), \varepsilon$ over functions $M \rightarrow(0, \infty)$, and $X$ over 
$r$-tuples of vector fields, and $r$ over nonnegative integers. This makes $C_{c}^{\infty}(M)$ into a locally convex vector space.

We define the normal bundle $N M$ to be the subbundle of $\varepsilon^{n}$ which is the orthogonal complement to the tangent bundle $T M \subseteq \varepsilon^{n}$. This identifies $\Gamma_{c}(N M)$ with a linear subspace of $C_{c}^{\infty}(M)^{\oplus n}$. We topologise it as a subspace.

By the tubular neighbourhood theorem, the standard map $N M \rightarrow \mathbb{R}^{n}$ restricts to an embedding of a neighbourhood of the zero section. Associating to a section $s$ its image $s(M)$ gives a partially defined injective map

$$
\Gamma_{c}(N M) \stackrel{c_{M}}{-\rightarrow} \Psi(U)^{\mathrm{cs}}
$$

whose domain is an open set. Topologise $\Psi(U)^{\text {cs }}$ by declaring the maps $c_{M}$ to be homeomorphisms onto open sets. This makes $\Psi(U)^{\mathrm{cs}}$ into an infinite dimensional manifold, modelled on the topological vector spaces $\Gamma_{c}(N M)$.

Step 2 For each compact set $K$, we define a topology on $\Psi(U)$, called the $K-$ topology. We will write $\Psi(U)^{K}$ for $\Psi(U)$ equipped with this topology.

Let

$$
\Psi(U)^{\mathrm{cs}} \stackrel{\pi_{K}}{\longrightarrow} \Psi(K \subseteq U)
$$

be the quotient map that identifies elements of $\Psi(U)^{\mathrm{cs}}$ if they agree on a neighbourhood of $K$. The image of a manifold $M \in \Psi(U)^{\text {cs }}$ is the germ of $M$ near $K$, and we shall also write $\pi_{K}(M)=\left.M\right|_{K}$. Give $\Psi(K \subseteq U)$ the quotient topology.

Now, let $\Psi(U)^{K}$ be the topological space with the same underlying set as $\Psi(U)^{\text {cs }}$, and with the coarsest topology making $\pi_{K}: \Psi(U)^{K} \rightarrow \Psi(K \subseteq U)$ continuous. It is a formal consequence of the universal properties of initial and quotient topologies that the identity map $\Psi(U)^{L} \rightarrow \Psi(U)^{K}$ is continuous when $K \subseteq L$ are two compact sets. That is, the $L$-topology is finer than the $K$-topology.

Step 3 Finally, let $\Psi(U)$ have the coarsest topology finer than all the $K$-topologies. In other words, $\Psi(U)$ is the inverse limit of $\Psi(U)^{K}$ over larger and larger compact sets.

Example 2.2 The simplest example is taking $\{t\} \times \mathbb{R} \subseteq \mathbb{R}^{2}$ as a function of $t$. This a path in $\Psi_{1}\left(\mathbb{R}^{2}\right)$, and as $t \rightarrow \infty$ it converges to the empty manifold $\varnothing$. This is because for each compact subset $K \subseteq \mathbb{R}^{2}, K \cap\{t\} \times \mathbb{R}$ is empty for all sufficiently large $t$, so it converges to $\varnothing$ in the $K$-topology, for all $K$. 


\subsection{Elementary properties and constructions}

Let $V \subseteq U$. The restriction map $\Psi(U)^{\mathrm{cs}} \rightarrow \Psi(V)^{\mathrm{cs}}$ is not continuous. We have the following result instead.

Lemma 2.3 The restriction map $r: \Psi(U)^{\mathrm{cs}} \rightarrow \Psi(V)^{\mathrm{cs}}$ is an open map.

Proof Let $M \in \Psi(U)^{\mathrm{cs}}$. We have the commutative diagram

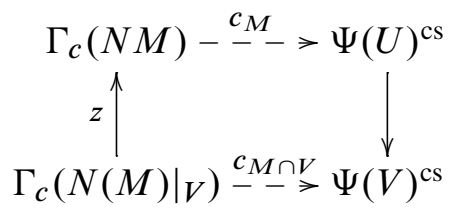

where $z$ denotes extension by 0 , which is continuous. This induces a partially defined right inverse $\Psi(V)^{\mathrm{cs}} \rightarrow \Psi(U)^{\mathrm{cs}}$ to the restriction map. The right inverse is defined in an open neighbourhood of every point in the image of the restriction; this proves the claim.

The following technical result will be used several times.

Lemma 2.4 Let $K \subseteq U$ be compact. Let $0<3 \varepsilon \leq \operatorname{dist}\left(K, \mathbb{R}^{n}-U\right)$ and let $\lambda: \mathbb{R}^{n} \rightarrow[0,1]$ be a smooth function such that $\lambda(x)=1$ if $\operatorname{dist}(x, K) \leq \varepsilon$ and $\lambda(x)=0$ if $\operatorname{dist}(x, K) \geq 2 \varepsilon$. If the support of $\lambda$ is contained in an open set $V \subseteq U$, then multiplication by $\lambda$ gives a (continuous!) map $\bar{\lambda}: \Gamma_{c}(N M) \rightarrow \Gamma_{c}(N(M \cap V))$. If we let $z: \Gamma_{c}(N(M \cap V)) \rightarrow \Gamma_{c}(N M)$ denote extension by zero, we have the diagram

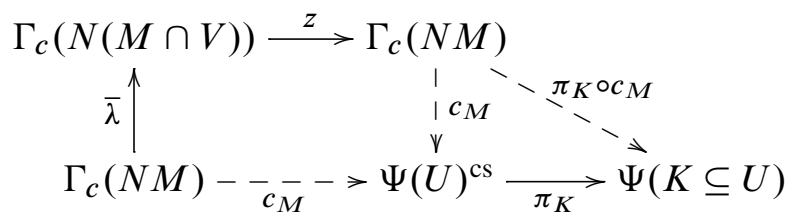

in which the triangle and the outer pentagon commute after possibly restricting to a smaller neighbourhood of $0 \in \Gamma_{c}(N M)$

Proof The pentagon commutes for sections $h \in \Gamma_{c}(N M)$ satisfying $|h(x)|<\varepsilon$, and this is an open condition.

Lemma 2.5 The quotient map $\pi_{K}: \Psi(U)^{\mathrm{cs}} \rightarrow \Psi(K \subseteq U)$ is an open map. 
Proof We are claiming that $\left(\pi_{K}\right)^{-1}\left(\pi_{K}(A)\right)$ is open for all open $A \subseteq \Psi(U)^{\mathrm{cs}}$. Let $M \in\left(\pi_{K}\right)^{-1}\left(\pi_{K}(A)\right)$. This means that $M \cap V=M^{\prime} \cap V$ for some open $V \supseteq K$ and some $M^{\prime} \in A$. Now the composition $\pi_{K} \circ c_{M}: \Gamma_{c}(N M) \rightarrow \Psi(U)^{\mathrm{cs}} \rightarrow \Psi(K \subseteq U)$ can be factored as in Lemma 2.4. Thus, if we want to check that some point $M \in$ $\left(\pi_{K}\right)^{-1}\left(\pi_{K}(A)\right)$ is interior, it suffices to check that the inverse image of $\pi_{K}(M)$ in $\Gamma_{c}(N(M \cap V))$ is a neighbourhood of 0 .

This holds for $M^{\prime} \in A \subseteq\left(\pi_{K}\right)^{-1}\left(\pi_{K}(M)\right)$, and hence also holds for $M$ since they agree inside $V$.

Lemma 2.6 Let $V \subseteq U$. The injection $\rho: \Psi(K \subseteq U) \rightarrow \Psi(K \subseteq V)$ is a homeomorphism onto an open subset.

Proof Continuity follows from Lemma 2.4. Indeed, we get a diagram

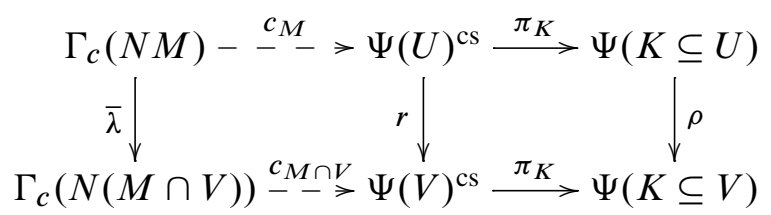

where the outer rectangle and right hand square commute. This proves that $\rho \circ \pi_{K} \circ c_{M}$ is continuous. Since $\pi_{K}$ is a quotient map and $c_{M}$ is a local homeomorphism, this proves that $\rho$ is continuous.

To see that $\rho(A)$ is open when $A \subseteq \Psi(K \subseteq U)$ is open, let $B=\left(\pi_{K}\right)^{-1}(A)$. Then $\rho(A)=\pi_{K} \circ r(B)$. Since $\pi_{K}$ and $r$ are both open maps, by Lemmas 2.5 and 2.3, $\rho(A)$ is open.

Theorem 2.7 For $V \subseteq U$, the restriction map $\Psi(U) \rightarrow \Psi(V)$ is continuous.

Proof We need to show that the composition $\Psi(U) \rightarrow \Psi(V) \rightarrow \Psi(K \subseteq V)$ is continuous for all compact $K \subseteq V$. This follows from Lemma 2.6.

Lemma 2.8 Let $K_{i} \subseteq U$ be compact, $i=1, \ldots, r$, and let $K=\bigcup_{i} K_{i}$. Then the diagonal map $\delta: \Psi(U)^{K} \rightarrow \prod \Psi(U)^{K_{i}}$ is a homeomorphism onto its image.

Proof Continuity of $\delta$ follows from continuity of each $\Psi(U)^{K} \rightarrow \Psi(U)^{K_{i}}$. Let $\Delta=\delta\left(\Psi(U)^{K}\right)$. We need to see that $\delta: \Psi(U)^{K} \rightarrow \Delta$ is open. We have a diagram

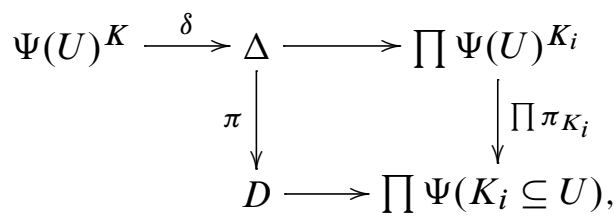


where $D=\left(\prod \pi_{K_{i}}\right)(\Delta)$, the horizontal maps are inclusions of subsets, and $\pi$ is the restriction of $\prod \pi_{K_{i}}$. A set-theoretic consideration shows that for any subset $A \subseteq \Psi(U)$ we have

$$
\pi^{-1} \pi \delta(A)=\left(\prod \pi_{K_{i}}^{-1} \pi_{K_{i}}(A)\right) \cap \Delta .
$$

Now consider the diagram

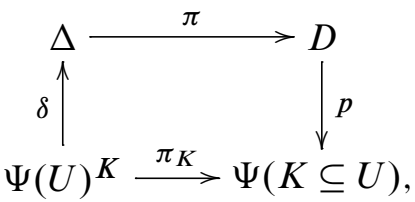

where $p$ is defined by commutativity of the diagram: $p(\pi \delta(M))=\pi_{K}(M)$. This is well defined because both $\pi$ and $\delta$ are surjective, and because if $\pi \delta(M)=\pi \delta\left(M^{\prime}\right)$, then $M$ and $M^{\prime}$ agree in a neighbourhood of each $K_{i}$ and hence agree in a neighbourhood of $K$, so $\pi_{K}(M)=\pi_{K}\left(M^{\prime}\right)$. By Lemma 2.5, the open neighbourhoods of $M \in \Psi(U)^{K}$ are precisely of the form $\pi_{K}^{-1} \pi_{K}(A)$, for $M \in A \subseteq \Psi(U)^{\mathrm{cs}}$ open. We need to prove that the set

$$
\delta\left(\pi_{K}^{-1} \pi_{K}(A)\right) \subseteq \Delta
$$

is a neighbourhood of $\delta(M)$. By diagram (2-3), we can replace $\pi_{K}(A)$ by $p \pi \delta(A)$, and replace $\delta \pi_{K}^{-1}$ by $\pi^{-1} p^{-1}$. Using (2-2) this gives

$$
\delta\left(\pi_{K}^{-1} \pi_{K}(A)\right)=\pi^{-1} p^{-1} p \pi \delta(A) \supseteq \pi^{-1} \pi \delta(A)=\left(\prod \pi_{K_{i}}^{-1} \pi_{K_{i}}(A)\right) \cap \Delta,
$$

which is an open subset of $\Delta$, containing $\delta(M)$.

Theorem 2.9 Let $f: X \rightarrow \Psi(U)$ be a map such that each $x \in U$ has an open neighbourhood $U_{x} \subseteq U$ such that the composition $X \rightarrow \Psi(U) \rightarrow \Psi\left(U_{x}\right)$ is continuous. Then $X \rightarrow \Psi(U)$ is continuous.

Proof We need to show that $f: X \rightarrow \Psi(U)^{K}$ is continuous for any compact $K \subseteq U$. $K$ is covered by finitely many of the $U_{x}$ 's, say $U_{1}, \ldots, U_{r}$. Pick $K_{i} \subseteq U_{i}$ compact with $K \subseteq \cup K_{i}$. Then the composition

$$
X \stackrel{f}{\rightarrow} \Psi(U)^{K_{i}} \rightarrow \Psi\left(K_{i} \subseteq U\right) \rightarrow \Psi\left(K_{i} \subseteq U_{i}\right)
$$

is continuous for each $i$ by assumption. By Lemma 2.6, the composition $X \rightarrow \Psi(U) \rightarrow$ $\Psi\left(K_{i} \subseteq U\right)$ is continuous, and hence each $X \rightarrow \Psi(U)^{K_{i}}$ is continuous. It now follows from Lemma 2.8 that $X \rightarrow \Psi(U)^{K}$ is continuous as required. 
Lemma 2.10 Let $\operatorname{Emb}(U, V)$ denote the space of embeddings of one open subset of $\mathbb{R}^{N}$ into another, and let $j_{0} \in \operatorname{Emb}(U, V)$. Then there exists a partially defined map

$$
\operatorname{Emb}(U, V) \stackrel{\varphi}{\stackrel{-}{\rightarrow}} \operatorname{Diff}_{c}(U),
$$

defined in an open neighbourhood of $j_{0}$, such that $j(x)=j_{0} \circ(\varphi(j))(x)$ for $x$ in a neighbourhood of $K$.

Proof Pick a compactly supported function $\lambda: U \rightarrow[0,1]$ with $K \subseteq \operatorname{int}\left(\lambda^{-1}(0)\right)$. Then let

$$
(\varphi(j))(x)=(1-\lambda(x)) x+\lambda(x)\left(j_{0}^{-1} \circ j\right)(x),
$$

which defines a compactly supported diffeomorphism $U \rightarrow U$ for all $j$ sufficiently close to $j_{0}$.

Theorem 2.11 The map

$$
\operatorname{Emb}(U, V) \times \Psi(V) \rightarrow \Psi(U),
$$

given by $(j, M) \mapsto j^{-1}(M)$, is continuous.

Proof It suffices to see that the composition

$$
\operatorname{Emb}(U, V) \times \Psi(V) \rightarrow \Psi(U) \rightarrow \Psi(K \subseteq U)
$$

is continuous in a neighbourhood of each $\left\{j_{0}\right\} \times \Psi(V)$, for each $K \subseteq U$ compact. But this follows from the previous lemma, which implies that the map factors through $\operatorname{Diff}_{c}(U)$, which acts continuously on $\Psi(U)$.

\subsection{Tangential structures}

We will study analogues of the point-set topological results from the previous section, where all manifolds $M \in \Psi_{d}(U)$ are equipped with some tangential structure. Examples are orientations, spin structures, almost complex structures, etc.

Definition 2.12 Let $\theta: \mathbf{X} \rightarrow B O(d)$ be a map. A $\theta$-structure on $M \in \Psi_{d}(U)$ is a bundle map (ie fibrewise linear isomorphism) $l: T M \rightarrow \theta^{*} \gamma$. Let $\Psi_{\theta}(U)$ denote the set of pairs $(M, l)$, where $M \in \Psi_{d}(U)$ and $l$ is a $\theta$-structure on $M$.

More generally, a $\theta_{d-k}$-structure on $M \in \Psi_{d-k}(U)$ is a $\theta$-structure on $\varepsilon^{k} \oplus T M$. Let $\Psi_{\theta_{d-k}}(U)$ denote the set of pairs $(M, l)$, where $M \in \Psi_{d-k}(U)$ and $l$ is a $\theta_{d-k^{-}}$ structure on $M$. 
Often we will omit the tangential structure and just write $M \in \Psi_{\theta}\left(\mathbb{R}^{n}\right)$ instead of $(M, l) \in \Psi_{\theta}\left(\mathbb{R}^{n}\right)$. We can define a space $\mathbf{X}_{d-k}$ and a map $\theta_{d-k}$ by the pullback diagram

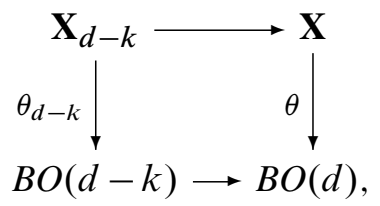

where $B O(d-k)$ is defined to be $\operatorname{Bun}\left(\mathbb{R}^{k} \times \mathbb{R}^{d-k} \rightarrow *, \gamma\right) / G L_{d-k}(\mathbb{R})$. With this definition of $\theta_{d-k}$, the two definitions of the set $\Psi_{\theta_{d-k}}(U)$ in Definition 2.12 are in natural bijection.

Remark 2.13 A map $\mathbf{X} \rightarrow \mathbf{X}^{\prime}$ over $B O(d)$ induces natural maps of all the spaces occurring in Theorems $\mathrm{A}$ and $\mathrm{B}$, and if $\mathbf{X} \rightarrow \mathbf{X}^{\prime}$ is a homotopy equivalence then so are these maps. Hence, we may assume that $\theta: \mathbf{X} \rightarrow B O(d)$ is a Serre fibration, which we will do from now on.

Clearly $\Psi_{\theta}$ is again a sheaf of sets. We wish to endow it with a topology so that it is a sheaf of topological spaces. We start by defining the analogue of the compactly supported topology. For $M \in \Psi(U)^{\mathrm{cs}}$, let $\operatorname{Bun}\left(T M, \theta^{*} \gamma\right)$ be the space of bundle maps, topologised in the compact-open topology, and let $\Gamma_{c}(N M) \times \operatorname{Bun}\left(T M, \theta^{*} \gamma\right)$ have the product topology. For $s \in \Gamma(N M)$ close to the zero section, we have $c_{M}(s)=s(M) \in \Psi(U)^{\mathrm{cs}}$. We also get the bundle isomorphism $D s: T M \rightarrow T\left(c_{M}(s)\right)$ and hence $(D s)^{-1} \circ l$ is a $\theta$-structure on $c_{M}(s)$. This gives a map

$$
c_{M}^{\theta}: \Gamma_{c}(N M) \times \operatorname{Bun}\left(T M, \theta^{*} \gamma\right) \rightarrow \Psi_{\theta}(U)^{\mathrm{cs}}
$$

$\operatorname{viz}(s, l) \mapsto\left(s(M),(D s)^{-1} \circ l\right)$. It is clear that $c_{M}^{\theta}$ is injective and that the image is $u^{-1}\left(\operatorname{Im}\left(c_{M}\right)\right)$, where $u: \Psi_{\theta}(U) \rightarrow \Psi(U)$ is the forgetful map $(M, l) \mapsto M$. Topologise $\Psi_{\theta}(U)^{\mathrm{cs}}$ by declaring the maps $c_{M}^{\theta}$ homeomorphisms onto open sets.

Then define $\Psi_{\theta}(K \subseteq U), \Psi_{\theta}(U)^{K}$ and $\Psi_{\theta}(U)$ from $\Psi_{\theta}(U)^{\mathrm{cs}}$ as in Steps 2 and 3 in Section 2.1. It is not hard to modify the proofs of Theorems 2.7, 2.9 and 2.11 to work also in the presence of $\theta$-structures. We summarise the result in the following theorem.

Theorem 2.14 $\Psi_{\theta}$ is a sheaf of topological spaces on $\mathbb{R}^{n}$. The map

$$
\operatorname{Emb}(U, V) \times \Psi_{\theta}(U) \rightarrow \Psi_{\theta}(V)
$$

is continuous. 
Proof sketch First we have, with the same proof, an analogue of Lemma 2.4 where the two $\Psi$ are replaced by $\Psi_{\theta}$ and all three spaces of sections of normal bundles in the diagram are replaced with their product with $\operatorname{Bun}\left(T M, \theta^{*} \gamma\right)$. Once that is established, the $\theta$ analogues of Theorem 2.7 and Theorem 2.9 are proved in the exact same way. This proves that $\Psi_{\theta}$ is a sheaf of spaces.

An embedding $j: U \rightarrow V$ gives a map $\Psi_{\theta}(U) \rightarrow \Psi_{\theta}(V)$ which sends $(M, l)$ to $\left(j^{-1} M, l \circ\left(\left.D j\right|_{j^{-1}(M)}\right)\right)$. This defines the map in the theorem, and its continuity is proved exactly as in Theorem 2.11 .

\subsection{Smooth maps}

In practice it can be tedious to check continuity of a map $X \rightarrow \Psi_{\theta}\left(\mathbb{R}^{n}\right)$. If $X$ is smooth there is an easier property to verify.

Definition 2.15 Let $f: X \rightarrow \Psi_{\theta}(U)$ be a continuous map, and write $f(x)=\left(M_{x}, l_{x}\right)$. Define the graph of $f$ to be the space of pairs

$$
\Gamma(f)=\bigcup_{x \in X}\{x\} \times M_{x} \subseteq X \times U
$$

and the vertical tangent bundle to be

$$
T^{v} \Gamma(f)=\bigcup_{x \in X}\{x\} \times T M_{x} \subseteq X \times T U,
$$

both with the subspace topology. The $l_{x}$ determine a bundle map $l(f): T^{v} \Gamma(f) \rightarrow$ $\theta^{*} \gamma$.

Definition 2.16 If $X$ is a manifold, say a continuous map $f: X \rightarrow \Psi_{\theta}\left(\mathbb{R}^{n}\right)$ is smooth if $\Gamma(f) \subseteq X \times U$ is a smooth submanifold and $\pi: \Gamma(f) \rightarrow X$ is a submersion.

More generally say it is smooth near $(x, u) \in X \times U$ if there are neighbourhoods $A \subseteq X$ of $x$ and $B \subseteq U$ of $u$ such that $A \rightarrow X \rightarrow \Psi_{\theta}(U) \rightarrow \Psi_{\theta}(B)$ is smooth. For a closed $C \subseteq X \times U$ say $f$ is smooth near $C$ if it is smooth near every point of $B$.

Lemma 2.17 If $X$ is a $k$-dimensional smooth manifold, there is a bijection between the set of smooth maps $f: X \rightarrow \Psi_{\theta}(U)$ and the set of pairs $(\Gamma, l)$ where $\Gamma \subseteq X \times U$ is a smooth $(d+k)$-dimensional submanifold that is closed as a subspace and such that $\pi_{X}: \Gamma \rightarrow X$ is a submersion, and $l: \operatorname{Ker}\left(D \pi_{X}: T \Gamma \rightarrow T X\right) \rightarrow \theta^{*} \gamma$ is a bundle map.

Proof A smooth map $f$ determines a graph $\Gamma(f)$ and a bundle map $l(f): T^{v} \Gamma(f) \rightarrow$ $\theta^{*} \gamma$ having the required properties. 
Given a pair $(\Gamma, l)$, there is certainly a map of sets $f: X \rightarrow \Psi_{\theta}(U)$ given by $x \mapsto$ $\left(\pi_{X}^{-1}(x),\left.l\right|_{\pi_{X}^{-1}(x)}: T \pi_{X}^{-1}(x) \rightarrow \theta^{*} \gamma\right)$. Then $\Gamma(f)=\Gamma, T^{v} \Gamma(f)=T^{v} \Gamma$ and $l(f)=l$. We must just check that this $f$ is continuous.

By the local submersion theorem, we can choose local coordinates $\mathbb{R}^{k} \rightarrow X$ and $\mathbb{R}^{n} \rightarrow U$ inside which $\Gamma \subseteq X \times U$ is the image of an embedding $\mathbb{R}^{d+k} \rightarrow \mathbb{R}^{k} \times \mathbb{R}^{n}$ such that $\mathbb{R}^{d+k} \rightarrow \mathbb{R}^{k}$ is the projection onto the first $k$ coordinates. This produces a continuous map $\mathbb{R}^{k} \rightarrow \operatorname{Emb}\left(\mathbb{R}^{d}, \mathbb{R}^{n}\right) \times \operatorname{Bun}\left(T \mathbb{R}^{d}, \theta^{*} \gamma\right)$, which is smooth on the first coordinate. We may suppose that the origin in $\mathbb{R}^{k}$ is sent to the standard embedding $e: \mathbb{R}^{d} \hookrightarrow \mathbb{R}^{n}$. There is a map, defined near $e$, from $\operatorname{Emb}\left(\mathbb{R}^{d}, \mathbb{R}^{n}\right)$ to $\operatorname{Emb}\left(\mathbb{R}^{d}, \mathbb{R}^{d}\right) \times C^{\infty}\left(\mathbb{R}^{d}, \mathbb{R}^{n-d}\right)$, and we identify the second factor with the space $\Gamma\left(N e\left(\mathbb{R}^{d}\right)\right)$. Let us write $B_{k}(r)$ for the ball of radius $r$ inside $\mathbb{R}^{k}$. There is also a map $(-)^{-1}: \operatorname{Emb}\left(\mathbb{R}^{d}, \mathbb{R}^{d}\right) \rightarrow \operatorname{Emb}\left(B_{d}(2), \mathbb{R}^{d}\right)$ given on a neighbourhood of the identity by $e \mapsto e^{-1}: B_{d}(2) \rightarrow \mathbb{R}^{d}$, as on a neighbourhood of the identity embeddings $e$ contain the ball $B_{d}(2)$ in their image.

Composing with the action via pullback gives a map

$$
\begin{aligned}
\operatorname{Emb}\left(\mathbb{R}^{d}, \mathbb{R}^{n}\right) & \rightarrow \operatorname{Emb}\left(\mathbb{R}^{d}, \mathbb{R}^{d}\right) \times \Gamma\left(N e\left(\mathbb{R}^{d}\right)\right) \\
& \rightarrow \operatorname{Emb}\left(B_{d}(2), \mathbb{R}^{d}\right) \times \Gamma\left(N e\left(\mathbb{R}^{d}\right)\right) \rightarrow \Gamma\left(N e\left(B_{d}(2)\right)\right)
\end{aligned}
$$

which sends an embedding $f$ to a section of $N e\left(B_{d}(2)\right)$ having the same image inside $B_{n}(1) \subseteq \mathbb{R}^{n}$.

Choosing a compactly supported function $\varphi: B_{d}(2) \rightarrow[0,1]$ that is identically 1 inside the unit ball and multiplying sections by it gives a continuous map to $\Gamma_{c}\left(N e\left(B_{d}(2)\right)\right)$, so we have a sequence of continuous maps

$$
\mathbb{R}^{k} \rightarrow \operatorname{Emb}\left(\mathbb{R}^{d}, \mathbb{R}^{n}\right)-\rightarrow \Gamma\left(N e\left(B_{d}(2)\right)\right) \stackrel{\cdot \varphi}{\longrightarrow} \Gamma_{c}\left(N e\left(B_{d}(2)\right)\right) .
$$

In total, we obtain a continuous map

$$
\mathbb{R}^{k} \rightarrow \Gamma_{c}\left(N e\left(B_{d}(2)\right)\right) \times \operatorname{Bun}\left(T B_{d}(2), \theta^{*} \gamma\right) \rightarrow \Psi_{\theta}\left(B_{n}(2)\right)
$$

defined near the origin, that agrees with $\mathbb{R}^{k} \rightarrow X \stackrel{f}{\rightarrow} \Psi_{\theta}(U) \rightarrow \Psi_{\theta}\left(\mathbb{R}^{n}\right)$ after restricting both to the unit ball $B_{n}(1) \subseteq \mathbb{R}^{n}$. In particular, by Theorem $2.9 f$ is continuous.

Lemma 2.18 Let $X$ be a smooth manifold and $f: X \rightarrow \Psi(U)$ be a continuous map. Let $V \subseteq X \times U$ be open, and $W \subseteq X \times U$ be such that $\bar{V} \subseteq \operatorname{int}(W)$. Then there exists a homotopy $F:[0,1] \times X \rightarrow \Psi(U)$ starting at $f$, which is smooth on $(0,1] \times V \subseteq[0,1] \times X \times U$ and is the constant homotopy outside $W$. Furthermore, if $f$ is already smooth on an open set $A \subseteq V$, then the homotopy can be assumed smooth on $[0,1] \times A$. 
Proof Let us say that the open set $W \subseteq X \times U$ is small if there are closed sets $K \subseteq X$, $L \subseteq U$, such that $W \subseteq K \times L$ and the composition

$$
K \rightarrow X \rightarrow \Psi(U) \rightarrow \Psi(L)
$$

factors through a continuous map $K \rightarrow \Psi(U)^{\mathrm{cs}}$. Then we can use the manifold structure on $\Psi(U)^{\text {cs }}$ to find a homotopy $[0,1] \times K \rightarrow \Psi(U)^{\text {cs }}$ which is smooth on $(0,1] \times K$. Using a suitable bump function we can ensure that it is the constant homotopy outside $W \subseteq K \times U$, and hence extends to a homotopy $[0,1] \times X \rightarrow \Psi(U)$ which is constant outside $W$.

Next we consider the case where $W$ is the disjoint union of small open sets. This is easy, since we can superimpose the corresponding homotopies. After restricting the homotopy to $[0, \varepsilon] \times X$ and composing with the linear diffeomorphism $[0,1] \rightarrow[0, \varepsilon]$, we can assume that the homotopy is arbitrarily small.

For general $\mathrm{W}$, we first triangulate $X \times U$ such that every simplex is contained in a small open set. For each $p$-simplex $\sigma \subseteq X \times U$, let $\operatorname{st}(\sigma) \subseteq X \times U$ be the open star of $\sigma$, thought of as a vertex of the barycentric subdivision. Let $W^{p} \subseteq X \times U$ be the union of $\operatorname{st}(\sigma)$ over all $p$-simplices $\sigma$. Then $X \times U$ is the (finite) union of the $W^{p}$. We can pick slightly smaller open sets $V^{p} \subseteq W^{p}$ such that $\overline{V^{p}} \subseteq \operatorname{int}\left(W^{p}\right)$ and such that the $V^{p}$ still cover $X \times U$. Using the previous cases we can now proceed by induction as follows: First find a homotopy $F^{0}:[0,1] \times X \rightarrow \Psi(U)$ which is constant outside $W^{0} \subseteq X \times U$ and smooth on $(0,1] \times V^{0}$. By construction, $[0,1] \times W^{1}$ is again a disjoint union of small sets, so we may find a homotopy $F^{1}:[0,1]^{2} \times X \rightarrow \Psi(U)$ starting at $F^{0}$ which is constant outside $[0,1] \times W^{1}$ and smooth on $(0,1] \times[0,1] \times V^{1}$, and so on. In the end we get a map $[0,1]^{k} \times X \rightarrow \Psi(U)$, and we can let $F:[0,1] \times X \rightarrow \Psi(U)$ be the restriction to the diagonal.

\section{The homotopy type of spaces of manifolds}

This section is a self-contained proof of the main theorem of [6].

\subsection{Constructions with tangential structures}

We describe certain constructions that can be made relating $\theta$-manifolds and their submanifolds, using the tangential structure $\theta_{d-1}$ on $(d-1)$-manifolds.

Definition 3.1 If $M \in \Psi_{\theta_{d-1}}\left(\mathbb{R}^{n-1}\right)$, let $\mathbb{R} \times M \subseteq \Psi\left(\mathbb{R}^{n}\right)$ have the $\theta$-structure induced by composing the obvious bundle map

$$
T\left(\mathbb{R}^{1} \times M\right) \rightarrow \varepsilon^{1} \oplus T M
$$


with the bundle map $\varepsilon^{1} \oplus T M \rightarrow \theta^{*} \gamma$ defining the $\theta_{d-1}$-structure on $M$. This defines a map

$$
\begin{aligned}
\Psi_{\theta_{d-1}}\left(\mathbb{R}^{n-1}\right) & \rightarrow \Psi_{\theta}\left(\mathbb{R}^{n}\right) \\
M & \mapsto \mathbb{R} \times M .
\end{aligned}
$$

The construction in Definition 3.1 can be generalised a bit. Let $f: \mathbb{R} \rightarrow \Psi_{\theta_{d-1}}\left(\mathbb{R}^{n-1}\right)$ be a smooth map, having graph $\Gamma(f) \subseteq \mathbb{R} \times \mathbb{R}^{n-1}$. Differentiating the first coordinate $x_{1}: \Gamma(f) \rightarrow \mathbb{R}$ gives a short exact sequence of vector bundles

$$
T^{v}(\Gamma(f)) \rightarrow T(\Gamma(f)) \rightarrow \varepsilon^{1}
$$

and the standard inner product on $\mathbb{R}^{n}$ gives an inner product on $T(\Gamma(f))$, and so a canonical splitting

$$
\varepsilon^{1} \oplus T^{v}(\Gamma(f)) \stackrel{\cong}{\rightarrow} T(\Gamma(f)) .
$$

The $\theta_{d-1}$-structure on each fibre of $\Gamma(f)$ is described by a vector bundle map

$$
\varepsilon^{1} \oplus T^{v}(\Gamma(f)) \rightarrow \theta^{*} \gamma .
$$

Definition 3.2 Let

$$
\begin{aligned}
C^{\infty}\left(\mathbb{R}, \Psi_{\theta_{d-1}}\left(\mathbb{R}^{n-1}\right)\right) & \rightarrow \Psi_{\theta}\left(\mathbb{R}^{n}\right) \\
f & \mapsto \Gamma(f)
\end{aligned}
$$

be the map described above, where $\Gamma(f)$ is given the $\theta$ structure obtained by composing (3-3) with the inverse of (3-2).

The process in Definition 3.1 is the special case of Definition 3.2 where the path $f$ is constant. There is a partially defined reverse process which decreases dimensions of manifolds by one. Let $M \in \Psi_{\theta}\left(\mathbb{R}^{n}\right)$, and again let $x_{1}: M \rightarrow \mathbb{R}$ be the projection to the first coordinate in $\mathbb{R}^{n}$. If $a \in \mathbb{R}$ is a regular value of $x_{1}$, then $M_{a}=M \cap x_{1}^{-1}(a)$ is a smooth manifold and we have a short exact sequence

$$
\left.T M_{a} \rightarrow T M\right|_{M_{a}} \rightarrow \varepsilon^{1} .
$$

Using the inner product on $T M$ induced by $M \subseteq \mathbb{R}^{n}$ we get a splitting

$$
\left.\varepsilon^{1} \oplus T M_{a} \stackrel{\cong}{\rightarrow} T M\right|_{M_{a}} \text {. }
$$

Definition 3.3 Let

$$
\begin{aligned}
& \Psi_{\theta}\left(\mathbb{R}^{n}\right)-\rightarrow \Psi_{\theta_{d-1}}\left(\mathbb{R}^{n-1}\right) \\
& M \mapsto M_{a}=M \cap x_{1}^{-1}(a)
\end{aligned}
$$

be the partially defined map which gives $M_{a}$ the $\theta$-structure induced by composing (3-4) with the bundle map $T M \rightarrow \theta^{*} \gamma$ defining the $\theta$-structure on $M$. 
Finally we discuss an extended functoriality of $\Psi_{\theta}$. Let $M \in \Psi_{\theta}\left(\mathbb{R}^{n}\right)$ and let $F: \mathbb{R}^{n} \rightarrow$ $\mathbb{R}^{n}$ be a map which is transverse to $M$. Then $W=F^{-1} M$ is again a $d$-manifold, but in general has no induced $\theta$-structure. For example if $F: \mathbb{R}^{3} \rightarrow \mathbb{R}^{3}$ given by $F(x)=\left(0,0,|x|^{2}\right)$ then $F^{-1}\left(\mathbb{R}^{2} \times\{1\}\right)=S^{2}$, but the canonical framing of $\mathbb{R}^{2} \times\{1\}$ cannot induce a framing of $S^{2}$.

It is, however, possible to define such an induced structure in special cases. The following will be needed below. Let $\varphi: \mathbb{R} \rightarrow \mathbb{R}$ be a smooth map with $\varphi^{\prime} \geq 0$. Let $F=\varphi \times \mathrm{Id}: \mathbb{R}^{n} \rightarrow \mathbb{R}^{n}$. If $M \in \Psi\left(\mathbb{R}^{n}\right)$ and $F$ is transverse to $M$, then $W=F^{-1}(M)$ is again a $d$-manifold. Both bundles $T W$ and $F^{*}(T M)$ are subbundles of $\varepsilon^{n}$, and they are related via the endomorphism $D F: \varepsilon^{n} \rightarrow \varepsilon^{n}$ by the equation

$$
T W=D F^{-1}\left(F^{*} T M\right) .
$$

An exercise in linear algebra shows that the composition

$$
T W \hookrightarrow \varepsilon^{n} \rightarrow F^{*} T M,
$$

the inclusion followed by orthogonal projection, defines an isomorphism $T W \rightarrow$ $F^{*} T M$. Using this isomorphism to give $W$ an induced $\theta$-structure, we get a partially defined map

$$
\begin{aligned}
\Psi_{\theta}\left(\mathbb{R}^{n}\right) & -\rightarrow \Psi_{\theta}\left(\mathbb{R}^{n}\right) \\
M & \mapsto F^{-1} M=(\varphi \times \mathrm{Id})^{-1}(M) .
\end{aligned}
$$

A typical application of this construction is given by the following lemma. We say that an $M \in \Psi_{\theta}\left(\mathbb{R}^{n}\right)$ is cylindrical in $x_{1}^{-1}(a, b)$ if there is an $N \in \Psi_{\theta_{d-1}}\left(\mathbb{R}^{n-1}\right)$ such that

$$
\left.(\mathbb{R} \times N)\right|_{x_{1}^{-1}(a, b)}=\left.M\right|_{x_{1}^{-1}(a, b)} \in \Psi_{\theta}\left(x_{1}^{-1}(a, b)\right) .
$$

Lemma 3.4 Let $f: X \rightarrow \Psi_{\theta}\left(\mathbb{R}^{n}\right)$ be continuous, and let $U, V \subseteq X$ be open, with $\bar{U} \subseteq V$. Let $a \in \mathbb{R}$ be a regular value for $x_{1}: f(x) \rightarrow \mathbb{R}$ for all $x \in V$. Let $\varepsilon>0$. Then there is a homotopy

$$
f_{t}: X \rightarrow \Psi_{\theta}\left(\mathbb{R}^{n}\right), \quad t \in[0,1]
$$

with $f_{0}=f$, and:

(i) $f_{1}(x)$ cylindrical in $x_{1}^{-1}([a-\varepsilon, a+\varepsilon])$ for $x \in U$.

(ii) The restriction to $[0,1] \times(X-V) \rightarrow \Psi_{\theta}\left(\mathbb{R}^{n}\right)$ is a constant homotopy.

(iii) The composition $[0,1] \times X \rightarrow \Psi_{\theta}\left(\mathbb{R}^{n}\right) \rightarrow \Psi_{\theta}\left(\mathbb{R}^{n}-x_{1}^{-1}([a-2 \varepsilon, a+2 \varepsilon])\right)$ is a constant homotopy. 
Proof Choose once and for all a smooth function $\lambda: \mathbb{R} \rightarrow \mathbb{R}$ with $\lambda(s)=0$ for $|s| \leq 1, \lambda(s)=s$ for $|s| \geq 2$, and $\lambda^{\prime}(s)>0$ for $|s|>1$. For $\tau \in[0,1]$, let $\varphi_{\tau}(s)=$ $(1-\tau) s+\tau \varepsilon \lambda((s-a) / \varepsilon)$. Then pick a function $\rho: X \rightarrow[0,1]$ with $\operatorname{supp}(\rho) \subseteq V$ and $U \subseteq \rho^{-1}(1)$ and define the homotopy by

$$
\begin{aligned}
{[0,1] \times X } & \rightarrow \Psi_{\theta}\left(\mathbb{R}^{n}\right) \\
(t, x) & \mapsto\left(\varphi_{t \rho(x)} \times \mathrm{Id}\right)^{-1}(f(x)) .
\end{aligned}
$$

Finally, let us introduce some subspaces of $\Psi_{\theta}\left(\mathbb{R}^{n}\right)$ which are very important in the sequel.

Definition 3.5 Let $\psi_{\theta}(n, k) \subseteq \Psi_{\theta}\left(\mathbb{R}^{n}\right)$ be the subspace defined by the condition $M \subseteq \mathbb{R}^{k} \times(-1,1)^{n-k}$.

Now $\psi_{\theta_{d-1}}(n-1, k-1) \subseteq \Psi_{\theta_{d-1}}\left(\mathbb{R}^{n-1}\right)$ is the subspace defined by the condition $M \subseteq \mathbb{R}^{k-1} \times(-1,1)^{n-k}$ and we see that the map (3-1) and the partially defined map (3-5) restrict to maps

$$
\begin{aligned}
& \psi_{\theta_{d-1}}(n-1, k-1) \rightarrow \psi_{\theta}(n, k) \\
& M \mapsto \mathbb{R} \times M, \\
& \psi_{\theta}(n, k)-\rightarrow \psi_{\theta_{d-1}}(n-1, k-1) \\
& M \mapsto M_{a}=M \cap x_{k}^{-1}(a) .
\end{aligned}
$$

Proposition 3.6 For $k>1$, the map (3-7) induces an isomorphism on $\pi_{0}$, with inverse induced by (3-8). Consequently $\pi_{0}\left(\psi_{\theta}(n, k)\right) \cong \pi_{0}\left(\psi_{\theta_{d-k+1}}(n-k+1,1)\right)$.

Proof By Sard's theorem, every element $M \in \psi_{\theta}(n, k)$ is in the domain of the map (3-8) for some $a$. We will show that the element $\left[M_{a}\right] \in \pi_{0}\left(\psi_{\theta_{d-1}}(n-1, k-1)\right)$ is independent of $a$.

Let $M \in \psi_{\theta}(n, k)$ and $a<b$ be two regular values of $x_{1}: M \rightarrow \mathbb{R}$. Near $x_{1}^{-1}(a)$ and $x_{1}^{-1}(b)$, the function $x_{1}$ has no critical points. We can perturb it, relative to these subsets, to be a Morse function (as they are dense in the space of smooth functions) and still give an embedding (as the space of embeddings is open in the space of all smooth maps). We then obtain a manifold $M^{\prime}$, such that $x_{1}$ has isolated critical points. We may then perturb $M^{\prime}$ slightly so that there are no critical points in $[a, b] \times\{0\}^{k-1} \times[-1,1]^{n-k}$. Let us still call this $M^{\prime}$. As critical points are isolated, there is an $\varepsilon>0$ such that there are no critical points in $[a, b] \times(-\varepsilon, \varepsilon)^{k-1} \times[-1,1]^{n-k}$. Choosing an isotopy of embeddings $e_{t}: \mathbb{R} \rightarrow \mathbb{R}$ from the identity to a diffeomorphism onto $(-\varepsilon, \varepsilon)$, we can form $h_{t}=\mathbb{R} \times e_{t}^{k-1} \times[-1,1]^{n-k}$ and let $M^{\prime}(t)=h_{t}^{-1}\left(W^{\prime}\right)$. 
This gives a path from $M^{\prime}=M^{\prime}(0)$ to a manifold $M^{\prime}(1)$ such that $x_{1}$ has no critical values in $[a, b]$. Thus there are paths

$$
M_{a}=M_{a}^{\prime} \stackrel{M_{a}^{\prime}(t)}{\rightsquigarrow} M_{a}^{\prime}(1) \stackrel{M_{t}^{\prime}(1)}{\rightsquigarrow} M_{b}^{\prime}(1) \stackrel{M_{b}^{\prime}(t)}{\rightsquigarrow} M_{b}^{\prime}=M_{b} .
$$

We have shown that (3-8) gives a well defined map $\psi_{\theta}(n, k) \rightarrow \pi_{0}\left(\psi_{\theta_{d-1}}(n-1, k-1)\right)$. This map is locally constant so it factors through $\pi_{0}\left(\psi_{\theta}(n, k)\right)$. The composition

$$
\pi_{0}\left(\psi_{\theta_{d-1}}(n-1, k-1)\right) \rightarrow \pi_{0}\left(\psi_{\theta}(n, k)\right) \rightarrow \pi_{0}\left(\psi_{\theta_{d-1}}(n-1, k-1)\right)
$$

is the identity map, so the first map is injective. To see surjectivity let $M \in \psi_{\theta}(n, k)$ and $a$ be a regular value of $x_{1}: M \rightarrow \mathbb{R}$. Similarly to the proof of Lemma 3.4, let $\varphi_{t}: \mathbb{R} \rightarrow \mathbb{R}, t \in[0,1]$ be given by $\varphi_{t}(s)=(1-t) \cdot(s-a)+a$. Then $M(t)=$ $\left(\varphi_{t} \times \mathrm{Id}\right)^{-1}(W)$ gives a path from $M$ to the cylindrical manifold $\mathbb{R} \times M_{a}$, where tangential structures are handled using the extended functoriality of (3-6). Thus the first map is also surjective, and so a bijection.

\subsection{The cobordism category}

Let us give a definition of the embedded cobordism category $C_{\theta}\left(\mathbb{R}^{n}\right)$ from [6], using the topological sheaf $\Psi_{\theta}$ from the previous section. There are several versions of the definition, but they all give level-wise homotopy equivalent categories, where we say a functor $F: \mathcal{C} \rightarrow \mathcal{D}$ is a level-wise homotopy equivalence of categories if $N_{k} F: N_{k} \mathcal{C} \rightarrow N_{k} \mathcal{D}$ is a homotopy equivalence for all $k$.

Definition 3.7 Let $\mathcal{C}_{\theta}\left(\mathbb{R}^{n}\right)$ have object space $\psi_{\theta_{d-1}}(n-1,0)$. The set of nonidentity morphisms from $M_{0}$ to $M_{1}$ is the set of $(t, W) \in \mathbb{R} \times \psi_{\theta}(n, 1)$ such that $t>0$ and such that there exists an $\varepsilon>0$ such that

$$
\begin{aligned}
\left.W\right|_{(-\infty, \varepsilon) \times \mathbb{R}^{n-1}} & =\left.\left(\mathbb{R} \times M_{0}\right)\right|_{(-\infty, \varepsilon) \times \mathbb{R}^{n-1}} \in \Psi_{\theta}\left((-\infty, \varepsilon) \times \mathbb{R}^{n-1}\right) \\
\left.W\right|_{(t-\varepsilon, \infty) \times \mathbb{R}^{n-1}} & =\left.\left(\mathbb{R} \times M_{1}\right)\right|_{(t-\varepsilon, \infty) \times \mathbb{R}^{n-1}} \in \Psi_{\theta}\left((t-\varepsilon, \infty) \times \mathbb{R}^{n-1}\right),
\end{aligned}
$$

where $\mathbb{R} \times M_{v} \in \Psi_{\theta}\left(\mathbb{R}^{n}\right)$ is as explained in (3-1). Composition in the category is defined by

$$
(t, W) \circ\left(t^{\prime}, W^{\prime}\right)=\left(t+t^{\prime}, W^{\prime \prime}\right),
$$

where $W^{\prime \prime}$ agrees with $W$ near $(-\infty, t] \times \mathbb{R}^{n-1}$ and with $t \cdot e_{1}+W^{\prime}$ near $[t, \infty) \times \mathbb{R}^{n-1}$. The total space of morphisms is topologised as a subspace of $(\{0\} \amalg(0, \infty)) \times \psi_{\theta}(n, 1)$, where $(0, \infty)$ is given the usual topology.

Of course, the important part of a morphism $\left(a_{0}<a_{1}, M\right)$ is the part of $M$ that lies in $\left[a_{0}, a_{1}\right] \times \mathbb{R}^{n-1}$, since it uniquely determines the rest of $M$.

The second version of the definition of the cobordism category is a topological poset. 


\section{Definition 3.8 Let}

$$
D_{\theta}\left(\mathbb{R}^{n}\right) \subseteq \mathbb{R} \times \psi_{\theta}(n, 1)
$$

denote the space of pairs $(t, M)$ such that $t$ is a regular value of $x_{1}: M \rightarrow \mathbb{R}$. Topologise $D_{\theta}\left(\mathbb{R}^{n}\right)$ as a subspace and order it by declaring $(t, M) \leq\left(t^{\prime}, M^{\prime}\right)$ if and only if $M=M^{\prime}$ and $t \leq t^{\prime}$.

Theorem 3.9 There is a zig-zag

$$
\mathcal{C}_{\theta}\left(\mathbb{R}^{n}\right) \stackrel{c}{\longleftarrow} D_{\theta}^{\perp}\left(\mathbb{R}^{n}\right) \stackrel{i}{\rightarrow} D_{\theta}\left(\mathbb{R}^{n}\right)
$$

of level-wise homotopy equivalences of categories. In particular, the classifying spaces $B \mathcal{C}_{\theta}\left(\mathbb{R}^{n}\right)$ and $B D_{\theta}$ are homotopy equivalent.

Proof We must first describe $D_{\theta}^{\perp}\left(\mathbb{R}^{n}\right)$. It is defined as $D_{\theta}\left(\mathbb{R}^{n}\right)$, except that we only allow pairs $(t, M)$ such that $M$ is cylindrical in $x_{1}^{-1}(t-\varepsilon, t+\varepsilon)$ for some $\varepsilon>0$. The functor $D_{\theta}^{\perp}\left(\mathbb{R}^{n}\right) \rightarrow D_{\theta}\left(\mathbb{R}^{n}\right)$ is by inclusion. It is a levelwise homotopy equivalence on simplicial nerves by Lemma 3.4.

Let us digress for a moment to a construction similar to (3-6). Let $\varphi_{s}(a, b)$ be the function as in Figure 1, where we allow $s=\infty$ to be the obvious limit. Note that it is smooth away from the set of points $\{a-s, a, b, b+s\}$, and a diffeomorphism on $(-\infty, a-s] \cup[a, b] \cup[b+s, \infty)$.

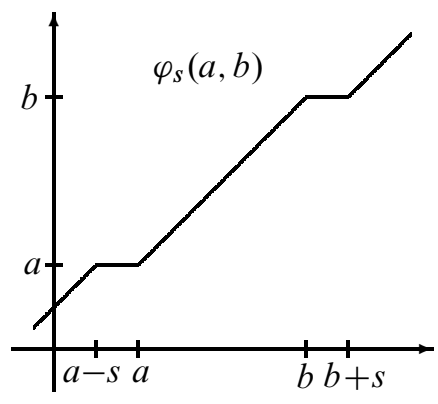

Figure 1

Suppose $M \in \psi_{\theta}(n, 1)$ is cylindrical near $a$ and $b$, then $\left(\varphi_{s}(a, b) \times \operatorname{Id}\right)^{-1}(M)$ defines an element of $\psi_{\theta}(n, 1)$ as follows. It certainly defines a smooth submanifold of $\mathbb{R} \times(-1,1)^{n-1}$ that agrees with the underlying manifold of $M$ inside $x_{1}^{-1}(a, b)$, is cylindrical in $x_{1}^{-1}(a-s, a)$ and $x_{1}^{-1}(b, b+s)$, and agrees with translated copies of the underlying manifold $M$ on $x_{1}^{-1}(-\infty, a-s]$ and $x_{1}^{-1}[b+s, \infty)$. The $\theta$-structure is defined to be that of $M$ on $x_{1}^{-1}((-\infty, a-s) \cup(a, b) \cup(b+s, \infty))$ where there are 
diffeomorphisms to $M$. On $x_{1}^{-1}[a-s, a]$ the underlying manifold is cylindrical and so agrees with $\mathbb{R} \times x_{1}^{-1}\{a\}$. The $\theta$-structure on $x_{1}^{-1}(\{a-s, a\})$ also agrees, so we can use this identification to give a $\theta$-structure on $x_{1}^{-1}[a-s, a]$. Similarly $x_{1}^{-1}[b, b+s]$. The functor $D_{\theta}^{\perp}\left(\mathbb{R}^{n}\right) \rightarrow \mathcal{C}_{\theta}\left(\mathbb{R}^{n}\right)$ sends an object $(t, M)$ to $x_{1}^{-1}(t)$ as in (3-5). On nonidentity morphisms it is

$$
\left(t_{0}<t_{1}, M\right) \mapsto\left(t_{1}-t_{0},\left(\varphi_{\infty}\left(t_{0}, t_{1}\right) \times \mathrm{Id}\right)^{-1}(M)-t_{0} \cdot e_{1}\right),
$$

where $e_{1} \in \mathbb{R}^{n}$ is the first basis vector and $-t_{0} \cdot e_{1}$ denotes the parallel translation.

On simplicial nerves there is a map in the reverse direction, $N_{l} \mathcal{C}_{\theta}\left(\mathbb{R}^{n}\right) \rightarrow N_{l} D_{\theta}^{\perp}\left(\mathbb{R}^{n}\right)$, that is the inclusion of those $\left(0=t_{0}<t_{1}<\cdots<t_{l}, M\right)$ such that $M$ is cylindrical on $x_{1}^{-1}(-\infty, 0)$ and on $x_{1}^{-1}\left(t_{l}, \infty\right)$. The functor can then be viewed as the self map $h_{1}$ of $N_{l} D_{\theta}^{\perp}\left(\mathbb{R}^{n}\right)$ that sends $\left(t_{0}<t_{1}<\cdots<t_{l}, M\right)$ to

$$
\left(0<t_{1}-t_{0}<\cdots<t_{l}-t_{0},\left(\varphi_{\infty}\left(t_{0}, t_{l}\right) \times \mathrm{Id}\right)^{-1}(M)-t_{0} \cdot e_{1}\right) .
$$

This is isotopic to the identity via the homotopy $h_{s}$ that sends $\left(t_{0}<t_{1}<\cdots<t_{l}, M\right)$ to

$$
\left(t_{0}-s \cdot t_{0}<t_{1}-s \cdot t_{0}<\cdots<t_{l}-s \cdot t_{0},\left(\varphi_{s /(1-s)}\left(t_{0}, t_{l}\right) \times \mathrm{Id}\right)^{-1}(M)-s \cdot t_{0} \cdot e_{1}\right) .
$$

Finally we determine the homotopy type of $B \mathcal{C}_{\theta}\left(\mathbb{R}^{n}\right) \simeq B D_{\theta}\left(\mathbb{R}^{n}\right)$.

Theorem 3.10 The forgetful map induces a weak equivalence

$$
B D_{\theta}\left(\mathbb{R}^{n}\right) \stackrel{u}{\rightarrow} \psi_{\theta}(n, 1) .
$$

Proof The inverse image of a point $M \in \psi_{\theta}(n, 1)$ is a subspace of the infinite simplex $B(\mathbb{R}, \leq)$. It is the simplex whose vertices is the space of regular values of $x_{1}: M \rightarrow \mathbb{R}$. Points in the inverse image of $M$ can be represented as formal affine combinations (ie formal linear combinations where the coefficients are nonnegative and sum to 1) of regular values of $x_{1}$. This inverse image is contractible (the space of vertices is nonempty by Sard's theorem) which suggests the map might be a weak equivalence. To give a rigorous proof we calculate the relative homotopy groups.

Let

$$
\begin{gathered}
f: D^{m} \rightarrow \psi_{\theta}(n, 1) \\
\widehat{f}: \partial D^{m} \rightarrow B D_{\theta}\left(\mathbb{R}^{n}\right)
\end{gathered}
$$

be continuous maps with $u \circ \widehat{f}=\left.f\right|_{\partial D^{m}}$.

For $a \in \mathbb{R}$, let $U_{a} \subseteq D^{m}$ be the set of points $x$ such that $a$ is a regular value of $x_{1}: f(x) \rightarrow \mathbb{R}$. This is an open subset of $D^{m}$, so by compactness we can pick 
finitely many $a_{1}, \ldots, a_{k} \in \mathbb{R}$ such that the $U_{a_{i}}$ cover $D^{m}$. Pick a partition of unity $\lambda_{1}, \ldots, \lambda_{k}: D^{m} \rightarrow[0,1]$ subordinate to the cover. Using $\lambda_{i}$ as a formal coefficient of $a_{i}$ gives a map

$$
g: D^{m} \rightarrow B D_{\theta}\left(\mathbb{R}^{n}\right)
$$

which lifts $f$, ie $u \circ g=f$. Finally we produce a homotopy between the two maps $\left.g\right|_{\partial D^{m}}$ and $\hat{f}$. Since they are both lifts of $\left.f\right|_{\partial D^{m}}$, we can just use the affine structure on the fibres of $u$ to give the straight-line homotopy.

This proves that the relative homotopy groups (of $B D_{\theta}\left(\mathbb{R}^{n}\right.$ ) as a subspace of the mapping cylinder of $u$ ) vanish and hence the map $u$ is a weak equivalence.

We can now calculate the set of path components of $\psi_{\theta}(n, 1)$. We can define a product of two elements $W_{1}$ and $W_{2}$ of the space $\psi_{\theta}(n, 1)$ in the following way. Take the union of the disjoint manifolds $W_{1}$ and $W_{2}+e_{2}$ and scale the second coordinate by $1 / 2$. This product

$$
\psi_{\theta}(n, 1) \times \psi_{\theta}(n, 1) \rightarrow \psi_{\theta}(n, 1)
$$

makes $\psi_{\theta}(n, 1)$ into an $H$-space (in fact it is an $E_{n-1}$ space) and hence $\pi_{0}\left(\psi_{\theta}(n, 1)\right)$ is a monoid. We have the following corollary.

Corollary 3.11 The monoid $\pi_{0} \psi_{\theta}(n, 1)$ is isomorphic to the monoid of cobordism classes of $\theta_{d-1}$ manifolds in $\mathbb{R}^{n-1}$. In particular the monoid is a group.

Proof By Theorem 3.10 and Proposition 3.6 we have

$$
\pi_{0}\left(\psi_{\theta}(n, 1)\right)=\pi_{0}\left(B D_{\theta}\left(\mathbb{R}^{n}\right)\right)=\pi_{0}\left(B \mathcal{C}_{\theta}\left(\mathbb{R}^{n}\right)\right),
$$

which can be identified with the set objects of $\mathcal{C}_{\theta}$, modulo the equivalence relation generated by the morphisms. This proves the first claim.

The monoid structure on $\pi_{0}\left(B \mathcal{C}_{\theta}\left(\mathbb{R}^{n}\right)\right)$ comes from an $H$-space structure on the objects and morphisms of $\mathcal{C}_{\theta}$, defined by disjoint union in the second coordinate direction of $\mathbb{R}^{n}$, as for $\psi_{\theta}(n, 1)$. To see that it is a group, let $M \in \psi_{\theta_{d-1}}(n-1,0)$ be an object, and let $\mathbb{R} \times M \in \psi_{\theta}(n, 1)$ be the corresponding cylindrical element. There is another object " $-M$ ", such that $\mathbb{R} \times(-M)$ is obtained from $\mathbb{R} \times M$ by changing signs of the first two coordinates in $\mathbb{R}^{n}$.

There is an embedding $e$ of $\mathbb{R} \times I$ into itself given by Figure 2 . Then $\left(e \times I^{n-2}\right)(\mathbb{R} \times M)$ gives a morphism from $M \sqcup-M$ to the empty manifold, showing that $[M]$ and $[-M]$ are inverse points in the monoid structure on $\pi_{0}\left(B \mathcal{C}_{\theta}\left(\mathbb{R}^{n}\right)\right)$. 


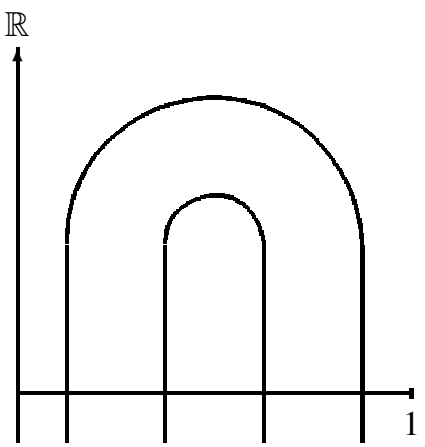

Figure 2

\subsection{The homotopy type of the space of all $\theta$-manifolds}

In this section we finish our proof of the main theorem of [6]. There is a space $\mathbf{X}\left(\mathbb{R}^{n}\right)$ defined by the fibre product

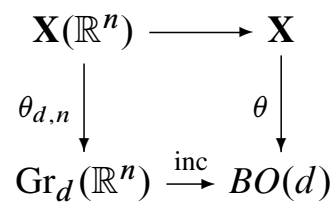

where we consider $B O(d)$ as the infinite Grassmannian $\mathrm{Gr}_{d}\left(\mathbb{R}^{\infty}\right)$ and the inclusion map as that induced by $\mathbb{R}^{n} \subseteq \mathbb{R}^{\infty}$. Thus $\mathbf{X}\left(\mathbb{R}^{n}\right)$ is simply that part of $\mathbf{X}$ which lies over those $d$-planes which are contained in $\mathbb{R}^{n} \subseteq \mathbb{R}^{\infty}$.

Theorem 3.12 There is a weak homotopy equivalence

$$
B \mathcal{C}_{\theta}\left(\mathbb{R}^{n}\right) \simeq \psi_{\theta}(n, 1) \stackrel{\simeq}{\rightarrow} \Omega^{n-1} \operatorname{Th}\left(\theta_{d, n}^{*}\left(\gamma_{d, n}^{\perp}\right) \rightarrow \mathbf{X}\left(\mathbb{R}^{n}\right)\right) .
$$

Letting $n$ go to infinity, we get the main theorem of [6],

$$
B \mathcal{C}_{\theta} \simeq \psi_{\theta}(\infty, 1) \stackrel{\simeq}{\rightarrow} \Omega^{\infty-1} \mathbf{M T} \theta .
$$

Theorem 3.12 will be proved in Theorems 3.13 and 3.22 below. First we construct the relevant maps. Consider the map

$$
\begin{aligned}
\mathbb{R} \times \psi_{\theta}(n, k-1) & \rightarrow \psi_{\theta}(n, k) \\
(t, M) & \mapsto M-t \cdot e_{k},
\end{aligned}
$$

where $e_{k} \in \mathbb{R}^{n}$ is the $k$-th standard basis vector and $M-t \cdot e_{k}$ denotes the inverse image of $M$ under the diffeomorphism $x \mapsto x+t \cdot e_{k}$. This is a continuous map of spaces as it is induced by the action of the subgroup $\mathbb{R} \subseteq \operatorname{Diff}\left(\mathbb{R}^{n}\right)$. 
If we let $\varnothing \in \psi_{\theta}(n, k)$ be the basepoint, then (3-9) extends uniquely to a continuous map $S^{1} \wedge \psi_{\theta}(n, k-1) \rightarrow \psi_{\theta}(n, k)$ with adjoint map

$$
\psi_{\theta}(n, k-1) \rightarrow \Omega \psi_{\theta}(n, k) .
$$

Theorem 3.13 The map (3-10) is a homotopy equivalence for $k \geq 2$. Consequently there is a homotopy equivalence

$$
\psi_{\theta}(n, 1) \stackrel{\simeq}{\longrightarrow} \Omega^{n-1} \psi_{\theta}(n, n) .
$$

The homotopy equivalence (3-10) will be proved in three steps, Propositions 3.19, 3.20 and 3.21. Let $k \geq 2$ be fixed. The idea of the proof is to instead prove a homotopy equivalence $B \psi_{\theta}(n, k-1) \simeq \psi_{\theta}(n, k)$ for a suitable "monoid structure" on $\psi_{\theta}(n, k-1)$. In fact it is convenient to work with something which is not quite a monoid structure, but which contains the same homotopical information. We recall the following well known lemma.

Lemma 3.14 Let $X$ • be a simplicial space such that the face maps induce a homotopy equivalence $X_{k} \simeq X_{1} \times \cdots \times X_{1}$. (When $k=0$ this means that $X_{0}$ is contractible). Then the natural map

$$
X_{1} \rightarrow \Omega\left\|X_{\bullet}\right\|
$$

is a homotopy equivalence if and only if $X_{\bullet}$ is group-like ie $\pi_{0} X_{1}$ is a group with respect to the product induced by $d_{1}: X_{2} \rightarrow X_{1}$.

Proof Here $\Omega\left\|X_{\bullet}\right\|$ should be taken to mean the space of paths in the fat realisation $\left\|X_{\bullet}\right\|$ which start and end somewhere in $X_{0} \subseteq\left\|X_{\bullet}\right\|$. This space receives a natural map from $X_{1}$. The lemma is a well known variant of the fact that $M \simeq \Omega B M$ if $M$ is a group-like topological monoid. See Segal [13, Proposition 1.5].

Given any pair $a, b \in X_{0}$, the space $\Omega_{a, b}\left\|X_{\bullet}\right\|$ of paths starting at $a$ and ending at $b$ is homotopy equivalent to $\Omega\left\|X_{\bullet}\right\|$ defined above.

Instead of defining an actual monoid structure on $\psi_{\theta}(n, k-1)$ we can define a simplicial space satisfying the lemma. By abuse of notation we will call it $N_{\bullet} \psi_{\theta}(n, k-1)$ since it plays the role of the nerve of a monoid. First we have a preliminary definition.

Definition 3.15 Let $A \subseteq \mathbb{R}$ be an open subset, and $x_{k}: \mathbb{R}^{n} \rightarrow \mathbb{R}$ denote the projection on the $k$-th coordinate. Let $\psi_{\theta}^{A}(n, k) \subseteq \psi_{\theta}(n, k)$ denote the subset defined by requiring $M \cap x_{k}^{-1}(\mathbb{R}-A)=\varnothing$. In particular $\psi_{\theta}(n, k-1)=\psi_{\theta}^{(-1,1)}(n, k)$.

Let $\psi_{\theta}^{A}(n, k)^{\prime} \subseteq \psi_{\theta}(n, k)$ be the subset defined by the condition $M \cap[-1,1]^{n} \cap$ $x_{k}^{-1}(\mathbb{R}-A)=\varnothing$. 


\section{Lemma 3.16}

(i) The inclusion $\psi_{\theta}^{A}(n, k) \rightarrow \psi_{\theta}^{A}(n, k)^{\prime}$ is a homotopy equivalence.

(ii) If $A_{1}, \ldots, A_{l} \subseteq \mathbb{R}$ are disjoint open sets and $A=\cup A_{i}$ then union of subsets defines a homeomorphism

$$
\prod \psi_{\theta}^{A_{i}}(n, k) \stackrel{\cong}{\rightarrow} \psi_{\theta}^{A}(n, k) .
$$

(iii) If $A=\left(a_{0}, a_{1}\right)$ is an open interval then $\psi_{\theta}^{A}(n, k) \cong \psi_{\theta}(n, k-1)$.

(iv) If $A=(-\infty, a)$ or $A=(a, \infty)$ for some $a \in \mathbb{R}$, then $\psi_{\theta}^{A}(n, k)$ and $\psi_{\theta}^{A}(n, k)^{\prime}$ are contractible.

Proof For part (i), we prove that the inclusion is a deformation retract. Pick an isotopy $e_{t}: \mathbb{R} \rightarrow \mathbb{R}, t \in[0,1]$ of embeddings, with $e_{0}$ the identity and $e_{1}(\mathbb{R})=(-1,1)$. Let

$$
j_{t}=\left(e_{t}\right)^{k-1} \times(\mathrm{Id})^{n-k+1}: \mathbb{R}^{n} \rightarrow \mathbb{R}^{n} .
$$

This gives a path $[0,1] \rightarrow \operatorname{Emb}\left(\mathbb{R}^{n}, \mathbb{R}^{n}\right)$, and we define a homotopy

$$
\psi_{\theta}(n, k) \stackrel{h_{t}}{\longrightarrow} \psi_{\theta}(n, k), \quad t \in[0,1]
$$

by $h_{t}(M)=e_{t}^{-1}(M)$. This restricts to a deformation retraction because the homotopy preserves $\psi_{\theta}^{A}(n, k)$ and $\psi_{\theta}^{A}(n, k)^{\prime}$, starts at the identity, and $h_{1}$ maps $\psi_{\theta}^{A}(n, k)^{\prime}$ into $\psi_{\theta}^{A}(n, k)$.

Part (ii) is obvious and for (iii), a homeomorphism is obtained by stretching the $k$-th coordinate using the affine map $f: \mathbb{R} \rightarrow \mathbb{R}$ with $f\left(a_{0}\right)=-1$ and $f\left(a_{1}\right)=1$. For (iv) it suffices to consider $\psi_{\theta}^{A}(n, k)$ with $A=(-\infty, a)$. We define a homotopy by

$$
\begin{aligned}
{[0,1] \times \psi_{\theta}^{A}(n, k) } & \rightarrow \psi_{\theta}^{A}(n, k) \\
(t, M) & \mapsto M-\frac{t}{1-t} \cdot e_{k} \\
(1, M) & \mapsto \varnothing .
\end{aligned}
$$

This contracts $\psi_{\theta}^{A}(n, k)$ to the point $\varnothing$.

Definition 3.17 Denote by $N_{\bullet} \psi_{\theta}(n, k-1)$ the simplicial space defined by letting $N_{l} \psi_{\theta}(n, k-1) \subseteq \mathbb{R}^{l+1} \times \psi_{\theta}(n, k)$ be the set of pairs $(t, M)$ such that $0<t_{0} \leq \cdots \leq$ $t_{l}<1$ and such that $M \in \psi_{\theta}^{A}(n, k)$ with $A=\mathbb{R}-\left\{t_{0}, \ldots t_{l}\right\}$.

Lemma 3.18 $N_{\bullet} \psi_{\theta}(n, k-1)$ satisfies the assumptions of Lemma 3.14.

Proof This follows immediately from Lemma 3.16. 
It remains to see that $\pi_{0} N_{1} \psi_{\theta}(n, k-1)$ is a group, but this follows from Corollary 3.11. We have proved:

Proposition 3.19 The natural map

$$
\psi_{\theta}(n, k-1) \rightarrow \Omega\left\|N_{\bullet} \psi_{\theta}(n, k-1)\right\|
$$

is a homotopy equivalence.

Proposition 3.20 The forgetful map $(t, M) \mapsto M$ defines a homotopy equivalence

$$
\left\|N \bullet \psi_{\theta}(n, k-1)\right\| \rightarrow \psi_{\theta}^{0}(n, k),
$$

where $\psi_{\theta}^{0}(n, k) \subseteq \psi_{\theta}(n, k)$ is the subset where $M$ satisfies $M \cap[-1,1]^{n} \cap x_{k}^{-1}(t)=\varnothing$ for some $t \in(0,1)$.

Proof There is a simplicial space $N_{\bullet} \psi_{\theta}(n, k-1)^{\prime}$ defined as in Definition 3.17, but with $\psi_{\theta}^{A}(n, k-1)^{\prime}$ instead of $\psi_{\theta}^{A}(n, k-1)$. By Lemma 3.16(i), the inclusion $N_{\bullet} \psi_{\theta}(n, k-1) \rightarrow N_{\bullet} \psi_{\theta}(n, k-1)^{\prime}$ is a levelwise homotopy equivalence, so it suffices to prove that $\left\|N_{\bullet} \psi_{\theta}(n, k-1)^{\prime}\right\| \rightarrow \psi_{\theta}^{0}(n, k)$ is a homotopy equivalence.

The fibre over $M \in \psi_{\theta}^{0}(n, k)$ is the classifying space of the poset of $t \in(0,1)$ such that $M \cap[-1,1]^{n} \cap x_{k}^{-1}(t)=\varnothing$, ordered as usual. This is a totally ordered nonempty set, so the realisation is a simplex. Therefore the map is a weak homotopy equivalence as in the proof of Theorem 3.10.

Proposition 3.21 Let $\psi_{\theta}^{\varnothing}(n, k) \subseteq \psi_{\theta}(n, k)$ denote the path component of the empty set. Then the inclusion

$$
\psi_{\theta}^{0}(n, k) \rightarrow \psi_{\theta}^{\varnothing}(n, k)
$$

is a weak homotopy equivalence.

Proof For ease of notation we will switch the roles of the coordinates $x_{1}$ and $x_{k}$ in this proof. Thus $\psi_{\theta}^{0}(n, k)$ becomes the subspace consisting of manifolds $M$ satisfying $M \cap x_{1}^{-1}(a) \cap[-1,1]^{n}=\varnothing$ for some $a$. We prove that the relative homotopy groups vanish. Let

$$
f:\left(D^{m}, \partial D^{m}\right) \rightarrow\left(\psi_{\theta}^{\varnothing}(n, k), \psi_{\theta}^{0}(n, k)\right)
$$

represent an element of relative $\pi_{m}$. We may assume $f$ is smooth.

For each $a \in \mathbb{R}$, let $U_{a} \subseteq D^{m}$ the set of points $y \in D^{m}$ such that $x_{1}: f(y) \rightarrow \mathbb{R}$ has no critical points in $\{a\} \times I^{k-1} \times \mathbb{R}^{n-k}$. This is an open condition on $f(y)$, so all $U_{a} \subseteq D^{m}$ are open. As in the proof of Lemma 3.16(i), pick an isotopy of embeddings $e_{t}: \mathbb{R} \rightarrow \mathbb{R}, t \in[0,1]$ starting at $e_{0}=\mathrm{Id}$ and ending at a diffeomorphism 
$e_{1}: \mathbb{R} \rightarrow(-1,1)$. Let $h_{t}: \mathbb{R}^{n} \rightarrow \mathbb{R}^{n}$ be given by $h_{t}=\mathrm{Id} \times e_{t}^{k-1} \times \mathrm{Id}$ and define a homotopy $f_{t}: D^{m} \rightarrow \psi_{\theta}^{\varnothing}(n, k), t \in[0,1]$ by

$$
f_{t}(x)=h_{t}^{-1}(f(x)) .
$$

This gives a relative homotopy that starts at $f_{0}=f$, and ends at a map $f_{1}$, where in $f_{1}(x)=h_{1}^{-1}(f(x))$ we have "stretched" the space

$$
\{a\} \times(-1,1)^{k-1} \times \mathbb{R}^{n-k}
$$

to be all of $\{a\} \times \mathbb{R}^{n-1}$. Therefore $x_{1}: f_{1}(x) \rightarrow \mathbb{R}$ now has no critical points in $\{a\} \times \mathbb{R}^{n-1}$ for all $x \in U_{a}$. We now replace our old $f$ by the homotopic $f_{1}$.

By compactness of $D^{m}$, we can refine the cover by the $U_{a}$ to a cover by finitely many contractible sets $V_{1}, \ldots, V_{r} \subseteq D^{m}$, with corresponding regular values $a_{i} \in \mathbb{R}$. After possibly perturbing the $a_{i}$, we can assume they are different. We may choose an $\varepsilon>0$ such that the intervals $\left(a_{i}-2 \varepsilon, a_{i}+2 \varepsilon\right)$ are disjoint. By Lemma 3.4 we can suppose, after possibly changing $f$ by a homotopy concentrated in $\left(a_{i}-2 \varepsilon, a_{i}+2 \varepsilon\right)$, that for all $y \in V_{i}$, the element $f(y) \in \psi_{\theta}(n, k)$ is cylindrical in $x_{1}^{-1}\left(a_{i}-\varepsilon, a_{i}+\varepsilon\right)$, ie that there is an element $\lambda_{i}(y) \in \psi_{\theta_{d-1}}(n-1, k-1)$ such that the two elements

$$
f(y) \text { and }\left(\mathbb{R} \times \lambda_{i}(y)\right) \in \Psi_{\theta}\left(\mathbb{R}^{n}\right)
$$

become equal in $\Psi_{\theta}\left(x_{1}^{-1}\left(a_{i}-\varepsilon, a_{i}+\varepsilon\right)\right)$. By Proposition 3.6, $\pi_{0}\left(\psi_{\theta}(n-1, k-1)\right)=$ $\pi_{0}\left(\psi_{\theta}(n, k)\right)$, therefore the element $\lambda_{i}(y)$ must be in the basepoint component of $\psi_{\theta}(n-1, k-1)$ and since the $V_{i}$ are contractible, we can pick a smooth homotopy

$$
[0,1] \times V_{i} \stackrel{\Lambda_{i}}{\rightarrow} \psi_{\theta_{d-1}}(n-1, k-1),
$$

with $\Lambda_{i}(0,-)=\lambda_{i}$ and $\Lambda_{i}(1,-)=\varnothing$.

Pick a $\delta>0$ with $3 \delta<\varepsilon$, and a smooth function $\rho: \mathbb{R} \rightarrow[0,1]$ which is 1 on $(-\delta, \delta)$ and has support in $(-2 \delta, 2 \delta)$. Finally pick $\tau_{i}: V_{i} \rightarrow[0,1]$ with compact support and with $\bigcup_{i} \tau_{i}^{-1}(1)=D^{m}$ and define a homotopy by

$$
\begin{aligned}
V_{i} \stackrel{h_{t}^{i}}{\rightarrow} C^{\infty}\left(\mathbb{R}, \psi_{\theta_{d-1}}(n-1, k-1)\right), \quad t \in[0,1] \\
x \mapsto\left(b \mapsto \Lambda_{i}\left(t \tau_{i}(x) \rho\left(b-a_{i}\right), x\right)\right) .
\end{aligned}
$$

The homotopy starts with the map $h_{0}^{i}$ which sends all $x$ to the constant path at $\lambda_{i}(x)$. At any time $t$ the map $h_{t}^{i}(x): \mathbb{R} \rightarrow \psi_{\theta_{d-1}}(n-1, k-1)$ is constant outside of $\left(a_{i}-2 \delta, a_{i}+2 \delta\right)$. The homotopy ends at $h_{1}^{i}(x)$, which maps $\left(a_{i}-\delta, a_{i}+\delta\right)$ to the empty set. 
By taking graphs (ie composing with the function $\Gamma$ from Definition 3.2) we get a homotopy of maps

$$
\begin{aligned}
V_{i} & \rightarrow \psi_{\theta}(n, k), \quad t \in[0,1] \\
x & \mapsto \Gamma\left(h_{t}^{i}(x)\right)
\end{aligned}
$$

which at $t=0$ is $x \mapsto \mathbb{R} \times \lambda_{i}(x)$, so it agrees with $f$ on $x_{1}^{-1}\left(a_{i}-3 \delta, a_{i}+3 \delta\right)$, and at $t=1$ maps any $x \in \tau_{i}^{-1}(1)$ to an element which is empty inside $x_{1}^{-1}\left(a_{i}-\delta, a_{i}+\delta\right)$. At any time $t$, it agrees with $f$ when restricted to $x_{1}^{-1}\left(\left(a_{i}-3 \delta, a_{i}+3 \delta\right)-\left(a_{i}-2 \delta, a_{i}+2 \delta\right)\right)$ so by the sheaf property of $\Psi^{\theta}$ we can define a homotopy of maps $V_{i} \rightarrow \psi_{\theta}(n, k)$ whose restriction to $x_{1}^{-1}(\mathbb{R}-(a-2 \delta, a+2 \delta))$ is the constant homotopy of $\left.f\right|_{V_{i}}$ and whose restriction to $x_{1}^{-1}\left(a_{i}-3 \delta, a_{i}+3 \delta\right)$ is the homotopy (3-11). This homotopy is constant outside a compact subset of $V_{i}$, so it extends to a homotopy of the map $f$ which at time $t=1$ maps $\tau_{i}^{-1}(1)$ into $\psi_{\theta}^{0}(n, k)$. We have only changed $f$ inside $x_{1}^{-1}\left(a_{i}-2 \delta, a_{i}+2 \delta\right)$, so we can carry out this construction for other $a_{j}$ 's as well. In the end we have homotoped $f$ into a map to $\psi_{\theta}^{0}(n, k)$ as desired.

It remains to show that this is a relative homotopy. Suppose we have an $x \in \partial D^{m}$, so $f(x) \in \psi_{\theta}^{0}(n, k)$. Then there is a $t \in(0,1)$ that is a regular value of $x_{1}: f(x) \rightarrow \mathbb{R}$ such that $x_{1}^{-1}(t)=\varnothing$ (after we have replaced $f$ by $f_{1}$ as described in the second paragraph). If $t$ is not in $\bigsqcup_{i}\left(a_{i}-\varepsilon, a_{i}+\varepsilon\right)$, then all the homotopies we perform are constant near height $t$, so the level set at $t$ is always empty. If $t \in\left(a_{i}-\varepsilon, a_{i}+\varepsilon\right)$ then $\lambda_{i}=\varnothing$, so we can choose $\Lambda_{i}$ to be constantly $\varnothing$. The homotopy is then constant near height $t$, so the level set at $t$ is always empty and we remain in $\psi_{\theta}^{0}(n, k)$.

Proof of Theorem 3.13 We combine the three propositions to get the weak homotopy equivalences

$$
\psi_{\theta}(n, k-1) \rightarrow \Omega\left\|N_{\bullet} \psi_{\theta}(n, k-1)\right\| \rightarrow \Omega \psi_{\theta}^{0}(n, k) \rightarrow \Omega \psi_{\theta}^{\varnothing}(n, k)=\Omega \psi_{\theta}(n, k) .
$$

We will now explain how to identify the homotopy type of $\psi_{\theta}(n, n)=\Psi_{\theta}\left(\mathbb{R}^{n}\right)$.

Theorem 3.22 There is a homotopy equivalence

$$
\Psi_{\theta}\left(\mathbb{R}^{n}\right) \simeq \operatorname{Th}\left(\theta_{d, n}^{*}\left(\gamma_{d, n}^{\perp}\right) \rightarrow \mathbf{X}\left(\mathbb{R}^{n}\right)\right),
$$

where $\gamma_{d, n}^{\perp}$ is the orthogonal complement to the tautological bundle over $\operatorname{Gr}_{d}\left(\mathbb{R}^{n}\right)$.

Proof First let $\Psi_{\theta}\left(\mathbb{R}^{n}\right)^{\circ} \subseteq \Psi_{\theta}\left(\mathbb{R}^{n}\right)$ be the subspace of those $\theta$-manifolds which contain the origin. Write $L^{\theta}$ for the subspace of $\Psi_{\theta}\left(\mathbb{R}^{n}\right)^{\circ}$ consisting of linear $\theta-$ manifolds, ie those where the underlying manifold is a $d$-plane and the $\theta$-structure is constant. There is a map $\mathbf{X}\left(\mathbb{R}^{n}\right) \rightarrow L^{\theta}$ which sends a pair $(V, x)$ of a $d$-dimensional 
plane $V$ in $\mathbb{R}^{d}$ and a point $x \in \mathbf{X}$ over $i(V)$ to the pair $(V, l)$ of an element of $\mathrm{Gr}_{d}\left(\mathbb{R}^{n}\right)$ and a bundle map $l: T V=V \times V \rightarrow \theta^{*} \gamma$ given by $(v, \bar{v}) \mapsto(x, i(\bar{v}))$. This gives a map of fibrations over $\mathrm{Gr}_{d}\left(\mathbb{R}^{n}\right)$, with map on fibres over $V$ given by the inclusion $\operatorname{Fib}(\theta) \rightarrow \operatorname{Bun}\left(V, \theta^{*} \gamma\right)$. This is a homotopy equivalence (it is essentially the inclusion of the fibre of $\theta$ into its homotopy fibre, and $\theta$ has been assumed to be a Serre fibration).

Let $F_{t}: \mathbb{R}^{n} \rightarrow \mathbb{R}^{n}$ be scalar multiplication by $(1-t)$ and define a homotopy

$$
S:[0,1] \times \Psi_{\theta}\left(\mathbb{R}^{n}\right)^{\circ} \rightarrow \Psi_{\theta}\left(\mathbb{R}^{n}\right)^{\circ}
$$

as follows: on underlying manifolds let

$$
S(t, W)= \begin{cases}F_{t}^{-1}(M) & \text { if } t<1, \\ T_{0} M & \text { if } t=1 .\end{cases}
$$

To define the $\theta$-structure on $S(t, M)$ we use the map $F_{t} \times \mathrm{Id}: S(t, M) \times \mathbb{R}^{n} \rightarrow M \times \mathbb{R}^{n}$, which restricts to a fiberwise linear isomorphism

$$
T(S(t, M)) \rightarrow T M
$$

over $F_{t}$ (which is not the same as $D F_{t}$ ) and we give $S(t, M)$ the $\theta$-structure obtained by composition. This defines a continuous homotopy such that $S(0, W)=W$, $S(1, W) \in L^{\theta}$ and $S$ preserves $L^{\theta}$, so it gives a deformation retraction of $\Psi_{\theta}\left(\mathbb{R}^{n}\right)^{\circ}$ to $L^{\theta} \simeq \mathbf{X}\left(\mathbb{R}^{n}\right)$.

There is an $(n-d)$-dimensional vector bundle $v \rightarrow \Psi_{\theta}\left(\mathbb{R}^{n}\right)^{\circ}$ which at a point $W$ has fibre $v_{0} W$ the normal space to the manifold at 0 . There is a map $e: v \rightarrow \Psi_{\theta}\left(\mathbb{R}^{n}\right)$ sending $\left(W, v \in v_{0} W\right)$ to the translated manifold $W+v$. Restricted to a neighbourhood of the 0 -section in $v$, this gives an embedding onto the open subspace $U$ of $\Psi_{\theta}\left(\mathbb{R}^{n}\right)$ of those manifolds having a unique closest point to the origin. The complement $C$ of this embedding consists of manifolds which do not have a unique closest point to the origin; in particular, they do not contain it. The isotopy $\cdot(1 /(1-t)): \mathbb{R}^{n} \rightarrow \mathbb{R}^{n}, t \in[0,1)$ produces a map $H:[0,1) \times C \rightarrow C$, as it moves points on a manifold uniformly away from the origin. We can extend it to a continuous map $H:[0,1] \times C \rightarrow C$ by $H(1, c)=\varnothing$, which gives a contraction of $C$.

The map $C \rightarrow \Psi_{\theta}\left(\mathbb{R}^{n}\right)$ is a cofibration since it is a pushout of the cofibration $v \cap e^{-1}(C) \rightarrow v$, so collapsing $C$ gives a homotopy equivalent space. On the other hand, collapsing $C$ gives a space homeomorphic to that obtained by collapsing $v \cap e^{-1}(C)$ in $v$. This is the Thom space of $v$, so $\Psi_{\theta}\left(\mathbb{R}^{n}\right) \simeq \operatorname{Th}\left(v \rightarrow \psi_{\theta}(n, n)^{\circ}\right)$.

Combining Theorem 3.13 and Theorem 3.22 finishes the proof of Theorem 3.12. 


\section{Proof of the main theorems}

In this section we will prove our main results, Theorem A and Theorem B. The inclusion of a full subcategory $\mathcal{D}$ of $\mathcal{C}_{\theta}^{\bullet}$ into $\mathcal{C}_{\theta}$ will be considered in several steps. Recall that by Theorem 3.9 and Theorem 3.10, $B \mathcal{C}_{\theta} \simeq \psi_{\theta}(\infty, 1)$. We will give a similar model for $B \mathcal{D}$. We first describe some variations on the space $\psi_{\theta}(\infty, 1)$.

Recall from the introduction that we have chosen a $\theta$-structure on $\mathbb{R}^{d}$, thought of as a vector bundle over a point, and there is an induced structure on any framed manifold called the standard $\theta$-structure.

Definition 4.1 For $1>\varepsilon>0$, let $\psi_{\theta}(n, 1)^{\varepsilon} \subseteq \psi_{\theta}(n, 1)$ be the subspace where the manifold satisfies

and

$$
\begin{aligned}
& M \subseteq \mathbb{R} \times(-1,1)^{d-1} \times[0,1)^{n-d} \\
& L_{\varepsilon}=\mathbb{R} \times(-\varepsilon, \varepsilon)^{d-1} \times\{0\} \subseteq M,
\end{aligned}
$$

and that the tangential structure $l$ is standard on $L_{\varepsilon}$ with respect to the framing of $L_{\varepsilon}$ given by the vector fields $\partial / \partial x_{1}, \ldots, \partial / \partial x_{d}$. If $\varepsilon>\varepsilon^{\prime}$, there is an inclusion $\psi_{\theta}(n, 1)^{\varepsilon} \rightarrow \psi_{\theta}(n, 1)^{\varepsilon^{\prime}}$. Define $\psi_{\theta}(n, 1)^{\bullet}=\operatorname{colim}_{\varepsilon} \psi_{\theta}(n, 1)^{\varepsilon}$, with the colimit topology. There is a continuous injection $\psi_{\theta}(n, 1)^{\bullet} \rightarrow \psi_{\theta}(n, 1)$.

We define $\psi_{\theta_{d-1}}(n-1,0)^{\bullet}$ similarly, where $\psi_{\theta_{d-1}}(n-1,0)^{\varepsilon} \subseteq \psi_{\theta_{d-1}}(n-1,0)$ is the subspace of those manifolds $M$ that satisfy $M \subseteq(-1,1)^{d-1} \times[0,1)^{n-d}$ and $(-\varepsilon, \varepsilon)^{d-1} \times\{0\} \subseteq M$.

In Definition 1.1 we briefly described the objects and morphisms of a category $\mathcal{C}_{\theta}^{\bullet}$. A more precise definition is as follows.

Definition 4.2 Let $\mathcal{C}_{\theta}^{\bullet}\left(\mathbb{R}^{n}\right)$ have object space the subspace of $\psi_{\theta_{d-1}}(n-1,0)^{\bullet}$ consisting of connected manifolds. The set of nonidentity morphisms from $M_{0}$ to $M_{1}$ is the set of $(t, W) \in \mathbb{R} \times \psi_{\theta}(n, 1)^{\bullet}$ such that $t>0$, there is an $\varepsilon>0$ such that

$$
\begin{aligned}
\left.W\right|_{(-\infty, \varepsilon) \times \mathbb{R}^{n-1}} & =\left.\left(\mathbb{R} \times M_{0}\right)\right|_{(-\infty, \varepsilon) \times \mathbb{R}^{n-1}} \\
\left.W\right|_{(t-\varepsilon, \infty) \times \mathbb{R}^{n-1}} & =\left.\left(\mathbb{R} \times M_{1}\right)\right|_{(t-\varepsilon, \infty) \times \mathbb{R}^{n-1}},
\end{aligned}
$$

and such that $W \cap[0, t] \times \mathbb{R}^{n-1}$ is connected. Composition in the category is as in Definition 3.7. The total space of morphisms is topologised as a subspace of $(\{0\} \amalg(0, \infty)) \times \psi_{\theta}(n, 1)^{\bullet}$, where $(0, \infty)$ is given the usual topology. We shall be mostly concerned with the colimit $\mathcal{C}_{\theta}^{\bullet}=\operatorname{colim}_{n \rightarrow \infty} \mathcal{C}_{\theta}^{\bullet}\left(\mathbb{R}^{n}\right)$. 
Definition 4.3 Let the subspace

$$
\psi_{\theta}^{\mathrm{nc}}(n, 1)^{\bullet} \subseteq \psi_{\theta}(n, 1)^{\bullet}
$$

consist of those manifolds $W$ having no compact path components.

Definition 4.4 For $\mathbf{C}$ a collection of elements of $\psi_{\theta_{d-1}}(\infty, 0)$, let the subspace

$$
\psi_{\theta}(\infty, 1)_{\mathbf{C}} \subseteq \psi_{\theta}(\infty, 1)
$$

consist of those manifolds $W$ for which there exists a regular value $t \in(-1,1)$ of $x_{1}: W \rightarrow \mathbb{R}$ such that $W_{t}$ is in $\mathbf{C}$. Write Conn for the collection of elements of $\psi_{\theta_{d-1}}(\infty, 0)$ which are connected manifolds. Thus $\psi_{\theta}(\infty, 1)_{\text {Conn }}$ consists of those $W$ such that some $W_{t}$ is connected, for $t \in(-1,1)$.

In this definition we have insisted on regular values in $(-1,1)$ exhibiting an element as a member of the space $\psi_{\theta}(\infty, 1)_{\mathbf{C}}$. If we merely ask for such regular values in $\mathbb{R}$, we get a weakly homotopy equivalent space, but the current definition will simplify certain constructions later.

Theorem 4.5 Let $\mathbf{C}$ be a collection of objects of $\mathcal{C}_{\theta}^{\bullet}$ (so $\mathbf{C} \subseteq \mathbf{C o n n}$ ), and $\mathcal{D}$ be the full subcategory on $\mathbf{C}$. Then there is a weak homotopy equivalence

$$
B \mathcal{D} \simeq \psi_{\theta}^{\mathrm{nc}}(\infty, 1)_{\mathbf{C}}^{\bullet}
$$

Proof This is exactly as the proof of Theorem 3.9. Note that if $W \in \psi_{\theta}^{\mathrm{nc}}(\infty, 1)^{\bullet}$ and $a_{0}$ and $a_{1}$ are two regular values of $x_{1}: W \rightarrow \mathbb{R}$ such that $W_{a_{v}} \in \mathbf{C}$, then the fact that elements of $\mathbf{C}$ are connected and $W$ is noncompact implies that the manifold $W \cap x_{1}^{-1}\left(\left[a_{0}, a_{1}\right]\right)$ is also connected. The analogous $D_{\theta}\left(\mathbb{R}^{\infty}\right)_{\mathbf{C}}^{\bullet}$ is then the topological poset consisting of pairs $(t, W) \in \mathbb{R} \times \psi_{\theta}^{\text {nc }}(\infty, 1)^{\bullet}$ with $t$ regular for $x_{1}: W \rightarrow \mathbb{R}$ and $W_{t} \in \mathbf{C}$. This allows one to mimic the proof of Theorem 3.9.

However the space $\psi_{\theta}^{\mathrm{nc}}(\infty, 1)_{\mathbf{C}}^{\bullet}$ has such regular values in $(-1,1)$, not merely in $\mathbb{R}$. Thus it does not have a map from the poset $D_{\theta}\left(\mathbb{R}^{\infty}\right)_{\mathbf{C}}^{\bullet}$, but only from the full subposet $\mathcal{P} \subseteq D_{\theta}\left(\mathbb{R}^{\infty}\right)_{\mathrm{C}}^{\bullet}$ of pairs $(t, W)$ with $t \in(-1,1)$. We must show that the inclusion of this subposet gives an equivalence on classifying spaces. This is so as it induces a levelwise homotopy equivalence on simplicial nerves: a homotopy inverse to the inclusion $N_{k} \mathcal{P} \rightarrow N_{k} D_{\theta}\left(\mathbb{R}^{\infty}\right)_{\mathbf{C}}^{\bullet}$ is giving by affine scaling in the $\mathbb{R}$ direction until all regular values lie in the interval $(-1,1)$.

As in the proof of Theorem 3.10 the fibre of $\mathcal{P} \rightarrow \psi_{\theta}^{\mathrm{nc}}(\infty, 1)_{\mathbf{C}}^{\bullet}$ over $W$ becomes the simplex with vertices all possible choices of $a \in(-1,1)$ with $W_{a} \in \mathbf{C}$, which is contractible. 
To finish the proof of Theorem A we need to prove that the inclusion

$$
\psi_{\theta}^{\mathrm{nc}}(\infty, 1)_{\mathbf{C o n n}}^{\bullet} \rightarrow \psi_{\theta}(\infty, 1)
$$

is a weak equivalence. This will be done in the rest of this section, and will be broken up into several steps that we treat separately. The inclusion (4-1) factors as

$$
\psi_{\theta}^{\mathrm{nc}}(\infty, 1)_{\text {Conn }}^{\bullet} \rightarrow \psi_{\theta}^{\mathrm{nc}}(\infty, 1)^{\bullet} \rightarrow \psi_{\theta}(\infty, 1)^{\bullet} \rightarrow \psi_{\theta}(\infty, 1) .
$$

Starting from the right, we prove in Lemma 4.6 below that the inclusion $\psi_{\theta}(n, 1)^{\bullet} \rightarrow$ $\psi_{\theta}(n, 1)$ is a homotopy equivalence by giving two explicit maps that are homotopy inverse. Then in Lemma 4.7 we show that $\psi_{\theta}^{\mathrm{nc}}(n, 1)^{\bullet} \rightarrow \psi_{\theta}(n, 1)^{\bullet}$ is a weak homotopy equivalence. These steps are both fairly easy. In Section 4.2 we prove that $\psi_{\theta}^{\mathrm{nc}}(\infty, 1)_{\text {Conn }}^{\bullet} \rightarrow \psi_{\theta}^{\mathrm{nc}}(\infty, 1)^{\bullet}$ is a weak equivalence. For the proof we use 0 -surgery to make objects connected, but in order to get a homotopy equivalence we need a fairly elaborate procedure for doing 0 -surgeries in families.

Lemma 4.6 The inclusion $i: \psi_{\theta}(n, 1)^{\bullet} \rightarrow \psi_{\theta}(n, 1)$ is a weak homotopy equivalence.

Proof For $X$ a compact space, any map $X \rightarrow \psi_{\theta}(n, 1)^{\bullet}$ factors through some $\psi_{\theta}(n, 1)^{\varepsilon}$ by a standard property of the colimit topology. Thus it is enough to show that $\psi_{\theta}(n, 1)^{\varepsilon} \rightarrow \psi_{\theta}(n, 1)$ is a weak homotopy equivalence.

We can define a product $\amalg: \psi_{\theta}(n, 1) \times \psi_{\theta}(n, 1) \rightarrow \psi_{\theta}(n, 1)$ as follows. The manifold $W_{1} \amalg W_{2}$ is obtained by taking the union of the disjoint manifolds $W_{1}$ and $W_{2}+e_{d+1}$ and scaling the $(d+1)$-st coordinate by $1 / 2$.

Now pick a "cylinder" $W_{0} \in \psi_{\theta}(n, 1)^{\varepsilon}$ and define a map

$$
\begin{aligned}
c: \psi_{\theta}(n, 1) & \rightarrow \psi_{\theta}(n, 1)^{\varepsilon} \\
M & \mapsto W_{0} \amalg M .
\end{aligned}
$$

Note that $W_{0} \amalg M$ does lie in $\psi_{\theta}(n, 1)^{\varepsilon}$ by construction of the product. Then the composition

$$
\psi_{\theta}(n, 1) \stackrel{c}{\rightarrow} \psi_{\theta}(n, 1)^{\varepsilon} \stackrel{i}{\rightarrow} \psi_{\theta}(n, 1)
$$

is $M \mapsto i\left(W_{0}\right) \amalg M$, which is homotopic to the identity map if we pick $W_{0}$ in the component of the basepoint. The effect of the reverse composition

$$
\psi_{\theta}(n, 1)^{\varepsilon} \stackrel{i}{\rightarrow} \psi_{\theta}(n, 1) \stackrel{c}{\rightarrow} \psi_{\theta}(n, 1)^{\varepsilon}
$$

is shown in Figure 3. and the homotopy to the identity map is as in Figure 4. 

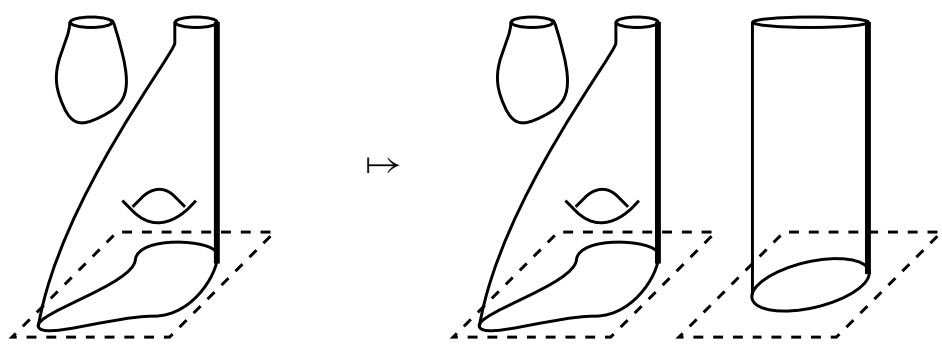

Figure 3

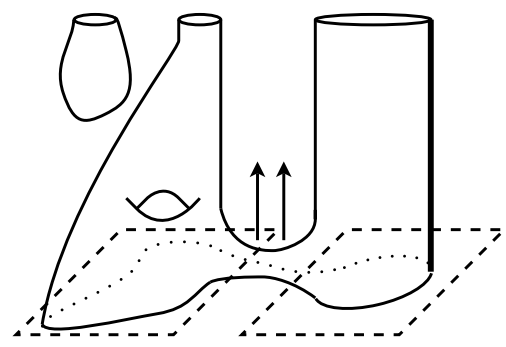

Figure 4

Lemma 4.7 The inclusion $\psi_{\theta}^{\mathrm{nc}}(n, 1)^{\bullet} \rightarrow \psi_{\theta}(n, 1)^{\bullet}$ is a weak homotopy equivalence.

Proof Again we will show that the relative homotopy groups vanish. Let

$$
f:\left(D^{m}, \partial D^{m}\right) \rightarrow\left(\psi_{\theta}(n, 1)^{\bullet}, \psi_{\theta}^{\mathrm{nc}}(n, 1)^{\bullet}\right)
$$

be a smooth map. We will show it is relatively homotopic to a map into $\psi_{\theta}^{\mathrm{nc}}(n, 1)^{\bullet}$.

Consider the height function $x_{1}: \Gamma(f) \rightarrow \mathbb{R}$. After perturbing $f$, we may assume that for all $x \in D^{m}$, no path component of $f(x)$ is contained in $x_{1}^{-1}(0)$. For each $x \in X$, we can choose an $\varepsilon_{x}>0$ such that no path component of $f(x)$ is contained in $f(x) \cap x_{1}^{-1}\left[-\varepsilon_{x}, \varepsilon_{x}\right]$. There is an open neighbourhood $U_{x} \ni x$ for which this is still true.

The $\left\{U_{x}\right\}_{x \in X}$ form an open cover of $X$ : take a finite subcover $\left\{U_{i}\right\}$, and let $\varepsilon=$ $\min \left(\varepsilon_{i}\right)$. Then there is no path component of $f(x)$ in $x_{1}^{-1}[-\varepsilon, \varepsilon]$ for any $x \in X$. Choosing an isotopy of embeddings from the identity on $\mathbb{R}$ to a diffeomorphism that takes $\mathbb{R}$ to $(-\varepsilon, \varepsilon)$ defines a relative homotopy of the map $f$ into the subspace $\psi_{\theta}^{\mathrm{nc}}(n, 1)^{\bullet}$.

Our aim is to show that the inclusion

$$
\psi_{\theta}^{\mathrm{nc}}(\infty, 1)_{\text {Conn }}^{\bullet} \psi_{\theta}^{\mathrm{nc}}(\infty, 1)^{\bullet}
$$


is a weak homotopy equivalence, for any dimension $d \geq 2$ and for all tangential structures $\theta$ such that $\mathbf{X}$ is connected and $S^{d}$ admits a $\theta$-structure. The idea for doing so is relatively simple, and proceeds by showing the relative homotopy groups $\pi_{k}\left(\psi_{\theta}^{\mathrm{nc}}(\infty, 1)_{\mathbf{C o n n}}^{\bullet}, \psi_{\theta}^{\mathrm{nc}}(\infty, 1)^{\bullet}\right)$ are trivial.

It is instructive to first consider the case $k=0$. Suppose we are given a $W \in \psi_{\theta}^{\text {nc }}(\infty, 1)^{\bullet}$. We wish to produce a $\bar{W} \in \psi_{\theta}^{\mathrm{nc}}(\infty, 1)^{\bullet}$ Conn $^{\circ}$ and a path from $W$ to $\bar{W}$ in $\psi_{\theta}^{\mathrm{nc}}(\infty, 1)^{\bullet}$. The condition to belong to $\psi_{\theta}^{\text {nc }}(\infty, 1)^{\bullet}$ conn is the existence of a level set which is connected, so let us choose a regular value $a_{0} \in(-1,1)$ of $x_{1}: W \rightarrow \mathbb{R}$ and write $W_{a_{0}}=W \cap x_{1}^{-1}\left(a_{0}\right)$ for the level set. In general $W_{a_{0}}$ will not be connected, so we intend to obtain $\bar{W}$ by performing 0 -surgery on $W$ near $a_{0}$, so as to make the level set at $a_{0}$ connected: the result $\bar{W}$ of this surgery will lie in $\psi_{\theta}^{\mathrm{nc}}(\infty, 1)^{\bullet}$ Conn .

To carry out 0 -surgery on an element of $\psi_{\theta}(\infty, 1)$ we need to explain how to give a height function and a $\theta$-structure on the handles we attach. We do this in the following section.

\subsection{Parametrised 0-surgery}

Recall that if $W$ is an (abstract) smooth $d$-manifold and $e: S^{0} \times D^{d} \rightarrow W$ is an embedding, then performing 0 -surgery on $W$ along $e$ amounts to removing $e\left(S^{0} \times \operatorname{int}\left(D^{d}\right)\right)$ from $W$ and gluing in $D^{1} \times S^{d-1}$ along $S^{0} \times S^{d-1}$. In our setup, $W \in \psi^{\theta}(\infty, 1)$ has more structure, the essential parts of which are the height function $x_{1}: W \rightarrow \mathbb{R}$ and the $\theta$-structure $l: T W \rightarrow \theta^{*} \gamma$. We explain how to extend these to the manifold $\bar{W}$ resulting from performing surgery.

4.1.1 $\theta$-structures The following depends critically on our assumptions on $\theta: \mathbf{X} \rightarrow$ $B O(d)$, namely that $\mathbf{X}$ be path connected and that $S^{d}$ admit a $\theta$-structure.

Proposition 4.8 Let $W$ be a smooth $d$-manifold that is equipped with a $\theta$-structure $l: T W \rightarrow \theta^{*} \gamma$ and let

$$
e: S^{0} \times D^{d} \rightarrow W
$$

be an embedding. Let $\mathcal{K}^{e}(W)$ denote the result of performing surgery on $W$ along $e$. After possibly changing $e$ by a self-diffeomorphism of $D^{d}$, the $\theta$-structure on the submanifold

$$
W-e\left(S^{0} \times \operatorname{int} D^{d}\right) \subseteq \mathcal{K}^{e}(W)
$$

extends to a $\theta$-structure on $\mathcal{K}^{e}(W)$. 
Proof After cutting out $e\left(S^{0} \times \operatorname{int} D^{d}\right) \subseteq W$ and gluing in the $D^{1} \times S^{d-1}$, the $\theta$-structure on $W$ gives a bundle map

$$
\left.T\left(D^{1} \times S^{d-1}\right)\right|_{\partial D^{1} \times S^{d-1}} \rightarrow \theta^{*} \gamma,
$$

and the problem becomes to extend this to a bundle map over all of $D^{1} \times S^{d-1}$. This obstruction theoretic question only depends on the homotopy class of the $\theta$-structure on $W$ over each sphere $e\left(\{ \pm 1\} \times \partial D^{d}\right)$.

The space of $\theta$-structures on $D^{d}$ is homotopy equivalent to the fibre of the fibration $\theta: \mathbf{X} \rightarrow B O(d)$. The assumption that $\mathbf{X}$ be path connected implies that $\pi_{1} B O(d)$ acts transitively on this fibre, so up to possibly changing the sign of a coordinate in $D^{d}$, all $\theta$-structures on it are homotopic. In particular we can arrange that after possibly composing the embedding (4-2) with a self-diffeomorphism of $S^{0} \times D^{d}$, the $\theta$-structures on $D^{d}$ induced by the embeddings $e( \pm 1,-): D^{d} \rightarrow W$ are homotopic to the $\theta$-structures on the disks

$$
D_{ \pm}^{d}=\left\{x \in S^{d} \mid \pm x_{1} \geq \frac{1}{2}\right\}
$$

with respect to some $\theta$-structure $l_{0}: T S^{d} \rightarrow \theta^{*} \gamma$ on $S^{d}$, chosen once and for all. (Our assumption on $\theta: X \rightarrow B O(d)$ is precisely that such a choice can be made.) But then the bundle map (4-3) extends over the cylinder $D^{1} \times S^{d-1}$, since that cylinder can be identified with

$$
\left\{x \in S^{d}|| x_{1} \mid \leq \frac{1}{2}\right\} .
$$

4.1.2 Height functions Pick once and for all a smooth family of functions

$$
\lambda_{w}:[0,1] \rightarrow[w, 1], \quad w \in\left[0, \frac{1}{2}\right]
$$

such that $\lambda(t)=t$ on $\lambda^{-1}\left(\left[\frac{3}{4}, 1\right]\right)$, that $\lambda^{-1}(w)=\left[0, \frac{1}{2}\right]$, and such that $\lambda^{\prime}(t)>0$ on $\lambda^{-1}(w, 1)$. Define a family of smooth functions $\varphi_{w}$ by

$$
\begin{aligned}
D^{1} \times S^{d-1} & \stackrel{\varphi_{w}}{\rightarrow} D^{d}, \quad w \in\left[0, \frac{1}{2}\right] \\
(t, x) & \mapsto \lambda_{w}(|t|) x .
\end{aligned}
$$

The domain $D^{1} \times S^{d-1}$ is the disjoint union of the open sets $U^{+}=\left(\frac{1}{2}, 1\right] \times S^{d-1}$ and $U^{-}=\left[-1,-\frac{1}{2}\right) \times S^{d-1}$, and the closed set $C=\left[-\frac{1}{2}, \frac{1}{2}\right] \times S^{d-1}$. Then the map $\varphi_{w}$ restricts to diffeomorphisms

$$
\begin{aligned}
& U^{+} \stackrel{\left.\varphi_{w}\right|_{U^{+}}}{\longrightarrow} D^{d}-w D^{d} \\
& U^{-} \stackrel{\left.\varphi_{w}\right|_{U^{-}}}{\longrightarrow} D^{d}-w D^{d}
\end{aligned}
$$


and the restriction to $C=\left[-\frac{1}{2}, \frac{1}{2}\right] \times S^{d-1}$ can be factored as

$$
C \stackrel{\text { proj }}{\rightarrow} S^{d-1} \stackrel{w}{\rightarrow} \partial\left(w D^{d}\right),
$$

where the second map $w: S^{d-1} \rightarrow \partial\left(w D^{d}\right)$ denotes multiplication by $w$ in $\mathbb{R}^{n}$.

Let $W$ be a smooth $d$-manifold with a smooth height function $x_{1}: W \rightarrow \mathbb{R}$. If $e: S^{0} \times D^{d} \rightarrow W$ and $w \in\left[0, \frac{1}{2}\right]$ is such that $x_{1} \circ e(+1, x)=x_{1} \circ e(-1, x)$ for $|x| \leq w$ we define a map $D^{1} \times S^{d-1} \rightarrow \mathbb{R}$ by

$$
(t, x) \mapsto \begin{cases}x_{1} \circ e\left(+1, \varphi_{w}(t, x)\right) & \text { if } t>-\frac{1}{2}, \\ x_{1} \circ e\left(-1, \varphi_{w}(t, x)\right) & \text { if } t<\frac{1}{2} .\end{cases}
$$

This is well defined because of the factorisation (4-5) (so $\varphi_{w}(t, x)$ is independent of $t$ for $\left.t \in\left[-\frac{1}{2}, \frac{1}{2}\right]\right)$.

The inverses of the diffeomorphisms (4-4) give an embedding

$$
\begin{aligned}
S^{0} \times\left(D^{d}-w D^{d}\right) & \stackrel{h_{w}}{\longrightarrow} D^{1} \times S^{d-1} \\
( \pm 1, x) & \mapsto\left(\left.\varphi_{w}\right|_{U^{ \pm}}\right)^{-1}(x)
\end{aligned}
$$

whose image is the complement of $C$. The restriction of $h_{w}$ to $h: S^{0} \times\left(D^{d}-\frac{3}{4} D^{d}\right) \rightarrow$ $D^{1} \times S^{d-1}$ is independent of the parameter $w$.

Definition 4.9 Let $W \in \psi^{\theta}(n, 1)$, let $w \in\left[0, \frac{1}{2}\right]$, and let $e: S^{0} \times D^{d} \rightarrow W$ be an embedding satisfying

$$
x_{1} \circ e(+1, x)=x_{1} \circ e(-1, x) \text { when }|x| \leq w .
$$

(i) Let $\mathcal{K}_{w}^{e}(W)$ be the smooth manifold obtained by gluing $D^{1} \times S^{d-1}$ into $W-$ $e\left(S^{0} \times w D^{d}\right)$ along the embedding (4-7). Let

$$
x_{1}: \mathcal{K}_{w}^{e}(W) \rightarrow \mathbb{R}
$$

be the function which agrees with (4-6) on $D^{1} \times S^{d-1}$ and with the old $x_{1}$ on $W-e\left(S^{0} \times w D^{d}\right)$.

(ii) Pick functions $x_{2}, x_{3}, \ldots: \mathcal{K}_{w}^{e}(W) \rightarrow(-1,1)$ extending the coordinate functions on $W-e\left(S^{0} \times D^{d}\right)$ such that the resulting map $x: \mathcal{K}_{w}^{e}(W) \rightarrow \mathbb{R}^{\infty}$ is an embedding. Also pick, by Proposition 4.8, an extension of the $\theta$-structure on $W-e\left(S^{0} \times w D^{d}\right)$ to a $\theta$ structure on $\mathcal{K}_{w}^{e}(W)$ (after possibly changing sign of a coordinate on $\left.D^{d}\right)$. We use the same notation $\mathcal{K}_{w}^{e}(W)$ for the resulting element

$$
\mathcal{K}_{w}^{e}(W) \in \psi^{\theta}(\infty, 1)
$$


In the second part of Definition 4.9, the notation $\mathcal{K}_{w}^{e}(W) \in \psi^{\theta}(\infty, 1)$ is slightly imprecise, because the element $\mathcal{K}_{w}^{e}(W)$ depends on more data than just $W, w$ and $e$. The remaining data (namely the extension of the embedding and the $\theta$-structure) will not play any role in the applications of the construction, so we omit it from the notation. The main properties of $\mathcal{K}_{w}^{e}(W)$ are recorded in the following two lemmas.

Lemma 4.10 Let $W, w$ and $e$ be as in Definition 4.9 and let

$$
[a, b]=x_{1} \circ e\left(+1, w D^{d}\right)=x_{1} \circ e\left(-1, w D^{d}\right) .
$$

Then the level sets $W \cap x_{1}^{-1}(t)$ and $\mathcal{K}_{w}^{e}(W) \cap x_{1}^{-1}(t)$ are canonically diffeomorphic, for all $t \in \mathbb{R}-[a, b]$.

In particular in the extreme case $w=0$, level sets agree except at the level $a=$ $x_{1} \circ e(+1,0)=x_{1} \circ e(-1,0)$.

Proof The manifold $\mathcal{K}_{w}^{e}(W)$ is the disjoint union of the open set $W-e\left(S^{0} \times w D^{d}\right)$ and the closed set $C=\left[-\frac{1}{2}, \frac{1}{2}\right] \times S^{d-1}$. The lemma follows because $x_{1}: W \rightarrow \mathbb{R}$ takes the values in $[a, b]$ on $e\left(S^{0} \times w D^{d}\right) \subseteq W$ and (4-7) takes values in $[a, b]$ on $C \subseteq \mathcal{K}_{w}^{e}(W)$. Outside these closed sets, $W$ and $\mathcal{K}_{w}^{e}(W)$ are canonically diffeomorphic by a level-preserving diffeomorphism.

The next lemma is concerned with what happens to the level sets that change, in some cases. More precisely we will consider $w>0$ and embeddings $e: S^{0} \times D^{d} \rightarrow W$ that are height preserving, ie such that

$$
x_{1} \circ e( \pm 1, y)=a+y_{1} \quad \text { for }|y| \leq w,
$$

where $a=x_{1} \circ e(+1,0)=x_{1} \circ e(-1,0)$ and $y_{1}$ is the first coordinate of $y \in D^{d} \subseteq \mathbb{R}^{d}$. In that case $e$ restricts to an embedding

$$
S^{0} \times\left(w D_{0}^{d}\right) \rightarrow W_{a}=W \cap x_{1}^{-1}(a),
$$

where $D_{0}^{d}=D^{d} \cap x_{1}^{-1}(0) \cong D^{d-1}$.

Lemma 4.11 Let $w>0$ and $e: S^{0} \times D^{d} \rightarrow W$ be as in (4-8), and assume $a$ is a regular value of $x_{1}: W \rightarrow \mathbb{R}$. Then the level set $\mathcal{K}_{w}^{e}(W) \cap x_{1}^{-1}(a)$ is diffeomorphic to the manifold obtained by performing 0 -surgery on $W_{a}=W \cap x_{1}^{-1}(a)$ along the embedding

$$
S^{0} \times D^{d-1} \stackrel{\cong}{\longrightarrow} S^{0} \times\left(w D_{0}^{d}\right) \stackrel{e}{\longrightarrow} W_{a}
$$


Proof $\mathcal{K}_{w}^{e}(W)$ is obtained from $W$ by removing $e\left(S^{0} \times \operatorname{int}\left(w D^{d}\right)\right)$ and gluing in $C=$ $\left[-\frac{1}{2}, \frac{1}{2}\right] \times S^{d-1}$. On the level set at $a$, this removes the open set $e\left(S^{0} \times \operatorname{int}\left(w D_{0}^{d}\right)\right) \cong$ $S^{0} \times D^{d-1}$ and glues in the set

$$
C \cap\left(x_{1}\right)^{-1}(a)=\left[-\frac{1}{2}, \frac{1}{2}\right] \times S^{d-2},
$$

where $S^{d-2} \subseteq S^{d-1}$ is the equator.

Finally, two extended versions of the construction of $\mathcal{K}_{w}^{e}(W)$ from $W, w, e$. First a version with multiple surgeries at once.

Definition 4.12 Let $W \in \psi^{\theta}(\infty, 1)$ and $w \in\left[0, \frac{1}{2}\right]$. Let $\Lambda=\left\{\lambda_{1}, \ldots, \lambda_{r}\right\}$ be a finite set and $e: \Lambda \times S^{0} \times D^{d} \rightarrow W$ an embedding such that

$$
x_{1} \circ e\left(\lambda_{i},+1, x\right)=x_{1} \circ e\left(\lambda_{i},-1, x\right) \quad \text { when }|x| \leq w .
$$

for all $\lambda_{i} \in \Lambda$. Then define

$$
\mathcal{K}_{w}^{e}(W)=\mathcal{K}_{w}^{e\left(\lambda_{1},-\right)} \circ \cdots \circ \mathcal{K}_{w}^{e\left(\lambda_{r},-\right)}(W) \in \psi^{\theta}(\infty, 1) .
$$

This is well defined because the embeddings $e\left(\lambda_{i},-\right): S^{0} \times D^{d} \rightarrow W$ are disjoint.

Of course, the properties of levelwise surgery from Lemma 4.10 and Lemma 4.11 imply similar properties for multiple levelwise surgery. Secondly we need a parametrised version of this construction.

Proposition 4.13 Let $V$ be a contractible manifold, and $f: V \rightarrow \psi^{\theta}(n, 1)$ and $w: V \rightarrow\left[0, \frac{1}{2}\right]$ smooth maps. Let $\Lambda$ be a finite set and $e: V \times \Lambda \times S^{0} \times D^{d} \rightarrow \Gamma\left(\left.f\right|_{V}\right)$ be an embedding over $V$. Assume that the triple $(f(x), w(x), e(x))$ satisfies the assumption of Definition 4.12 for all $x \in V$. Then there is a smooth map

$$
\begin{aligned}
V & \rightarrow \psi^{\theta}(\infty, 1) \\
x & \mapsto \mathcal{K}_{w(x)}^{e(x)}(f(x)),
\end{aligned}
$$

which for each $x$ is constructed as in Definition 4.12.

Proof sketch The manifold $f(x)$ depends smoothly on $x$. If we pick the remaining coordinate functions $x_{2}, x_{3}, \ldots: \mathcal{K}_{w(x)}^{e(x)}(f(x)) \rightarrow \mathbb{R}$ in a smooth fashion, the manifold $\mathcal{K}_{w(x)}^{e(x)}(f(x))$ will also depend smoothly on $x$. Under the assumption that $V$ is contractible, the obstructions to extending the $\theta$-structure are the same as in the case where $V$ is a point.

Finally, let us remark that the surgery on elements of $\psi_{\theta}(\infty, 1)$ constructed in this section preserves the subspace $\psi_{\theta}^{\mathrm{nc}}(\infty, 1)$. It also preserves the subspace $\psi_{\theta}^{\mathrm{nc}}(\infty, 1)^{\bullet}$, provided the surgery data is disjoint from some strip $L_{\varepsilon} \subseteq W$. 


\subsection{Proof of Theorem A}

We now prove the main result of this section and finish the proof of Theorem A. Throughout this section we will assume that $d \geq 2$.

Theorem 4.14 The relative homotopy groups

$$
\pi_{k}\left(\psi_{\theta}^{\mathrm{nc}}(\infty, 1)^{\bullet}, \psi_{\theta}^{\mathrm{nc}}(\infty, 1)_{\text {Conn }}^{\bullet}\right)
$$

vanish for all $k$.

To prove this theorem we need to construct a null homotopy of an arbitrary continuous map of pairs

$$
\left(D^{k}, \partial D^{k}\right) \stackrel{f}{\rightarrow}\left(\psi_{\theta}^{\mathrm{nc}}(\infty, 1)^{\bullet}, \psi_{\theta}^{\mathrm{nc}}(\infty, 1)_{\text {Conn }}^{\bullet}\right) .
$$

The null homotopy will be constructed using the parametrised surgery developed in the previous section. We first give the local construction. It is convenient to have a word for the following property.

Definition 4.15 Let us say that an element $W \in \psi_{\theta}^{\mathrm{nc}}(n, 1)^{\bullet}$ is connected at level a if $a$ is a regular value of $x_{1}: W \rightarrow \mathbb{R}$ and if the level set $W_{a}=W \cap x_{1}^{-1}(a)$ is path connected. Thus $W \in \psi_{\theta}^{\mathrm{nc}}(n, 1)_{\text {Conn }}^{\bullet}$ if and only if it is connected at level $a$ for some $a \in(-1,1)$.

In the following, we will very often use the following construction. Let $j: \mathbb{R} \rightarrow \mathbb{R}$ be an embedding which is isotopic through embeddings to the identity, has image $(-10,10)$, and has $j(t)=t$ for $t \in[-1,1]$. Then let $s$ be the continuous map

$$
\begin{aligned}
\psi_{\theta}(n, 1) & \stackrel{s}{\rightarrow} \psi_{\theta}(n, 1) \\
M & \mapsto(j \times \mathrm{Id})^{-1}(M) .
\end{aligned}
$$

This "stretching" map will be used throughout this section.

Proposition 4.16 Let $V$ be a contractible space and $f: V \rightarrow \psi_{\theta}^{\text {nc }}(\infty, 1)^{\bullet}$ be a smooth map. Let $a \in(-1,1)$ be a regular value of $x_{1}: f(x) \rightarrow \mathbb{R}$ for all $x \in V$. Finally, let $\Lambda=\left\{\lambda_{1}, \ldots, \lambda_{r}\right\}$ be a finite set, and

$$
p: V \times \Lambda \times[0,1] \rightarrow \Gamma\left(\left.f\right|_{V}\right)
$$

be an embedding over $V$, such that: 
(a) The path $p(x, \lambda,-):[0,1] \rightarrow f(x)$ ends somewhere in $f(x) \cap x_{1}^{-1}(a)$, outside the path component of the basepoint; each nonbasepoint component contains exactly one of the ending points $p(x, \lambda, 1)$.

(b) The path $p(x, \lambda,-):[0,1] \rightarrow f(x)$ starts somewhere in $f(x)-x_{1}^{-1}([-10,10])$.

Then there is a homotopy $F:[0,1] \times V \rightarrow \psi_{\theta}^{\mathrm{nc}}(\infty, 1)^{\bullet}$, such that:

(i) $F(0,-)$ agrees with the composition

$$
V \stackrel{f}{\rightarrow} \psi_{\theta}^{\mathrm{nc}}(\infty, 1)^{\bullet} \stackrel{s}{\rightarrow} \psi_{\theta}^{\mathrm{nc}}(\infty, 1)^{\bullet} .
$$

(ii) $F(1, x)$ is connected at level $a$ for all $x \in V$. In particular,

$$
F(\{1\} \times V) \subseteq \psi_{\theta}^{\mathrm{nc}}(\infty, 1)_{\text {Conn }}^{\bullet}
$$

(iii) If $f(x) \in \psi_{\theta}^{\mathrm{nc}}(\infty, 1)_{\text {Conn }}^{\bullet}$ then $F(t, x) \in \psi_{\theta}^{\mathrm{nc}}(\infty, 1)_{\text {Conn }}^{\bullet}$ for all $t$.

Proof First construct $e:[0,1] \times V \times \Lambda \times S^{0} \rightarrow \Gamma\left(\left.f\right|_{V}\right)$ an embedding over $V$, in the following way. Recall that we have a trivialised $L_{\varepsilon}=\mathbb{R} \times(-\varepsilon, \varepsilon)^{d-1} \times\{0\} \subseteq f(x)$ for all $x$. Then pick points $x_{\lambda} \in(0, \varepsilon)^{d-1} \times\{0\}$ arbitrarily, but with all $x_{\lambda}$ different (this is possible because $d-1>0$ ). Then set

$$
\begin{aligned}
& e(t, x, \lambda,+1)=p(x, \lambda, t) \\
& e(t, x, \lambda,-1)=\left(x_{1} \circ p(x, \lambda, t), x_{\lambda}\right) .
\end{aligned}
$$

Note that $e(t, x,-)$ embeds $\Lambda \times S^{0}$ into a single level set of $x_{1}: f(x) \rightarrow \mathbb{R}$.

Thicken each embedding $e(t, x,-): \Lambda \times S^{0} \rightarrow f(x)$ to an embedding of $\Lambda \times S^{0} \times D^{d}$. We denote the resulting map by the same letter

$$
e:[0,1] \times V \times \Lambda \times S^{0} \times D^{d} \rightarrow \Gamma\left(\left.f\right|_{V}\right) .
$$

We can arrange that at $t=1$ we have the analogue of (4-8), namely

$$
x_{1} \circ e(1, x, \lambda, \pm 1, v)=a+v_{1} \quad \text { for }|v| \leq w_{0}
$$

for some $w_{0}>0$.

Let $\rho:[0,1] \rightarrow[0,1]$ be a smooth function with $\left[0, \frac{1}{2}\right] \subseteq \rho^{-1}(0)$ and $\rho(1)=1$. Set $w(t)=\rho(t) w_{0}$. Also pick a function $\tau:[0,1] \rightarrow[0,1]$ with $\tau(0)=0$ and $\left[\frac{1}{2}, 1\right] \subseteq$ $\tau^{-1}(1)$. By Proposition 4.13 we get a smooth homotopy

$$
\begin{aligned}
{[0,1] \times V } & \rightarrow \psi_{\theta}^{\mathrm{nc}}(\infty, 1) \\
(t, x) & \mapsto \mathcal{K}_{w(t)}^{e(\tau(t), x)}(f(x)) .
\end{aligned}
$$


By Lemma 4.11 this homotopy at time 1 performs 0 -surgery on the level set $x_{1}^{-1}(a)$. This 0 -surgery forms the connected sum of the basepoint component with all the other components, so the result satisfies (ii). At time 0 , we have performed 0 -surgery on points outside of $x_{1}^{-1}([-10,10])$, so we have not changed anything inside that interval. Therefore we can let $F:[0,1] \times V \rightarrow \psi_{\theta}^{\text {nc }}(\infty, 1)^{\bullet}$ be the composition of (4-12) with the stretching map $s$ from (4-11), and then $F$ satisfies (i) and (ii).

Finally, (iii) follows for any pair $(t, x)$ by cases. Either $\rho(t)=0$, so Lemma 4.10 implies there is at most one level set of $F(t, x)$ in $(-1,1)$ different from its corresponding one in $f(x)$, but if $f(x) \in \psi_{\theta}^{\mathrm{nc}}(\infty, 1)_{\text {Conn }}^{\bullet}$ then there is a small open interval of regular values in $(-1,1)$ having connected level sets, so $F(t, x)$ must still have a connected level set. Otherwise $\tau(t)=1$, so $F(t, x)$ is obtained from $f(x)$ by performing $0-$ surgery on the level set $x_{1}^{-1}(a) \cap f(x)$ to make it connected, so $F(t, x)$ has a connected level set.

Our strategy for proving vanishing of the relative homotopy groups (4-10) is to apply Proposition 4.16 locally to construct a null homotopy. More precisely we will use the following corollary of Proposition 4.16 (and its proof).

Corollary 4.17 Let $f: X \rightarrow \psi_{\theta}^{\mathrm{nc}}(\infty, 1)^{\bullet}$ be smooth. Assume that there exists an open cover $X=V_{1} \cup \cdots \cup V_{r}$ by contractible open sets $V_{i} \subseteq X$ and different $a_{i} \in(-1,1)$ such that $a_{i}$ is a regular value of $x_{1}: f(x) \rightarrow \mathbb{R}$ for all $x \in V_{i}$. Finally, assume there are finite sets $\Lambda_{i}$ and an embedding

$$
p_{i}: V_{i} \times \Lambda_{i} \times[0,1] \rightarrow \Gamma\left(\left.f\right|_{V_{i}}\right)
$$

over $V_{i}$, such that:

(a) $\left(V_{i},\left.f\right|_{V_{i}}, a_{i}, \Lambda_{i}, p_{i}\right)$ satisfy the assumptions of Proposition 4.16.

(b) For $i \neq j$, the images of $p_{i}(x,-)$ and $p_{j}(x,-)$, if both defined, are disjoint.

Then given any collection $\left\{U_{i} \subseteq V_{i}\right\}$ with $\bar{U}_{i} \subseteq V_{i}$, there is a homotopy $H:[0,1] \times X \rightarrow$ $\psi_{\theta}^{\mathrm{nc}}(\infty, 1)^{\bullet}$ such that:

(i) $H(0,-)$ agrees with the composition

$$
X \stackrel{f}{\rightarrow} \psi_{\theta}^{\mathrm{nc}}(\infty, 1)^{\bullet} \stackrel{s}{\rightarrow} \psi_{\theta}^{\mathrm{nc}}(\infty, 1)^{\bullet} .
$$

(ii) $H(1, x)$ is connected at level $a_{i}$ for all $x \in U_{i}$. In particular if the $U_{i}$ cover $X$, then

$$
H(\{1\} \times X) \subseteq \psi_{\theta}^{\mathrm{nc}}(\infty, 1)_{\text {Conn }}^{\bullet}
$$

(iii) If $f(x) \in \psi_{\theta}^{\mathrm{nc}}(\infty, 1)_{\text {Conn }}^{\bullet}$, then $H(t, x) \in \psi_{\theta}^{\mathrm{nc}}(\infty, 1)_{\text {Conn }}^{\bullet}$ for all $t$. 
Proof For $i \in\{1, \ldots, r\}$, let $[0,1]^{\{i\}} \subseteq[0,1]^{r}$ be the subspace where all coordinates but the $i$-th are 0 . From the paths $p_{i}$, the proof of Proposition 4.16 constructs homotopies $F_{i}:[0,1]^{\{i\}} \times V_{i} \rightarrow \psi_{\theta}(\infty, 1)$ in two steps. First we used the path $p_{i}$ to construct a map

$$
e_{i}:[0,1]^{\{i\}} \times V_{i} \times \Lambda_{i} \times S^{0} \times D^{d} \rightarrow \Gamma\left(f_{V_{i}}\right),
$$

over $V_{i}$ that is an embedding for each point of $[0,1]^{\{i\}} \times V_{i}$, then we let $F_{i}$ be the composition

$$
\begin{aligned}
{[0,1]^{\{i\}} \times V_{i} } & \rightarrow \psi_{\theta}(\infty, 1) \\
(t, x) & \mapsto \mathcal{K}_{w\left(t_{i}\right)}^{e_{i}\left(\tau\left(t_{i}\right), x\right)}(f(x)) .
\end{aligned}
$$

The homotopies $F_{i}$ all start at $s \circ f$ so we can glue them together to a map

$$
\bigcup_{i}\left([0,1]^{\{i\}} \times V_{i}\right) \stackrel{F}{\longrightarrow} \psi_{\theta}(\infty, 1),
$$

where the union is inside $[0,1]^{r} \times X$.

Recall that the embedding $e_{i}(t, x): \Lambda_{i} \times S^{0} \times D^{d} \rightarrow f(x)$ was constructed the following way. On $\{\lambda\} \times\{+1\} \times D^{d}$ it is a disk centered at $p_{i}(x, \lambda, t)$, and on $\{\lambda\} \times\{-1\} \times D^{d}$ it is constructed in the standard strip $L_{\varepsilon} \subseteq f(x)$. By the disjointness assumption (b) we may suppose that the image of $e_{i}(t, x)$ is disjoint from the image of $e_{j}\left(t^{\prime}, x\right)$ for $x \in V_{i} \cap V_{j}$ and $t, t^{\prime} \in[0,1]$, and thus we get an embedding of $\left(\Lambda_{i} \amalg \Lambda_{j}\right) \times S^{0} \times D^{d}$. Disjointness of the $e_{i}$ 's means that we can iterate the construction (4-13). Namely if $T=\left\{i_{1}, \ldots, i_{k}\right\}$ and we set $V_{T}=V_{i_{1}} \cap \cdots \cap V_{i_{k}}$, we have the homotopy

$$
\begin{aligned}
{[0,1]^{T} \times V_{T} } & \rightarrow \psi_{\theta}(\infty, 1) \\
(t, x) & \mapsto \mathcal{K}_{w\left(t_{i_{1}}\right)}^{e_{i_{1}}\left(\tau\left(t_{i_{1}}\right), x\right)} \circ \cdots \circ \mathcal{K}_{w\left(t_{i_{k}}\right)}^{e_{i_{k}}\left(\tau\left(t_{i_{k}}\right), x\right)}(f(x)),
\end{aligned}
$$

where the iterated surgery is defined because the corresponding surgery data are disjoint. Composing (4-15) with the stretching map $s$ gives a homotopy $F_{T}:[0,1]^{T} \times V_{T} \rightarrow$ $\psi_{\theta}(\infty, 1)$. If we regard $[0,1]^{T}$ as a subset of $[0,1]^{r}$ in the obvious way, the various $F_{T}$ 's are compatible and we can glue them to a smooth map extending (4-14)

$$
\bigcup_{T}\left([0,1]^{T} \times V_{T}\right) \stackrel{F}{\rightarrow} \psi_{\theta}(\infty, 1) .
$$

The restriction of $F$ to $\{0\} \times X$ is $s \circ f$, and if $F(t, x)$ is defined and $t_{i}=1$, then $F(t, x)$ is connected at level $a_{i}$. 
Finally we pick for each $i$ a bump function $\rho_{i}: X \rightarrow[0,1]$ supported in $V_{i}$, such that $U_{i} \subseteq \rho_{i}^{-1}(1)$. Let $\rho: X \rightarrow[0,1]^{r}$ have the $\rho_{i}$ as coordinate functions. Then the map

$$
\begin{aligned}
{[0,1] \times X } & \rightarrow[0,1]^{r} \times X \\
(t, x) & \mapsto(t \rho(x), x)
\end{aligned}
$$

has image in the domain of (4-16), and we let $H$ be the composition of the two maps. $\square$

We now return to the proof of Theorem 4.14. Given a map of pairs

$$
\left(D^{k}, \partial D^{k}\right) \stackrel{f}{\rightarrow}\left(\psi_{\theta}^{\mathrm{nc}}(\infty, 1)^{\bullet}, \psi_{\theta}^{\mathrm{nc}}(\infty, 1)_{\text {Conn }}^{\bullet}\right),
$$

we must produce a relative homotopy, deforming $f$ to a map into $\psi_{\theta}^{\mathrm{nc}}(\infty, 1)_{\mathbf{C o n n}}^{\bullet}$. The homotopy will be produced by Corollary 4.17 once we construct the data $\left(V_{i}, a_{i}, p_{i}\right)$. We first consider the case $k=0$, in which the map $p: D^{0} \times \Lambda \times[0,1] \rightarrow W$ is produced by the following lemma. It is proved by first producing paths $p(\lambda,-)$ which are embedded but not necessarily disjoint, and then making them disjoint in Lemma 4.19.

Lemma 4.18 For any $W \in \psi_{\theta}^{\mathrm{nc}}(n, 1)^{\bullet}$ and any $a \in(-1,1)$ which is a regular value of $x_{1}: W \rightarrow \mathbb{R}$, there is a finite set $\Lambda=\left\{\lambda_{1}, \ldots, \lambda_{r}\right\}$ and an embedding $p: \Lambda \times[0,1] \rightarrow W$ such that:

(i) The path $p(\lambda,-):[0,1] \rightarrow W$ starts somewhere in $W \cap x_{1}^{-1}(a)$, outside the path component of the basepoint, and each nonbasepoint component contains exactly one of the starting points $p(\lambda, 0)$.

(ii) The path $p(\lambda,-):[0,1] \rightarrow W$ ends somewhere in $f(x)-x_{1}^{-1}([-10,10])$.

Proof First pick points $p(\lambda, 0) \in W \cap x_{1}^{-1}(a)$, one in each nonbasepoint component. The index set $\Lambda$ is then the set of nonbasepoint components. By assumption, no compact component of $W$ is contained in $x_{1}^{-1}([-10,10])$, so for each $\lambda$ there is a path in $W$ from $p(\lambda, 0)$ to a point in $W-x_{1}^{-1}([-10,10])$. Pick an embedded such path $p(\lambda,-):[0,1] \rightarrow W$. These assemble to a map

$$
p: \Lambda \times[0,1] \rightarrow W,
$$

but it need not be an embedding because the images of the paths $p(\lambda,-)$ need not be disjoint. However, by transversality we may suppose that the paths $p(\lambda,-)$ do not intersect at their endpoints. In Lemma 4.19 below we will prove that we can change each $p(\lambda,-):[0,1] \rightarrow W$ by an isotopy of embedded paths such that the isotoped paths are disjoint. 
Lemma 4.19 Let $W$ be a smooth manifold and $p_{1}, \ldots, p_{r}:[0,1] \rightarrow W$ a set of embedded paths, such that

$$
p_{i}([0,1]) \cap p_{j}(\partial[0,1])=\varnothing \quad \text { for } i \neq j .
$$

Then there are paths $q_{i}:[0,1] \rightarrow W$ such that $p_{i}$ is isotopic to $q_{i}$ through an isotopy of embedded paths which is relative to a neighbourhood of the endpoints, and such that

$$
q_{i}([0,1]) \cap q_{j}([0,1])=\varnothing \quad \text { for } i \neq j .
$$

Proof By induction on $r$ we can assume that $q_{i}=p_{i}$ already have disjoint images for $i<r$. The derivative $q_{i}^{\prime}(t) \in T W$ gives a vector field on $q_{i}([0,1]) \subseteq W$. We can extend this to a vector field $X$ on $W$, with the properties that $X\left(q_{i}(t)\right)=q_{i}^{\prime}(t)$ for $i=1, \ldots, r-1$. Then each $q_{i}$ is the restriction of an integral curve $\bar{q}_{i}$ of $X$. After multiplying $X$ with a function $W \rightarrow[0,1]$ which is 1 on a neighbourhood of the image of the $q_{i}$ 's and vanishes outside a slightly larger neighbourhood, we may assume that $X$ has compact support and that $\bar{q}_{i}$ does not intersect $q_{r}$ outside the image of $q_{i}$.

Finally extend $X$ to a compactly supported vector field on all of $\mathbb{R}^{n}$, ie $X: \mathbb{R}^{n} \rightarrow \mathbb{R}^{n}$. Let $F: \mathbb{R} \times \mathbb{R}^{n} \rightarrow \mathbb{R}^{n}$ be the flow of $X$. It is defined everywhere because $X$ is compactly supported, it preserves $W$, and it also fixes a neighbourhood of the end points of $p_{r}$. Then $q_{r}(s)=F\left(1, p_{r}(s)\right)$ will work, since all intersections between $q_{r}$ and the $\bar{q}_{i}$ 's have been flowed off of $q_{i}$.

Combining Lemma 4.18 and Corollary 4.17 produces a null homotopy of (4-17), proving Theorem 4.14 for $k=0$. For $k>0$ we need a parametrised version of Lemma 4.18.

Proposition 4.20 For any smooth $f: D^{k} \rightarrow \psi_{\theta}^{\mathrm{nc}}(\infty, 1)^{\bullet}$, there exists a covering $D^{k}=V_{1} \cup \cdots \cup V_{k}$ by contractible open sets $V_{i}$, real numbers $a_{i}$, finite sets $\Lambda_{i}$ and embeddings $p_{i}: V_{i} \times \Lambda_{i} \times[0,1] \rightarrow \Gamma\left(\left.f\right|_{V_{i}}\right)$ over $V_{i}$ satisfying the assumptions of Corollary 4.17.

Proof First pick regular values $a_{x} \in(-1,1)$ of $x_{1}: f(x) \rightarrow \mathbb{R}$, one for each $x \in D^{k}$. Apply Lemma 4.18 for $W=f(x)$ and $a=a_{x}$ to find a finite set $\Lambda_{x}$ and an embedding

$$
p_{x}:\{x\} \times \Lambda_{x} \times[0,1] \rightarrow f(x)
$$

satisfying (i) and (ii) of Lemma 4.18.

Then extend to a map $p_{x}: V_{x} \times \Lambda_{x} \times[0,1] \rightarrow \Gamma\left(\left.f\right|_{V_{x}}\right)$ defined on a neighbourhood $V_{x}$ of $x$. After possibly shrinking the $V_{x}$, each tuple $\left(V_{x},\left.f\right|_{V_{x}}, \Lambda_{x}, a_{x}, p_{x}\right)$ will 
satisfy the assumptions in Proposition 4.16. Using compactness of $D^{k}$ we get a finite subcover

$$
D^{k}=V_{1} \cup \cdots \cup V_{r}
$$

with corresponding regular values $a_{i} \in(-1,1)$ and maps

$$
p_{i}: V_{i} \times \Lambda_{i} \times[0,1] \rightarrow \Gamma\left(\left.f\right|_{V_{i}}\right)
$$

satisfying condition (a) of Corollary 4.17 but not the disjointness condition (b).

Before achieving full disjointness of all paths, let us explain how to modify the data $\left(V_{i}, a_{i}, p_{i}\right)$ so as to satisfy

$$
p_{j}\left(\{x\} \times \Lambda_{j} \times \partial[0,1]\right) \text { disjoint from } \operatorname{Im}\left(p_{i}(x,-)\right) \text { for } j<i
$$

To achieve this we proceed by induction on $i$, the case $i=1$ being vacuous. We can temporarily extend $p_{i}$ to a map $e_{i}: V_{i} \times \Lambda_{i} \times[0,1] \times D^{d-1} \rightarrow \Gamma\left(\left.f\right|_{V_{i}}\right)$ over $V_{i}$ that is an embedding of $\{x\} \times \Lambda_{i} \times\{t\} \times D^{d-1}$ into a level set for each $(x, t) \in V_{i} \times[0,1]$. For each $x \in V_{i}$ there is a $d_{x} \in D^{d-1}$ such that $\operatorname{Im}\left(e_{i}\left(x,-, d_{x}\right)\right)$ is disjoint from $\bigcup_{j<i} p_{j}\left(\{x\} \times \Lambda_{j} \times \partial[0,1]\right)$. Furthermore, there is a small contractible neighbourhood $x \in V_{i}^{x} \subseteq V_{i}$ where this is still true. Equip $V_{i}^{x}$ with the paths

$$
p_{i}^{x}=e_{i}\left(-, d_{x}\right): V_{i}^{x} \times \Lambda_{i} \times[0,1] \rightarrow \Gamma\left(\left.f\right|_{V_{i}^{x}}\right)
$$

We can choose finitely many $\left\{x_{j}\right\}_{j \in J}$ such that $\bigcup_{j \in J} V_{i}^{x_{j}}=V_{i}$ and the $d_{x_{j}}$ are distinct points in $D^{d-1}$. Then note that the $\left\{p_{i}^{x_{j}}\right\}_{j \in J}$ have disjoint images in $\Gamma\left(\left.f\right|_{V_{i}}\right)$.

The $\left\{p_{i}^{x_{j}}\right\}_{j \in J}$ all start at height $a_{i} \in(-1,1)$. We wish for the paths to start at different heights: by replacing the paths $p_{i}^{x_{j}}$ with their restrictions to a subinterval $[\eta, 1]$, for some small $\eta>0$, we can ensure that they all start at different heights $a_{i}^{x_{j}}$. We now replace $\left(V_{i}, a_{i}, p_{i}\right)$ by the collection $\left\{\left(V_{i}^{x_{j}}, a_{i}^{x_{j}}, p_{i}^{x_{j}}\right)\right\}_{j \in J}$. This proves the induction step, and hence (4-18).

Finally we pass from the "endpoint disjointness" expressed in (4-18) to the actual disjointness of all paths as required in (b) of Corollary 4.17. This is just an easy parametrised version of Lemma 4.19. Indeed, if we pick an ordering of all the paths involved (ie of the disjoint union of the finite sets $\Lambda_{i}$ ) and proceed by induction as in the proof of that lemma. Suppose we have already made the first $l$ paths disjoint. Then as in the proof of Lemma 4.19 we get in the induction step for each $x \in X$ a compactly supported vector field $X(x): \mathbb{R}^{n} \rightarrow \mathbb{R}^{n}$ preserving $f(x) \subseteq \mathbb{R}^{n}$. It is easy to construct this to depend smoothly on $x$, and then we can flow the $(l+1)$-st path with the flow of $X$ to make it disjoint from the previous paths, without changing any endpoints. 
Proof of Theorem 4.14 Starting with the map of pairs (4-17), Proposition 4.20 produces a collection $\left\{\left(V_{i}, a_{i}, p_{i}\right)\right\}$ satisfying (a) and (b) of Corollary 4.17, which then produces a relative null homotopy.

Proof of Theorem A Lemma 4.6, Lemma 4.7 and Theorem 4.14 imply that the map

$$
\psi_{\theta}^{\mathrm{nc}}(\infty, 1)_{\mathbf{C o n n}}^{\bullet} \rightarrow \psi_{\theta}(\infty, 1)
$$

is a weak homotopy equivalence. Theorem 4.5 and Theorem 3.12 identify these spaces with $B \mathcal{C}_{\theta}^{\bullet}$ and $B \mathcal{C}_{\theta}$ respectively.

\subsection{Reversing morphisms}

Let us study the implications of the assumptions on $\theta: \mathbf{X} \rightarrow B O(2)$. We can arrange that $\mathbf{X}=E O(2) \times O(2) F$, for some space $F$ equipped with an action

$$
O(2) \times F \rightarrow F .
$$

The assumption that $\mathbf{X}$ is path connected says that $\pi_{0} O(2)$ acts transitively on $\pi_{0} F$. Therefore $F$ is either path connected, or has two (homeomorphic) path components which are permuted by the action of some reflection in $O(2)$. To interpret the assumption that $S^{2}$ admits a $\theta$-structure, we consider the induced action of unbased homotopy classes

$$
\left[S^{1}, O(2)\right] \times\left[S^{1}, F\right] \rightarrow\left[S^{1}, F\right] .
$$

We regard $\pi_{0} F \subseteq\left[S^{1}, F\right]$ as the homotopy classes of constant maps.

Proposition 4.21 If $S^{2}$ admits a $\theta$-structure, then the subset $\pi_{0}(F) \subseteq\left[S^{1}, F\right]$ is preserved by elements of $\left[S^{1}, O(2)\right]$ represented by maps of even degree.

That a map $S^{1} \rightarrow O(2)$ has even degree means that the corresponding vector bundle over $S^{2}$ built by clutching has vanishing second Stiefel-Whitney class.

Proof The free homotopy classes of maps of even degree form a subgroup generated by two elements: the element $r$, represented by a constant map $S^{1} \rightarrow O(2)$ to some reflection, and the element $2: S^{1} \rightarrow \mathrm{SO}(2) \subseteq O(2)$ represented by a map of degree 2 . It suffices to see that these preserve $\pi_{0}(F) \subseteq\left[S^{1}, F\right]$. This is obvious for $r$ (as it is represented by a constant map), so we consider 2. Pick a basepoint of $F$ and consider the long exact sequence in homotopy

$$
\pi_{2}(F) \rightarrow \pi_{2} \mathbf{X} \rightarrow \pi_{2} B O(2) \rightarrow \pi_{1} F \rightarrow \pi_{1} \mathbf{X} \rightarrow \cdots .
$$


The assumption says that a classifying map for $T S^{2}$ gives an element of $\pi_{2} B O(2)=$ $\pi_{1} O(2)$ which lifts to $\pi_{2} \mathbf{X}$. Equivalently it must vanish in $\pi_{1} F$. But $T S^{2}$ comes from a map $S^{1} \rightarrow \mathrm{SO}(2) \subseteq O(2)$ which has degree 2 , hence this assumption says that $2 \in\left[S^{1}, O(2)\right]$ acts trivially on $\left[S^{1}, F\right]$.

We next study $\theta$-structures on $D^{2}$. More precisely, if we are given a structure over $\partial D^{2}$, how do we know whether it extends to all of $D^{2}$ ? Such a structure is called a bounding structure, and we prove that the set of bounding structures is preserved by a certain construction that we now define. Let

$$
V \in \Gamma\left(\left.T D^{2}\right|_{\partial D^{2}}\right)
$$

be a nonzero vector field, and let

$$
\phi^{V}:\left.\left.T D^{2}\right|_{\partial D^{2}} \rightarrow T D^{2}\right|_{\partial D^{2}}
$$

be the bundle map which maps $V \mapsto-V$, and acts as the identity on $V^{\perp}$.

\section{Corollary 4.22 Let}

$$
l:\left.T D^{2}\right|_{\partial D^{2}} \rightarrow \theta^{*} \gamma
$$

be a bounding $\theta$-structure (ie one that extends to $D^{2}$ ). Then $l \circ \phi^{V}$ is also bounding.

Proof Give $D^{2}$ the framing coming from $\mathbb{R}^{2}$. With respect to this framing, $l: \partial D^{2} \rightarrow F$ has homotopy class

$$
l \in\left[S^{1}, F\right]
$$

which is in $\pi_{0} F \subseteq\left[S^{1}, F\right]$ because $l$ bounds. With respect to the framing, the bundle map $\phi^{V}$ corresponds to the map $S^{1} \rightarrow O(2)$ which takes $x \in S^{1}$ to the reflection taking $V(x) \mapsto-V(x)$ and preserves $V(x)^{\perp}$. This map depends not on the vector $V(x)$, but only on the line $\mathbb{R} V(x)$, so it factors through the projection $S^{1} \rightarrow \mathbb{R} P^{1}$. It follows that $\phi^{V}$ corresponds to an element

$$
\phi^{V} \in\left[S^{1}, O(2)\right]
$$

which is of even degree. Consequently $l \circ \phi^{V} \in\left[S^{1}, F\right]$ also bounds, by the previous proposition.

We now study the extent to which morphisms in $C_{\theta}$ can be "turned around". The following proposition depends crucially on the assumption that $S^{2}$ admit a $\theta$-structure. It is false for example for framed manifolds. 
Proposition 4.23 Let $c_{0}=\left(M_{0}, l_{0}\right) \in C_{\theta}$ and $c_{1}=\left(M_{1}, l_{1}\right)$ be objects and let $(W, l): c_{0} \rightarrow c_{1}$ be a morphisms in $C_{\theta}$ whose underlying manifold is connected. Then there exists a morphism $(\bar{W}, \bar{l}): c_{1} \rightarrow c_{0}$ whose underlying manifold $\bar{W}$ is diffeomorphic to $W$.

In particular, if $c_{0}$ and $c_{1}$ are linked by a zig-zag of morphisms, then there are in fact morphisms between them in both directions.

Proof Recall that $W \subseteq[0, t] \times \mathbb{R}^{n}$ for some large $n$, and that $W$ is "cylindrical" near its boundary. Let $\varphi:[0, t] \rightarrow[0, t]$ be the affine map $s \mapsto t-s$, and let $F=$ $\varphi \times \operatorname{Id}:[0, t] \times \mathbb{R}^{n} \times[0, t] \times \mathbb{R}^{n}$.

To construct the morphism $c_{1} \rightarrow c_{0}$ we first construct the underlying manifold as $\bar{W}=F^{-1}(W)$. If there were no tangential structures to worry about, this would be a morphism from $c_{1}$ to $c_{0}$, but we need to extend the structure given near $\partial \bar{W}=c_{1} \amalg c_{0}$ to all of $\bar{W}$.

The vector $V=\partial / \partial x_{1} \in \mathbb{R}^{n+1}$ gives a section of $T W$, defined near $\partial W$ (pointing inwards on the incoming boundary and outwards on the outgoing). Since $W$ is connected, we can pick a relative $\mathrm{CW}$ structure with only one 2-dimensional cell. Let $W^{1} \subseteq W$ be the 1 -skeleton in this $\mathrm{CW}$ structure. We can extend $V$ to a nonzero section

$$
V \in \Gamma\left(\left.T W\right|_{W^{1}}\right) .
$$

Now let $\phi^{V}:\left.\left.T W\right|_{W^{1}} \rightarrow T W\right|_{W^{1}}$ be the automorphism constructed as before: it takes $V \mapsto-V$ and is the identity on $V^{\perp}$. The "reflected" tangential structure

$$
\bar{l}=l \circ \phi^{V}:\left.T W\right|_{W^{1}} \rightarrow \theta^{*} \gamma,
$$

extends first to a neighbourhood of $W^{1} \subseteq W$, and then over the 2-cell $D^{2} \rightarrow W$ by the corollary above.

Thus we have a tangential structure $\bar{l}: T W \rightarrow \theta^{*} \gamma$. If we use the diffeomorphism $F: W \rightarrow \bar{W}$ to transport it to a structure on $\bar{W}$, we have produced the required morphisms $c_{1} \rightarrow c_{0}$.

\subsection{Proof of Theorem B}

By Theorem 4.5, we have reduced the first part of Theorem B to the following theorem.

Theorem 4.24 For tangential structures $\theta: \mathbf{X} \rightarrow B O(2)$ such that $\mathbf{X}$ is path connected and $S^{2}$ admits a $\theta$-structure, the inclusion

$$
\psi_{\theta}^{\mathrm{nc}}(\infty, 1)_{\mathbf{C}}^{\bullet} \rightarrow \psi_{\theta}^{\mathrm{nc}}(\infty, 1)_{\mathbf{C} \text { onn }}^{\bullet}
$$

is a weak homotopy equivalence of each component onto a component of $\psi_{\theta}^{\mathrm{nc}}(\infty, 1)_{\text {Conn }}^{\bullet}$. 
The second part of Theorem B will be proved in Proposition 4.27. Let us first prove Theorem 4.24 in the case where $\mathbf{C}$ is a disjoint union of path components of $\operatorname{ob}\left(C_{\theta}^{\bullet}\right)$. We will explain how to remove this assumption in Proposition 4.26.

Suppose we are given a map of pairs

$$
f:\left(D^{k}, \partial D^{k}\right) \rightarrow\left(\psi_{\theta}^{\mathrm{nc}}(\infty, 1)_{\mathbf{C o n n}}^{\bullet}, \psi_{\theta}^{\mathrm{nc}}(\infty, 1)_{\mathbf{C}}^{\bullet}\right) .
$$

For each point $x \in D^{k}$, we can choose a regular value $a_{x} \in(-1,1)$ of $x_{1}: f(x) \rightarrow \mathbb{R}$. There is an open neighbourhood $U_{x}$ of $x$ on which $a_{x}$ is still regular. We may choose a finite subcover $\left\{U_{i}\right\}_{i \in I}$ of $\left\{U_{x}\right\}_{x \in X}$, and suppose that the $a_{i}$ are distinct: otherwise we may perturb them so that they are. Choose an $\varepsilon>0$ such that the intervals $\left(a_{i}-2 \varepsilon, a_{i}+2 \varepsilon\right)$ are disjoint. By Lemma 3.4, we may further suppose that for $x \in U_{i}$ the manifold $f(x)$ is cylindrical in $x_{1}^{-1}\left(a_{i}-\varepsilon, a_{i}+\varepsilon\right)$.

As we are working in the space of manifolds with basepoint, and the family $D^{k}$ is compact, there is a $\delta>0$ such that the strip $L_{3 \delta}=\mathbb{R} \times(-3 \delta, 3 \delta) \times\{0\}$ lies inside every manifold $f(x)$, and furthermore that $f(x) \cap \mathbb{R} \times(-3 \delta, 3 \delta)^{\infty}=L_{3 \delta}$. Recall that inside $L_{3 \delta}$ the surface has the standard $\theta$-structure. We can change the manifold inside $\mathbb{R} \times(\delta, 2 \delta) \times(-3 \delta, 3 \delta)^{\infty-1}$ without leaving the space of manifolds with basepoint, as the new manifold will still contain $L_{\delta}$.

The set $\pi_{0} \mathrm{ob}\left(\mathcal{C}_{\theta}^{\bullet}\right)$ is in natural bijection with $\pi_{1} \operatorname{Fib}(\theta)$, as an object $C$ has a natural framing of $\varepsilon^{1} \oplus T C$, extending the given framing on $(-\varepsilon, \varepsilon) \times\{0\}$, and this framing is unique up to homotopy. Write $*$ for the object having the standard $\theta$-structure with respect to this framing. For each $U_{i}$, choosing a $u_{i} \in U_{i}$ and setting $M_{i}=$ $f\left(u_{i}\right) \cap\left\{a_{i}\right\} \times \mathbb{R}^{\infty}$ produces a homotopy class $\left[M_{i}\right] \in \pi_{1} \operatorname{Fib}(\theta)$ which is independent of the choice of $u_{i}$.

Lemma 4.25 Let $\mathbf{C} \subseteq \mathrm{ob}\left(\mathcal{C}_{\theta}^{\bullet}\right)$ be a disjoint union of path components: this determines a subset $\pi_{0} \mathbf{C} \subseteq \pi_{1} \operatorname{Fib}(\theta)$. If an object $M \in \mathcal{C}_{\theta}^{\bullet}$ lies in a component of $B \mathcal{C}_{\theta}^{\bullet}$ that also contains an object in $\mathbf{C}$, there is another object $N$ such that $[M] \cdot[N] \in \pi_{0} \mathbf{C} \subseteq \pi_{1} \operatorname{Fib}(\theta)$, where $\cdot$ denotes the group product in $\pi_{1} \operatorname{Fib}(\theta)$. Furthermore, there are morphisms

$$
* \rightarrow N \rightarrow *
$$

in $\mathcal{C}_{\theta}^{\bullet}$. If $M$ is already in $\mathbf{C}$, we can choose $N$ to be $*$.

Proof Choose morphisms $E_{1}: M \rightarrow C \in \mathbf{C}$, and $E_{2}: C \rightarrow M$ which exist by Proposition 4.23, and an object $M^{-1}$ such that $\left[M^{-1}\right]=[M]^{-1} \in \pi_{1} \operatorname{Fib}(\theta)$. Scale the morphisms $E_{1}$ and $E_{2}$ to have length 1 . Consider the morphism $I_{M^{-1}}=$ $\left(1, \mathbb{R} \times M^{-1}\right): M^{-1} \rightarrow M^{-1}$. We may cut this along $\mathbb{R} \times\{0\}$ to obtain a surface with 
$\theta$-structure and height function, $\widetilde{I}_{M^{-1}}$, having two identical boundary components, which we will call $\widetilde{M^{-1}}$ and which are intervals with a $\theta_{1}$-structure that is standard near their ends.

Let $\varepsilon>0$ be small enough such that both of the morphisms $E_{1}$ and $E_{2}$ satisfy $E_{i} \cap \mathbb{R} \times(-3 \varepsilon, 3 \varepsilon)^{\infty}=L_{3 \varepsilon}$. In particular, the morphisms come from $\mathcal{C}_{\theta}^{3 \varepsilon}$. There is a height-preserving embedding $e: \widetilde{I}_{M^{-1}} \rightarrow \mathbb{R} \times[\varepsilon, 2 \varepsilon] \times[0,3 \varepsilon)^{\infty}$ sending $\partial \tilde{I}_{M^{-1}}$ to $\mathbb{R} \times\{\varepsilon, 2 \varepsilon\} \times\{0\}$ with image that agrees with $L_{3 \varepsilon}$ as a $\theta$-manifold near $(\mathbb{R} \times \mathbb{R}-$ $(\varepsilon, 2 \varepsilon)) \times \mathbb{R}^{\infty}$

Let

$$
\widetilde{E}_{i}=\left(E_{i}-\mathbb{R} \times(\varepsilon, 2 \varepsilon) \times\{0\}\right) \cup e\left(\widetilde{I}_{M^{-1}}\right) \in \psi_{\theta}^{\mathrm{nc}}(\infty, 1)_{\text {Conn }}^{\bullet} .
$$

Then $\left(1, \widetilde{E}_{1}\right)$ and $\left(1, \widetilde{E}_{2}\right)$ are morphisms in $\mathcal{C}_{\theta}^{\bullet}$ between $(M-(\varepsilon, 2 \varepsilon) \times\{0\}) \cup e\left(\widetilde{M^{-1}}\right)$ and $(C-(\varepsilon, 2 \varepsilon) \times\{0\}) \cup e\left(\widetilde{M^{-1}}\right)$. The first of these objects is in the path component of $*$, and the second is defined to be $N$.

We will now construct a $\theta$-manifold $D$ with height function, which we will glue into the manifolds $f(x)$ inside $L_{3 \delta}$, similarly to the proof of Lemma 4.25. Let $C_{i}$ be the composition $* \rightarrow N_{i} \rightarrow *$ obtained from the above Lemma applied to $M_{i}$, and scaled so that each morphism has length $\varepsilon$. Let $\widetilde{C}_{i}$ denote the $\theta$-manifold with height function obtained by cutting $C_{i}$ along $\mathbb{R} \times\{0\}$, and adding $a_{i}-\varepsilon$ to the height function. The boundary of $\widetilde{C}_{i}$ can be naturally identified with the boundary of $\mathbb{R} \times[0,1]$, where the height function is given by the first coordinate. There is a height-preserving embedding $e_{i}: \widetilde{C}_{i} \rightarrow \mathbb{R} \times[\delta, 2 \delta] \times[0,3 \delta)^{\infty}$ sending $\partial \widetilde{C}_{i}$ to $\mathbb{R} \times\{\delta, 2 \delta\}$ with image that agrees with $L_{3 \delta}$ as a $\theta$-manifold near the boundary of $\left[a_{i}-\varepsilon, a_{i}+\varepsilon\right] \times[\delta, 2 \delta] \times \mathbb{R}^{\infty}$.

Let $D$ be the $\theta$-manifold obtained from $L_{3 \delta}$ by cutting out $\left[a_{i}-\varepsilon, a_{i}+\varepsilon\right] \times[\delta, 2 \delta] \times\{0\}$ and gluing in $e_{i}\left(\widetilde{C}_{i}\right) \cap\left[a_{i}-\varepsilon, a_{i}+\varepsilon\right] \times[\delta, 2 \delta] \times \mathbb{R}^{\infty}$. Figure 5 shows the part of $D$ near $\mathbb{R} \times(\delta, 2 \delta) \times \mathbb{R}^{\infty}$.

For each $x \in D^{k}$ we can form $\bar{f}(x)=\left(f(x)-L_{3 \delta}\right) \cup D$, which defines a continuous map $\bar{f}: D^{k} \rightarrow \psi_{\theta}(\infty, 1)_{\text {Conn }}^{\bullet}$. If $x \in U_{i}$, then $\left[\bar{f}(x) \cap\left\{a_{i}\right\} \times \mathbb{R}^{\infty}\right]=\left[M_{i}\right] \cdot\left[N_{i}\right] \in$ $\pi_{1} \operatorname{Fib}(\theta)$ which is in $\pi_{0} \mathbf{C}$ by construction. Thus $\bar{f}$ is a map into $\psi_{\theta}(\infty, 1)_{\mathbf{C}}^{\bullet}$, and we must show it is relatively homotopic to $f$. We will do so by changing the height function on $D$. For ease of notation we will write $h$ instead of $x_{1}$ for the height function.

We first deform the height function on $D$ so that $D-L_{3 \delta}$ has total height 0 . Let $c_{1}: \mathbb{R} \rightarrow \mathbb{R}$ be a smooth function such that $\left(t_{i}-\varepsilon, t_{i}+\varepsilon\right) \subseteq c_{1}^{-1}\left(t_{i}\right)$, and $c_{1}^{\prime} \geq 0$. Let $c_{s}=s \cdot c_{1}+(1-s) \cdot$ Id. Choose a smooth function $\varphi$ on $D$ that is identically 1 on 


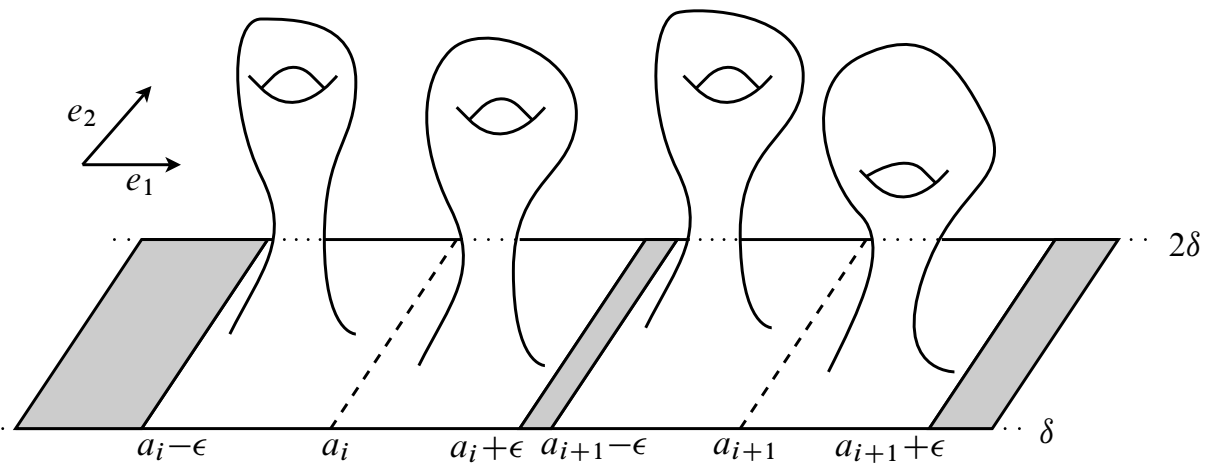

Figure 5

$D-L_{3 \delta}$, and identically 0 near $\mathbb{R} \times \mathbb{R}-(\delta, 2 \delta) \times \mathbb{R}^{\infty}$. We can modify the height function on $D$ by

$$
h_{s}(d)=\varphi(d) \cdot\left(c_{s} \circ h(d)-h(d)\right)+h(d) .
$$

Then $h_{0}=h$ and if $c \in e_{i}\left(\widetilde{C}_{i}\right)-L_{3 \delta} \subseteq D$ then $h_{1}(c)=t_{i}$. The level sets of $h_{1}$ in $D$ at any points except $\left\{t_{1}, \ldots, t_{n}\right\}$ are then intervals with the standard $\theta$-structure.

We now define another path of height functions on $D$,

$$
h_{s}^{\prime}(d)=\varphi(d) \cdot 20 \cdot s+h_{1}(d) .
$$

Then $h_{0}^{\prime}=h_{1}$. If $c \in e_{i}\left(\widetilde{C}_{i}\right)-L_{3 \delta} \subseteq D$ then $h_{1}^{\prime}(d)=t_{i}+20 \in(19,21)$. With this height function, $D$ has the standard tangential structure between heights -10 and 10 .

Applying these paths of height functions to the copy of $D$ inside each $\bar{f}(x)$ gives a path from $\bar{f}(x)$ to a manifold that agrees with $f(x)$ inside $x_{1}^{-1}(-10,10)$, so composing with the stretching map (4-11) gives a path to $f(x)$.

We must explain why these are relative homotopies. Suppose $f(x) \in \psi_{\theta}^{\mathrm{nc}}(\infty, 1)_{\mathbf{C}}^{\bullet}$, so there is a $t \in(-1,1)$ and an $\eta>0$ with $f(x) \cap\left\{t^{\prime}\right\} \times \mathbb{R}^{\infty} \in \mathbf{C}$ for all $t^{\prime} \in(t-\eta, t+\eta)$. If $t$ is not in $\bigcup_{i}\left(a_{i}-\varepsilon, a_{i}+\varepsilon\right)$, then $\bar{f}(x)$ agrees with $f(x)$ near $t$, so the level set at $t$ of $\bar{f}(x)$ is in $\mathbf{C}$. The path $h_{s}$ only changes the level sets in $\bigcup_{i}\left(a_{i}-\varepsilon, a_{i}+\varepsilon\right)$, so also always has a level set in $\mathbf{C}$. Finally, the path $h_{s}^{\prime}$ only has finitely many level sets different from those of $f(x)$ at any time. Thus the final manifold always has a $t^{\prime} \in(t-\eta, t+\eta)$ with level set in $\mathbf{C}$.

On the other hand, suppose $t \in\left(a_{i}-\varepsilon, a_{i}+\varepsilon\right)$. Then $M_{i} \in \mathbf{C}$ already, and we have thus chosen $N_{i}$ to be trivial. Thus the level sets of $f(x)$ and $\bar{f}(x)$ at $t$ are the same, and in $\mathbf{C}$. The path $h_{s}$ does not change this. Finally, the path $h_{s}^{\prime}$ only has finitely many 
level sets in $(-10,10)$ different from $f(x)$ at a time. Thus the final manifold always has a $t^{\prime}$ with level set in $\mathbf{C}$.

This concludes the proof of Theorem 4.24, in the case that $\mathbf{C}$ consists of whole path components of $\psi_{\theta_{d-1}}(\infty, 0)$. The general case follows by:

Proposition 4.26 Let $\mathbf{C}$ be a collection of elements of $\psi_{\theta_{d-1}}(\infty, 0)$, and $\mathbf{C}^{\prime}$ be the collection of all elements of $\psi_{\theta_{d-1}}(\infty, 0)$ in the same path component as an element in $\mathbf{C}$. Then the inclusion

$$
\psi_{\theta}^{\mathrm{nc}}(\infty, 1)_{\mathbf{C}}^{\bullet} \rightarrow \psi_{\theta}^{\mathrm{nc}}(\infty, 1)_{\mathbf{C}^{\prime}}^{\bullet}
$$

is a weak homotopy equivalence.

Proof We will show that the relative homotopy groups of the inclusion vanish. The argument is almost identical to that of Proposition 3.21, so we will explain to what extent it differs.

Take a map $f:\left(D^{m}, \partial D^{m}\right) \rightarrow\left(\psi_{\theta}^{\mathrm{nc}}(\infty, 1)_{\mathbf{C}^{\prime}}^{\bullet}, \psi_{\theta}^{\mathrm{nc}}(\infty, 1)_{\mathbf{C}}^{\bullet}\right)$. As usual, there is a finite cover of $D^{m}$ by contractible open sets $U_{i}$ and values $a_{i} \in(-1,1)$ such that $a_{i}$ is a regular value of the height function on $f(x)$ for $x \in U_{i}$, and the level sets at $a_{i}$ are in $\mathbf{C}^{\prime}$. We may suppose the $a_{i}$ are distinct, and choose an $\varepsilon>0$ so that the intervals $\left(a_{i}-2 \varepsilon, a_{i}+2 \varepsilon\right)$ are disjoint. By Lemma 3.4, we may further suppose that $f\left(x \in U_{i}\right)$ is cylindrical in $x_{1}^{-1}\left(a_{i}-\varepsilon, a_{i}+\varepsilon\right)$. It is important to note that the homotopy of Lemma 3.4 does not change which level sets occur in $f(x)$, only the heights at which they occur: thus it gives a relative homotopy.

Thus there is a map $\lambda_{i}: U_{i} \rightarrow \psi_{\theta_{d-1}}(\infty, 0)^{\bullet}$ so that $f(x)$ and $\mathbb{R} \times \lambda_{i}(x)$ are equal in $\Psi_{\theta}\left(x_{1}^{-1}\left(a_{i}-\varepsilon, a_{i}+\varepsilon\right)\right)$. The map $\lambda_{i}$ takes values in $\mathbf{C}^{\prime}$. As $U_{i}$ is contractible we can pick a smooth homotopy $\Lambda_{i}:[0,1] \times U_{i} \rightarrow \mathbf{C}^{\prime}$ with $\Lambda_{i}(0,-)=\lambda_{i}$ and $\Lambda_{i}(1,-) \in \mathbf{C}$. We use these functions exactly as in the proof of Proposition 3.21.

It remains to see why this gives a relative homotopy. Over $U_{i}$, elements $f(x)$ are cylindrical in $x_{1}^{-1}\left(a_{i}-\varepsilon, a_{i}+\varepsilon\right)$. The homotopy constructed in Proposition 3.21 is constant outside of $x_{1}^{-1}\left(a_{i}-2 \delta, a_{i}+2 \delta\right)$, for some $0<3 \delta<\varepsilon$. In particular, throughout the homotopy there are only new types of level sets added: if $f(x)$ has a level set in $\mathbf{C}$, then either it occurs at a height outside of $\left(a_{i}-2 \delta, a_{i}+2 \delta\right)$ and so is unchanged by the homotopy, or it occurs at a height inside $\left(a_{i}-2 \delta, a_{i}+2 \delta\right)$, in which case it also occurs at a height inside $\left(a_{i}+2 \delta, a_{i}+\varepsilon\right)$, which must be unchanged by the homotopy.

Theorem 4.24 implies the first part of Theorem B. It remains to see homotopy commutativity of the endomorphism monoids in $\mathcal{C}_{\theta}^{\bullet}$; to that end we give yet another model for these spaces. 
The monoid of endomorphisms of an object $C \in \mathcal{C}_{\theta}^{\bullet}$ is homotopy equivalent to $\bigsqcup_{W} B \operatorname{Diff}_{\theta}(W, L \cup \partial W)$, where $W$ ranges over connected compact $\theta$-surfaces with two boundary circles, both identified with $C$. We can cut such surfaces along $L$ to obtain surfaces with one boundary component. More precisely, given a pointed map $l: S^{1} \rightarrow \operatorname{Fib}(\theta)$, define

$$
\tilde{l}: \mathbb{R}^{2} \stackrel{\pi_{2}}{\rightarrow} \mathbb{R} \rightarrow S^{1} \stackrel{l}{\rightarrow} \operatorname{Fib}(\theta)
$$

where $\mathbb{R} \rightarrow S^{1}$ collapses the complement of $(0,1)$ to the basepoint. Define an element $B_{l}=\left(\mathbb{R}^{2} \times\{0\}, \widetilde{l}\right) \in \Psi_{\theta}\left(\mathbb{R}^{\infty}\right)$. Let $\mathcal{M}(l) \subseteq \Psi_{\theta}\left(\mathbb{R}^{\infty}\right) \times \mathbb{R}$ be the subspace of pairs $(W, t)$ where $W$ is connected and agrees with $B_{l}$ on an open neighbourhood of the complement of $(0, t) \times(0,1) \times \mathbb{R}^{\infty}$. This is a monoid via

$$
\left(W_{1}, t_{1}\right) \circ\left(W_{2}, t_{2}\right)=\left(W_{1} \cup\left(W_{2}+t_{1} \cdot e_{1}\right), t_{1}+t_{2}\right) .
$$

An object $C \in \mathcal{C}_{\theta}^{\bullet}$ determines a loop $l_{C}: S^{1} \rightarrow \operatorname{Fib}(\theta)$, after choosing a parametrisation of the underlying circle (of which there are a contractible choice, as it is determined near the basepoint). There is an object $C^{\prime}$ in the same path component of ob $\left(\mathcal{C}_{\theta}^{\bullet}\right)$ as $C$ and a map of monoids $\mathcal{M}\left(l_{C}\right) \rightarrow \operatorname{End}_{\mathcal{C}_{\theta}^{\bullet}}\left(C^{\prime}\right)$ given by replacing $B_{l}$ by a cylinder $\mathbb{R} \times C^{\prime}$ that agrees with $B_{l}$ near $\mathbb{R} \times[0,1] \times\{0\}$. It is a weak homotopy equivalence as they are classifying spaces for equivalent families. Furthermore, the monoids $\operatorname{End}_{\mathcal{C}_{\theta}^{\bullet}}\left(C^{\prime}\right)$ and $\operatorname{End}_{\mathcal{C}_{\theta}^{\bullet}}(C)$ are equivalent in the following sense: choosing a path $C \rightsquigarrow C^{\prime}$ in ob $\left(\mathcal{C}_{\theta}^{\bullet}\right)$ gives a weak homotopy equivalence $\operatorname{End}_{\mathcal{C}_{\theta}^{\bullet}}\left(C^{\prime}\right) \rightarrow \operatorname{End}_{\mathcal{C}_{\theta}^{\bullet}}(C)$ that is not quite a map of monoids, but is a map of $H$-spaces (and in fact a map of $A_{\infty}$ spaces).

Proposition 4.27 Let $\theta: \mathbf{X} \rightarrow B O(2)$ be any tangential structure. Then the monoid of endomorphisms of an object $C \in \mathcal{C}_{\theta}^{\bullet}$ is homotopy commutative.

Proof By the above discussion, it is enough to prove the homotopy commutativity of $\mathcal{M}(l)$. Consider first the case where $l$ is the constant map to $* \in \operatorname{Fib}(\theta)$, the basepoint which determines the standard tangential structure. Then $\tilde{l}=*$ also and so $B_{l}=\mathbb{R}^{2} \times\{0\}$ with the standard $\theta$-structure induced by the framing given by the coordinate directions. Thus $\mathcal{M}(*)$ has an obvious action of the little 2 -cubes operad. Its usual monoid multiplication is homotopic to that induced by the operad, so its usual multiplication is homotopy commutative (in fact, $E_{2}$ ).

There is a map $l_{*}: \mathcal{M}\left(l_{1}\right) \rightarrow \mathcal{M}\left(l \cdot l_{1}\right)$ given on $(W, t)$ by scaling $W-B_{l_{1}}$ by $\frac{1}{2}$ in the $e_{2}$ direction, and then taking the union with $B_{l \cdot l_{1}}$. This is a map of monoids, and composing with $l_{*}^{-1}$ gives a map $\mathcal{M}\left(l_{1}\right) \rightarrow \mathcal{M}\left(l^{-1} \cdot l \cdot l_{1}\right)$. This is easily seen to have a homotopy inverse, by picking a null homotopy of $l^{-1} \cdot l$ (though the inverse is not a map of monoids). Thus the maps $l_{*}$ are homotopy equivalences. In particular $\mathcal{M}(l) \rightarrow$ $\mathcal{M}\left(l^{-1} \cdot l\right) \simeq \mathcal{M}(*)$ is a monoid map and an equivalence, so $\mathcal{M}(l)$ is equivalent as a monoid to a homotopy commutative monoid, so is homotopy commutative itself. 


\section{Applications}

As mentioned in the introduction, taking the tangential structure $\theta: B \mathrm{SO}(2) \rightarrow B O(2)$ gives a category $\mathcal{C}_{\mathrm{SO}(2)}^{\bullet}$ having a unique path component, and hence cobordism class, of objects. The endomorphism monoid of any object is homotopy equivalent to $\mathcal{M}=$ $\bigsqcup_{g \geq 0} B \operatorname{Diff}^{+}\left(F_{g}, \partial F_{g}=S^{1}\right)$ with the pair of pants product. By [3], components of the group $\operatorname{Diff}^{+}\left(F_{g}, \partial F_{g}=S^{1}\right)$ are contractible, so we can replace it by the associated mapping class group $\Gamma_{g, 1}=\pi_{0} \operatorname{Diff}^{+}\left(F_{g}, \partial F_{g}=S^{1}\right)$. The discussion in Section 1.1 proves:

Corollary C (Madsen-Weiss [10]) There is a homology equivalence

$$
\mathbb{Z} \times B \Gamma_{\infty, 1} \rightarrow \Omega^{\infty} \operatorname{MTSO}(2),
$$

where $\Gamma_{\infty, 1}$ is the limit of the mapping class groups $\Gamma_{g, 1}$ as $g \rightarrow \infty$.

A similar result holds for nonorientable or spin surfaces, though the components of the relevant monoid are slightly more complicated.

Corollary D (Wahl [15, Theorem B]) Let $\mathcal{N}_{g}$ be the mapping class group of a nonorientable surface of genus $g$. Then there is a homology equivalence

$$
\mathbb{Z} \times B \mathcal{N}_{\infty} \rightarrow \Omega^{\infty} \mathbf{M T O}(2) .
$$

Proof We apply Theorem B with no tangential structure. The category $\mathcal{C}_{O(2)}^{\bullet}$ has a unique cobordism class of objects. The relevant homotopy commutative monoid is

$$
\mathcal{M}=\coprod_{F} B \operatorname{Diff}\left(F, \partial F=S^{1}\right)
$$

where the disjoint union is over all diffeomorphism types of unoriented surfaces with one boundary component, and Theorem B identifies its classifying space as $\Omega^{\infty-1} \mathbf{M T O}(2)$. The monoid $\pi_{0} \mathcal{M}$ is in bijection with the set of pairs $\left(g_{1}, g_{2}\right) \in \mathbb{N}^{2}$ where one entry is always 0 . The element $(g, 0)$ corresponds to $F_{g, 1}=\#^{g} T-D^{2}$ and $(0, g)$ corresponds to $N_{g, 1}=\#^{g} \mathbb{R} \mathbb{P}^{2}-D^{2}$. Then addition in this monoid is given by usual addition if both elements are in the same factor, and by the formula $(g, 0)+(0, h>0)=(0, h+2 g)$ otherwise. This may be group completed by inverting the element $(0,1)$. So the homology of the group completion of $\mathcal{M}$ is the same as that of the telescope $\mathcal{M}_{\infty}$ of

$$
\mathcal{M} \stackrel{\cdot(0,1)}{\longrightarrow} \mathcal{M} \stackrel{\cdot(0,1)}{\longrightarrow} \mathcal{M} \stackrel{\cdot(0,1)}{\longrightarrow} \cdots .
$$

There is a submonoid $\mathcal{M}^{\prime}$ of $\mathcal{M}$ consisting only of the nonorientable surfaces (and the disc), and translation by $(0,1)$ sends $\mathcal{M}$ into $\mathcal{M}^{\prime}$, so $\mathcal{M}^{\prime}$ is cofinal. Thus $\mathcal{M}_{\infty} \simeq \mathcal{M}_{\infty}^{\prime}$ 
and this is equivalent to $\mathbb{Z} \times B \mathcal{N}_{\infty}$, the classifying space of the stable nonorientable mapping class group.

Corollary E (Harer [8], Bauer [1]) Let $\mathfrak{s}$ be a spin structure on $F_{g, 1}$ which agrees with the trivial spin structure on the boundary circle, and let $\Gamma_{g, 1}^{\mathfrak{s}}$ be the spin mapping class group, ie the subgroup of $\Gamma_{g, 1}$ which preserves $\mathfrak{s}$.

There is a homology equivalence

$$
\mathbb{Z} \times \mathbb{Z} / 2 \times B \Gamma_{\infty}^{\sigma} \rightarrow \Omega^{\infty} \operatorname{MTSpin}(2)
$$

where $\Gamma_{\infty, 1}^{\sigma}$ is the stable spin mapping class group.

Proof We apply Theorem B with the tangential structure $\theta: B \operatorname{Spin}(2) \rightarrow B O(2)$. Write $\gamma^{s}$ for the universal vector bundle over $B \operatorname{Spin}(2)$. Note that $B \operatorname{Spin}(2) \simeq B \operatorname{SO}(2)$ is connected and $S^{2}$ admits a spin structure, so the hypotheses of the theorem are fulfilled. The category $\mathcal{C}_{\text {Spin(2) }}^{\bullet}$ has two cobordism classes of objects, corresponding to a trivial and a nontrivial spin structure on the circle. The monoid of endomorphisms of the trivial circle is

$$
\mathcal{M}=\coprod_{F} B \operatorname{Diff}^{\operatorname{Spin}}(F, \partial F)=\coprod_{F} \operatorname{Bun}^{\partial}\left(T F, \gamma^{s}\right) / / \operatorname{Diff}(F, \partial F),
$$

where the disjoint union is over all diffeomorphism types of surfaces with one boundary component. If $F$ is not orientable, $\operatorname{Bun}^{\partial}\left(T F, \gamma^{s}\right)=\varnothing$ and it plays no role. If $F$ is an oriented surface, the space $\operatorname{Bun}^{\partial}\left(T F, \gamma^{s}\right)$ is homotopy equivalent to the space of pairs $(\tau, l)$ of a map $\tau: T F \rightarrow \gamma^{+}$classifying the oriented tangent bundle and a relative lift $l$ of the underlying map $\bar{\tau}: F \rightarrow B S O(2)$,

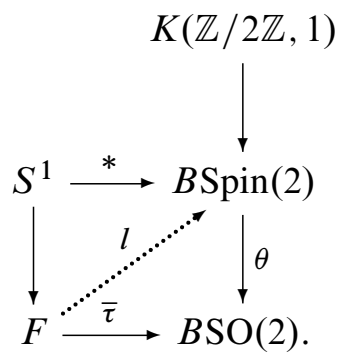

The space of classifying maps $\tau$ is contractible, and for a fixed $\tau$ the space of lifts $l$ is a torsor for the topological abelian group $\operatorname{Map}((F, \partial F),(K(\mathbb{Z} / 2 \mathbb{Z}, 1), *))$, which has contractible path components. It follows that $\operatorname{Bun}^{\partial}\left(T F, \gamma^{s}\right)$ has contractible path components, and we write $\operatorname{Spin}(F, \partial F)=\pi_{0}\left(\operatorname{Bun}^{\partial}\left(T F, \gamma^{s}\right)\right)$. Thus

$$
B \operatorname{Diff}^{\operatorname{Spin}}(F, \partial F) \simeq \operatorname{Spin}(F, \partial F) / / \operatorname{Diff}^{+}(F, \partial F) .
$$


The group $\operatorname{Diff}^{+}(F, \partial F)$ acts on $\operatorname{Spin}(F, \partial F)$ with two orbits (distinguished by their Arf invariant). Pick representatives $\mathfrak{s}_{0}$ and $\mathfrak{s}_{1}$ for these orbits. If $\operatorname{Diff}{ }^{\mathfrak{s}_{i}}(F, \partial F)$ is the stabiliser of $\mathfrak{s}_{i} \in \operatorname{Spin}(F, \partial F)$, then there is an equivalence

$$
B \operatorname{Diff}^{\operatorname{Spin}}(F, \partial F) \simeq B \operatorname{Diff}^{\mathfrak{s}_{0}}(F, \partial F) \amalg B \operatorname{Diff}^{\mathfrak{s}_{1}}(F, \partial F) .
$$

Since the group $\operatorname{Diff}^{+}(F, \partial F)$ has contractible components [3] the same is true for $\operatorname{Diff}^{\mathfrak{s}_{i}}(F, \partial F)$, and so $B \operatorname{Diff}^{\mathfrak{s}_{i}}\left(\Sigma_{g, 1}, \partial \Sigma_{g, 1}\right) \simeq B \Gamma_{g, 1}^{\mathfrak{s}_{i}}$. Thus the monoid is

$$
\mathcal{M}=\coprod_{g \geq 0} \coprod_{i=0,1} B \Gamma_{g, 1}^{\mathfrak{s}_{i}}
$$

The components of this monoid are $\mathbb{N} \times \mathbb{Z} / 2 \mathbb{Z}$, so it can be group-completed by inverting just $(1,0)$ : this corresponds to gluing on a torus with a spin structure of Arf invariant 0 . The telescope $\mathcal{M}_{\infty}$ is then $\mathbb{Z} \times \mathbb{Z} / 2 \mathbb{Z} \times B \Gamma_{\infty, 1}^{\sigma}$.

If $\theta: \mathbf{X} \rightarrow B O(2)$ is a tangential structure with $\mathbf{X}$ connected and such that $S^{2}$ admits a $\theta$-structure, and $Y$ is a path connected space, then $\theta \times Y=\theta \circ \pi_{\mathbf{X}}: \mathbf{X} \times Y \rightarrow B O(2)$ again has these properties. This allows us to add maps to a background space $Y$ to any such tangential structure.

Let us develop this in the case of ordinary orientations, where the tangential structure is $\theta: B \mathrm{SO}(2) \rightarrow B O(2)$. The space $\mathcal{S}_{g, 1}(Y ; *)$ of Cohen-Madsen [2] is precisely the space $B \operatorname{Diff}^{\theta \times Y}\left(F_{g, 1}, \partial F_{g, 1}\right)$, where the map to $Y$ is fixed to be the constant map to the basepoint on the boundary. Cobordism classes of objects in $\mathcal{C}_{\theta \times Y}^{\bullet}$ are in natural bijection with $H_{1}(Y, \mathbb{Z})$, via the map that sends a circle in $Y$ to the homology class it represents. Let $C$ be a representative of the trivial homology class.

The monoid of endomorphisms of $C$ in $\mathcal{C}_{\theta \times Y}^{\bullet}$ is $\mathcal{M}=\bigsqcup_{g \geq 0} \mathcal{S}_{g, 1}(Y ; *)$. For a general space $Y$ this monoid has tremendously many components, and so it is not clear that one can form the stabilisation $\mathcal{M}_{\infty}$, as one may not be able to group complete $\pi_{0}(\mathcal{M})$ by inverting finitely many elements. However, in several interesting one can form $\mathcal{M}_{\infty}$.

Note that there is a homotopy fiber sequence

$$
\Omega^{2} Y \rightarrow \operatorname{map}\left(\left(F_{g, 1}, \partial F_{g, 1}\right),(Y, *)\right) \rightarrow(\Omega Y)^{2 g}
$$

onto those components of $(\Omega Y)^{2 g}$ represented by tuples $\left(a_{1}, b_{1}, \ldots, a_{g}, b_{g}\right) \in \pi_{1}(Y)^{2 g}$ such that $\prod_{i=1}^{g}\left[a_{i}, b_{i}\right]=1$.

\section{1 $Y$ simply connected}

In this case $\pi_{0}\left(\mathcal{S}_{g, 1}(Y ; *)\right) \cong \pi_{2}(Y) \cong H_{2}(Y ; \mathbb{Z})$, and $\pi_{0}(\mathcal{M})=\mathbb{N} \times H_{2}(Y ; \mathbb{Z})$ as a monoid. This can be group completed by inverting just $(1,0) \in \mathbb{N} \times H_{2}(Y ; \mathbb{Z})$, and 
so we can form $\mathcal{M}_{\infty}$ as the mapping telescope of

$$
\mathcal{M} \stackrel{\cdot(1,0)}{\longrightarrow} \mathcal{M} \stackrel{\cdot(1,0)}{\longrightarrow} \mathcal{M} \stackrel{\cdot(1,0)}{\longrightarrow} \cdots
$$

The discussion in Section 1.1 and Theorem B gives:

Corollary F (Cohen-Madsen [2]) Let $Y$ be a simply connected space. Then there is a homology equivalence

$$
\mathbb{Z} \times \underset{g \rightarrow \infty}{\operatorname{hocolim}} \mathcal{S}_{g, 1}(Y ; *) \rightarrow \Omega^{\infty} \mathbf{M T S O}(2) \wedge Y_{+}
$$

where the colimit is formed by the map $\cdot(1,0)$ above.

\section{2 $Y=B G, G$ a cyclic group}

In this case

$$
\pi_{0}\left(\mathcal{S}_{g, 1}(B G ; *)\right)=\pi_{0}\left(\operatorname{Bun}^{\partial}\left(T F_{g, 1}, \theta^{*} \gamma\right)\right) \times_{\operatorname{Diff}\left(F_{g, 1}\right)} \pi_{0}\left(\operatorname{map}^{\partial}\left(F_{g, 1}, B G\right)\right)
$$

and the homotopy fiber sequence (5-1) gives that

$$
\operatorname{map}^{\partial}\left(F_{g, 1}, B G\right) \simeq G^{2 g} \simeq H^{1}\left(F_{g, 1}, \partial F_{g, 1} ; G\right)
$$

therefore

$$
\pi_{0}(\mathcal{M})=\coprod_{g \geq 0} G^{2 g} / \operatorname{Diff}^{+}\left(F_{g, 1}\right)
$$

where composition is by concatenating (representatives of equivalence classes of) tuples of elements of $G$. Note that this monoid is generated by the $g=1$ elements. This much holds for any abelian group $G$.

Lemma 5.1 Suppose that $G$ is a cyclic group generated by an element 1 . Then in $G^{2} / \operatorname{Diff}^{+}\left(F_{1,1}\right)$ there are relations

$$
[a \pm b, b]=[a, b]=[a, b \pm a] .
$$

In $G^{4} / \operatorname{Diff}^{+}\left(F_{2,1}\right)$ there are relations

and

$$
\begin{aligned}
& {[0,1] \cdot[a, b]=[0,1] \cdot[a-1, b]} \\
& {[0,1] \cdot[0, b]=[0,1] \cdot[0,1]}
\end{aligned}
$$

in the monoid $\pi_{0}(\mathcal{M})$. 
Proof $\operatorname{Diff}^{+}\left(F_{1,1}\right)$ acts on $G^{2}$ through the group $S p(2, \mathbb{Z})$, to which it surjects. The obvious group elements give the relations claimed. $\operatorname{Diff}^{+}\left(F_{2,1}\right)$ acts on $G^{4}$ through the group $\operatorname{Sp}(4, \mathbb{Z})$, to which it surjects. Define an element

$$
t=\left(\begin{array}{cccc}
1 & 1 & 0 & -1 \\
0 & 1 & 0 & 0 \\
0 & -1 & 1 & 1 \\
0 & 0 & 0 & 1
\end{array}\right)
$$

in this group. Then

$$
t(0,1, a, b)=(1-b, 1, a-1+b, b) \sim(0,1, a-1, b) .
$$

We also have

$$
t(0,1,0, b)=(1-b, 1, b-1, b) \sim(0,1, b-1,1) \sim(0,1,0,1) .
$$

Thus inverting $[0,1]$ gives $[a, b]=[0, b]$ in the group-completion, and implies $[0, b]$ is invertible. Thus it is enough to just invert $[0,1]$ to group-complete the monoid, so we may form

$$
\mathcal{M}_{\infty}=\operatorname{hocolim}(\mathcal{M} \stackrel{\cdot[0,1]}{\longrightarrow} \mathcal{M} \stackrel{\cdot[0,1]}{\longrightarrow} \mathcal{M} \stackrel{\cdot[0,1]}{\longrightarrow} \cdots)
$$

whereby Theorem B gives:

Corollary G Let $G$ be a cyclic group. Then there is a homology equivalence

$$
\mathbb{Z} \times \underset{g \rightarrow \infty}{\operatorname{hocolim}} \mathcal{S}_{g, 1}(B G ; *) \rightarrow \Omega^{\infty} \operatorname{MTSO}(2) \wedge B G_{+}
$$

where the colimit is formed by the map $\cdot[0,1]$ above.

Let us spell this result out in certain cases. Recall that $\operatorname{Diff}^{+}\left(F_{g, 1}\right) \simeq \pi_{0}\left(\operatorname{Diff}^{+}\left(F_{g, 1}\right)\right)$ which is the mapping class group $\Gamma_{g, 1}$ of $F_{g, 1}$. When $G=\mathbb{Z}$, we have $\Gamma_{g, 1}$ acting on $\mathbb{Z}^{2 g}$, and let us write $\Gamma_{g, 1}^{\prime}$ for the subgroup of $\pi_{0}\left(\operatorname{Diff}^{+}\left(F_{g, 1}\right)\right)$ which fixes the primitive element $(0,1,0,1, \ldots, 0,1) \in \mathbb{Z}^{2 g}$. The statement of the corollary is then that

$$
\mathbb{Z} \times \operatorname{hocolim}\left(\cdots \rightarrow B \Gamma_{g, 1}^{\prime} \stackrel{\cdot[0,1]}{\longrightarrow} B \Gamma_{g+1,1}^{\prime} \rightarrow \cdots\right) \rightarrow \Omega^{\infty} \mathbf{M T S O}(2) \wedge S_{+}^{1}
$$

is a homology equivalence. This identifies the stable homology of the subgroup $\Gamma_{g, 1}^{\prime}<\Gamma_{g, 1}$ which fixes a primitive element of $H^{1}\left(F_{g, 1}, \partial F_{g, 1} ; \mathbb{Z}\right)$.

When $G=\mathbb{Z} / n \mathbb{Z}$, we have $\Gamma_{g, 1}$ acting on $(\mathbb{Z} / n \mathbb{Z})^{2 g}$, and let us write $\Gamma_{g, 1}^{\prime}(n)$ for the subgroup of $\pi_{0}\left(\operatorname{Diff}^{+}\left(F_{g, 1}\right)\right)$ that fixes the primitive element $(0,1,0,1, \ldots, 0,1) \in$ 
$(\mathbb{Z} / n \mathbb{Z})^{2 g}$. The statement of the corollary is then that $\mathbb{Z} \times \operatorname{hocolim}\left(\cdots \rightarrow B \Gamma_{g, 1}^{\prime}(n) \stackrel{\cdot[0,1]}{\longrightarrow} B \Gamma_{g+1,1}^{\prime}(n) \rightarrow \cdots\right) \rightarrow \Omega^{\infty} \operatorname{MTSO}(2) \wedge B \mathbb{Z} / n \mathbb{Z}_{+}$ is a homology equivalence. On the other hand, $B \Gamma_{g, 1}^{\prime}(n)$ can be identified with the moduli space of Riemann surfaces of genus $g$ with a single framed point, equipped with an $n$-fold cyclic unbranched cover. This identifies the stable homology of these moduli spaces.

A similar analysis holds for any space $Y$ with cyclic fundamental group. The question of whether $\pi_{0}(\mathcal{M})$ may be group completed by inverting finitely many elements for general groups $G=\pi_{1}(Y)$ seems to be difficult.

\section{References}

[1] T Bauer, An infinite loop space structure on the nerve of spin bordism categories, Q. J. Math. 55 (2004) 117-133 MR2068314

[2] R L Cohen, I Madsen, Surfaces in a background space and the homology of mapping class groups, from: "Algebraic geometry-Seattle 2005. Part 1", (D Abramovich, A Bertram, L Katzarkov, R Pandharipande, M Thaddeus, editors), Proc. Sympos. Pure Math. 80, Amer. Math. Soc. (2009) 43-76 MR2483932

[3] C J Earle, J Eells, A fibre bundle description of Teichmüller theory, J. Differential Geometry 3 (1969) 19-43 MR0276999

[4] J Ebert, A vanishing theorem for characteristic classes of odd-dimensional manifold bundles arXiv:0902.4719

[5] S Galatius, Stable homology of automorphism groups of free groups, to appear in Ann. of Math. (2) arXiv:math/0610216

[6] S Galatius, U Madsen, Ib Tillmann, M Weiss, The homotopy type of the cobordism category, Acta Math. 202 (2009) 195-239 MR2506750

[7] J L Harer, Stability of the homology of the mapping class groups of orientable surfaces, Ann. of Math. (2) 121 (1985) 215-249 MR786348

[8] J L Harer, Stability of the homology of the moduli spaces of Riemann surfaces with spin structure, Math. Ann. 287 (1990) 323-334 MR1054572

[9] N V Ivanov, On the homology stability for Teichmüller modular groups: closed surfaces and twisted coefficients, from: "Mapping class groups and moduli spaces of Riemann surfaces (Göttingen, 1991/Seattle, WA, 1991)", (C-F Bödigheimer, R M Hain, editors), Contemp. Math. 150, Amer. Math. Soc. (1993) 149-194 MR1234264

[10] I Madsen, M Weiss, The stable moduli space of Riemann surfaces: Mumford's conjecture, Ann. of Math. (2) 165 (2007) 843-941 MR2335797 
[11] D McDuff, G Segal, Homology fibrations and the "group-completion" theorem, Invent. Math. 31 (1975/76) 279-284 MR0402733

[12] E Y Miller, The homology of the mapping class group, J. Differential Geom. 24 (1986) 1-14 MR857372

[13] G Segal, Categories and cohomology theories, Topology 13 (1974) 293-312 MR0353298

[14] U Tillmann, On the homotopy of the stable mapping class group, Invent. Math. 130 (1997) 257-275 MR1474157

[15] N Wahl, Homological stability for the mapping class groups of non-orientable surfaces, Invent. Math. 171 (2008) 389-424 MR2367024

Department of Mathematics, Stanford University

Stanford CA, 94305, United States

Mathematical Institute

24-29 St Giles', Oxford, OX1 3LB, United Kingdom

galatius@stanford.edu, randal-w@maths.ox.ac.uk

Proposed: Haynes Miller

Received: 29 July 2009

Seconded: Jesper Grodal, Bill Dwyer

Accepted: 19 April 2010 